\title{
HARMONIC ANALYSIS FOR FUNCTORS ON CATEGORIES OF BANACH SPACES OF DISTRIBUTIONS
}

\author{
BY \\ THOMAS DONALDSON
}

\begin{abstract}
This paper develops a theory of harmonic analysis (Fourier series, approximation, convolution, and singular integrals) for a general class of Banach function or distribution spaces. Continuity of singular convolution operators and convergence of trigonometric series is shown with respect to the norms of all the spaces in this class; the maximal class supporting a theory of the type developed in this paper is characterized (for other classes other theories exist). Theorems are formulated in category language throughout. However only elementary category theory is needed, and for most results the notions of functor and natural mapping are sufficient.
\end{abstract}

Introduction. Over the past thirty years the application of Banach space theory to approximation theory, to integral equations, and to differential equations has led to efforts to obtain results on approximation, stability, and existence of solutions in as wide a class of Banach spaces as possible. By now results for most questions of approximation and existence of solutions have been proved for the $L^{p}$ spaces and spaces constructed from them; in some cases results have been extended to the classes of Orlicz spaces or Lorentz spaces; and for linear ordinary differential equations a theory of stability has been worked out as far as general classes of Banach function spaces.

In these applications there are very many cases of Banach spaces all constructed from other Banach spaces according to very similar schemes: a Banach space suited to a given problem will be constructed from other Banach spaces according to a pattern which depends on the problem. As examples one may give the Sobolev spaces or the Lipschitz spaces in partial differential equations; in ordinary differential equations one may adduce the constructions of full or lean spaces in the work of Massera and Shaeffer.

For very many of these results on differential equations or approximation theory, the continuity of integral operators from one space to another plays a crucial part. Very much effort has been devoted to proving such results on continuity for many classes of operators and spaces, such as the $L^{p}$ spaces, the Orlicz spaces, Lorentz spaces, and generalizations of these.

Received by the editors February 23, 1972 and, in revised form, July 18, 1972.

AMS (MOS) subject classifications (1970). Primary 46E30, 42-00, 46M15, 43A95; Secondary 42A18, 42A20, 42A40, 46E35, 43A75.

Key words and phrases. General Banach function space, $L^{p}$ space, Orlicz space, Lorentz space, O'Neil space, Sobolev space, Lipschitz spaces, Fourier series, singular integrals, approximation, inverse theorems, multipliers, Calderón-Zygmund operators, interpolation functors, Hölder functor, Banach space categories.

Copyright $\odot$ 1974, American Mathematical Society 
As a result of this work there has been a very considerable proliferation of Banach spaces constructed in similar ways to fit the study of particular concrete problems.

However, from each of these constructions of Banach spaces from other Banach spaces, one may define a functor on a category of Banach spaces; more important, if $F$ is a functor on a category $B$ of Banach spaces then the natural mappings $\pi: F \rightarrow F$ have a "universal" property. If $\pi: F \rightarrow F$ is a natural mapping, then $\pi: F(X) \rightarrow F(X)$ for every Banach space $X \in \mathcal{B}$. Therefore by showing that an integral operator defines a natural mapping one can obtain, simultaneously, continuity results for integral operators between very wide classes of Banach function spaces: $\pi: F(X) \rightarrow F(X)$ for every Banach space of the form $F(X)$ for $X \in \mathcal{B}$. The proofs of these estimates use the properties of the functor and depend only very weakly on any properties of the underlying space.

In this paper I have commenced a detailed study of the natural mappings between some (and only some) of the functors of applied analysis: specifically those functors which arise from the application of translation operators to the elements of the Banach space. Whether a mapping is natural depends both upon the functor and upon the category on which it acts; the largest class of spaces which seems to support a reasonable theory for functors derived from translation is the class of harmonically invariant Banach spaces, which may be characterized briefly as that class of Banach spaces closed under the applications of the operations of harmonic analysis. For some important functors on the category of harmonically invariant spaces surprisingly strong theorems on natural mappings may be proven.

It seems to me that the results of this paper have the potential of unifying many of the results obtained in applications using the functors studied here or derived from them; in particular they suggest the program of developing approximation theory and differential equations in terms of functors on categories of Banach spaces rather than for individual Banach spaces themselves.

The role of category theory in these results is that of providing a framework within which the main results may be formulated; the crucial ideas are those of functor and natural mapping. No very difficult results of category theory seem to be required. In most cases the action of the functors on morphisms in the categories studied does not play an important role; however I have dealt explicitly with the actions of morphisms and commutativity of diagrams in preference to ignoring them completely.

This paper constitutes a longer study of the implications of Theorem 1.1 in the announcement [7]. The basic technique is very simple; I shall illustrate with a particular case of the result in [7]. The Lipschitz spaces $C^{r}$ on $[0,2 \pi]$ for $0<r<1$ are quite well known in analysis. It is known for instance that the Hilbert transform $H$ is continuous from $C^{r}$ to $C^{r}$. Let $X$ be a Banach space and

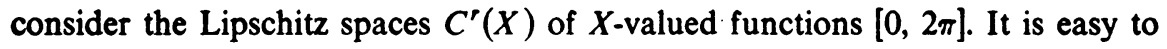
show that $H: C^{r}(X) \rightarrow C^{r}(X)$ for any Banach space $X$. 
Now suppose that $X$ is a Banach space of integrable functions on $[0,2 \pi]$, and that the norm of $X$ is continuous and invariant with respect to translation, i.e. for every $f \in X, \tau(f)(s)=T_{s} f$ is a continuous $X$-valued function of $s$, and $T_{s}$ is an isometry.

Combine these two facts. Let $\Lambda C^{r}(X)$ be the class of all $f \in X$ such that the $X$-valued function $\tau(f) \in C^{r}(X)$, and norm $\Lambda C^{r}(X)$ with the induced norm. The Hilbert transform therefore maps $\tau(f) \in C^{r}(X)$ continuously into $C^{r}(X)$, and one has the inequality

$$
\|H(\tau f)\|_{C^{\prime}(X)} \leq k\|\tau f\|_{C^{\prime}(X)}=k\|f\|_{\Lambda C^{\prime}(X)} .
$$

But $H(\tau f)(s)=\int h(s-t) T_{t} f(x) d t=\int h(s-t) f(x+t) d t=T_{s} \int h(x-t) f(t) d t$ $=\tau(H f)(s)$ so that, therefore, from the inequality (0.1) it follows that $\|H f\|_{\Lambda C^{r}(X)}$ $\leq k\|f\|_{\Lambda C^{\prime}(X)}$ and $H: \Lambda C^{r}(X) \rightarrow \Lambda C^{\prime}(X)$ for any space $X$ with the properties above.

This technique has wider application: it may be extended for instance to Banach spaces of distributions; the requirement of continuity of the norm of $X$ under translation may be removed; and the same technique may be applied to other functors and problems. Finally since no part of the method is restricted in any natural way to the cases of either spaces or convolution kernels with only complex values, and since moreover there are important applications of vectorvalued spaces and kernels, I have developed the theory throughout from that standpoint.

I thank Richard Loy for referring me to the references cited on Banach modules and tensor products of Banach modules, and Robert Edwards for calling my attention, while this paper was in preparation, to the book of Katznelson [15] in which weaker results are proved for a more special class of spaces.

\section{CHAPTER 1: PRELIMINARIES}

In this paper I shall deal with Banach spaces and Banach modules. The notion of Banach space is familiar; a Banach module is a Banach space $B$ together with a Banach algebra $R$ of operators acting on $B$. Every Banach space is a Banach module over the ring $\mathbf{R}$ or $\mathbf{C}$ of the underlying field. Several authors (Rieffel [30], [31], Comisky [5], and in his book [11], Edwards alludes to the concept in passing) have already introduced the notion of Banach module into harmonic analysis, where the idea of such a structure arises naturally.

From the class of Banach spaces one may construct a corresponding category $\mathcal{B}$, the category of Banach spaces. For $X, Y \in \mathcal{B}$, the mappings $[X, Y]$ consist of the continuous linear mappings from the Banach space $X$ to the Banach space $Y$. The category $\mathcal{B}$ will be of much importance in the sequel, and $\mathcal{B}$ also has important subcategories. 
Let $T^{n}$ be a toroidal group and $E=\left\{e_{m} \mid m \in Z^{n}\right\}$ the characters of $T^{n}$. For every Banach space $X$ of complex-valued distributions on $T^{n}$, let $E_{X}=$ $\left\{e_{m} \in E \mid \exists \lambda \in X \ni \lambda(m) \neq 0\right\}$, and $C_{X}^{\infty}=\left\{\varphi \in C^{\infty} \mid \hat{\varphi}(m)=0 \forall m \ni e_{m}\right.$ $\left.\notin E_{X}\right\}$. I shall say that a Banach space $X$ of distributions on $T^{n}$ is harmonically invariant if

(1) $\left\|T_{s} \lambda\right\|_{X}=\|\lambda\|_{X}$ for every translation $T_{s}$ with $s \in T^{n}$,

(2) $C_{X}^{\infty}$ is a norming subspace of $X^{*}$,

(3) $E_{X} \subseteq X$.

The category $\mathcal{B}_{00}$ will then consist of all harmonically invariant Banach spaces of complex-valued distributions on $T^{n}$. The mappings $[X, Y]$ for $\mathcal{B}_{00}$ will consist of the continuous linear mappings from $X$ to $Y$, and $\mathcal{B}_{00}$ is clearly a subcategory of $\mathcal{B}$. The category $\mathcal{B}_{00}$ includes very many Banach spaces which have arisen in analysis, such as the Lorentz spaces $\Lambda(\phi, p), M(\phi, p), \Gamma(\phi, p)$ [19], [20], the Orlicz spaces $L_{B}$ studied by very many authors [17], [26], the spaces $M_{A}$ and $K_{A}$ studied by O'Neill [26], or the Köthe spaces [8].

The category $\mathcal{B}_{\omega}$ forms also a second important subcategory of $\mathcal{B}$. Let $T^{n}$ be a toroidal group and define $\mathcal{B}_{\omega}$ as the category of all Banach spaces of complexvalued distributions on $T^{n}$; let the linear mappings for $\mathcal{B}_{\omega}$ consist simply of the identity mapping whenever it is continuous from $X \in \mathcal{B}_{\omega}$ to $Y \in \mathcal{B}_{\omega}$.

Classes of Banach modules will also form categories. Let $L^{\mathbf{l}}$ be the algebra of integrable functions on $T^{n}$, and $H_{0}$ the algebra of all point measures $\delta_{s}$ for $s \in T^{n}$; let $M_{0}=L^{1} \oplus H_{0}$. From the class of all Banach modules over $M_{0}$, one may construct the category $M$. The mappings for $M$ will be the class of continuous module homomorphisms: the class of all those mappings $\alpha: X \rightarrow Y$ for $X, Y \in \mathcal{O}$ which commute with convolution by $h \in M_{0}$.

on also has important subcategories. Let $X$ be a Banach module over $M_{0}$; define for every $\lambda \in X$,

$$
\|\lambda\|_{X}^{\prime}=\sup _{\|h\|_{h} \leq 1}\|h * \lambda\|_{X} \quad \text { where } h \in L^{1} .
$$

If the norm $\|\cdot\|_{X}^{\prime}$ is equivalent to the norm $\|\cdot\|_{X}$ for $X$, I shall say that the module $X$ is normal. The category $M_{00}$ is the category of all normal Banach modules over $M_{0}$, consisting of complex-valued distributions on $T^{n}$. Very many Banach spaces of analysis may be seen as normal Banach modules over $M_{0}$; if every $\lambda \in X$ may be approximated by an approximate identity $\varphi_{k} \in L$, it is clear that $X$ is normal. As a consequence of Theorem 4.0 below, indeed any harmonically invariant Banach space will also be a Banach module over $M_{0}$; and thus as classes of spaces $M_{00}$ and $B_{00}$ coincide, though not as categories.

1. Functors and natural mappings. The concepts of functor and natural mapping will be important to the treatment of harmonic analysis in this paper.

Definition 1.0. Let $C$ and $D$ be categories of Banach modules and $F$ a function $F: C \rightarrow D$ such that $F(X) \in D$ for every $X \in C$ and $F(\alpha) \in[F(X), F(Y)]$ for every $\alpha \in[X, Y]$. $F$ will be termed a covariant functor on $C$ if: 
(1) If $\alpha: X \rightarrow Y, \beta: Y \rightarrow Z$, then $F(\beta \circ \alpha)=F(\beta) \circ F(\alpha)$.

(2) $F\left(l_{X}\right)=1_{F(X)} \forall X \in C$.

(3) The mapping $F:[X, Y] \rightarrow[F(X), F(Y)]$ is of norm $\leq 1$.

A reader familiar with category theory should note condition (3) involves a deviation from the algebraic definition of functor.

I shall refer to a function $F: C \times C \rightarrow C$ which is a functor in each variable separately as a bifunctor.

Several functors in general categories of Banach modules will appear frequently. Most of the following paper will be taken up with a discussion of the properties of certain concrete functors; other functors will play a very important technical role. In this section I shall give a brief discussion of the fundamental properties of functors of these two types.

Definition 1.1. Let $C$ be a category of Banach modules over a commutative ring $R$, and suppose that ring multiplication commutes with the elements of $X$ for every $X \in C$. Let $X, Y \in C$ and define $\Phi^{R}$ to be the module over $R$ of functions on $X \times Y$ to $R$ that differ from 0 only on a finite set of points

$$
\Phi^{R}=\left\{\sum^{N} r_{k}\left\{x_{k}, y_{k}\right\} \mid x_{k} \in X, y_{k} \in Y, r_{k} \in R\right\}
$$

Let $\Phi_{0}^{R}$ be the subspace of $\Phi^{R}$ consisting of elements of the form

$$
\Phi_{0}^{R}=\left\{\sum^{N} r_{k} x_{k}, \sum^{M} r_{j}^{\prime} y_{j}\right\}-\sum_{k=1}^{N} \sum_{j=1}^{M} r_{k} r_{k}^{\prime}\left\{x_{k}, y_{j}\right\} .
$$

Define the algebraic tensor product of the modules $X$ and $Y$ by $X \otimes_{R}^{a} Y$ $=\Phi^{R} / \Phi_{0}^{R} . \Phi^{R} / \Phi_{0}^{R}$ is well defined since $\Phi^{R}$ is clearly Abelian under + . The image $\{x, y\}+\Phi_{0}^{R}$ under the quotient map $\Phi^{R} \rightarrow \Phi^{R} / \Phi_{0}^{R}$ may be denoted by $x \otimes_{R} y$. The module $X \otimes_{R}^{a} Y$ may be given a norm by

$$
\|u\|_{X \otimes_{R} Y}=\inf \left\{\sum_{1}^{N}\left\|r_{k}\right\|_{R}\left\|x_{k}\right\|_{X}\left\|y_{k}\right\|_{Y} \mid u=\sum^{N} r_{k} x_{k} \otimes y_{k}\right\}
$$

Clearly $\left\|x \otimes_{R} y\right\|_{X \otimes_{R} Y}=\|x\|_{X}\|y\|_{Y}$.

I will denote the completion of $X \otimes_{R}^{a} Y$ under the norm (1.2) $\|\cdot\|_{X \otimes_{R} Y}$ by $X \otimes_{R} Y$. Clearly $X \otimes_{R} Y$ is a Banach module over $R$, and therefore is in $C$.

The action of the functor $\otimes_{R}$ on morphisms $\alpha \in\left[X, X_{1}\right], \beta \in\left[Y, Y_{1}\right]$ is given as follows: the operator $\alpha \otimes_{R} \beta$ is defined by

$$
\alpha \otimes_{R} \beta(x \otimes y)=(\alpha x) \otimes_{R}(\beta y) \text { for } x \in X, y \in Y .
$$

It is clear that by the definition (1.2) of the norm $\|\cdot\|_{X \otimes_{R} Y}$,

$$
\left\|\alpha \otimes_{R} \beta\right\|_{\left(X \otimes_{R} Y, X_{1} \otimes_{R} Y_{1}\right\}} \leq\|\alpha\|_{\left\{X, X_{1}\right\}}\|\beta\|_{\left\{Y, Y_{1}\right\}}
$$


I shall denote a tensor product $X \otimes_{R} Y$ over the real or complex fields simply by $X \otimes Y$.

The function from $C \times C \rightarrow C$ defined by Definition 1.1 is a function of two variables, which may easily be seen to satisfy Definition 1.0 in either variable separately.

It should be emphasized that the definition of norm in (1.2) is not unique. Schatten [33] has developed the definitions and properties of several tensor products of Banach spaces under the name of "cross norms". The analogous development for arbitrary Banach modules has been commenced by Rieffel [30]. Tensor products for general locally convex topological vector spaces were studied by Grothendieck [13]. If the ring $R$ in (1.2) is the ring of real or complex numbers, the norm in (1.2) will correspond to the unique greatest cross norm in the sense of Schatten and the projective tensor product in the sense of Grothendieck.

The functor $\otimes_{R}$ gives rise to a second functor important for the general category theory of Banach modules.

Definition 1.2. Let $A$ be a fixed Banach module and let $C$ be the category of Banach modules over the ring $R$. For every $X \in C$, one may define a functor $\Sigma_{A}$ by

$$
\begin{aligned}
& \Sigma_{A}(X)=A \otimes_{R} X, \\
& \Sigma_{A}(\alpha)=1_{A} \otimes_{R} \alpha \text { for } \alpha \in[X, Y] .
\end{aligned}
$$

It is immediate that $\Sigma_{A}$ is a well-defined functor on $C$.

A second type of functor may also be defined in any category of Banach modules.

Definition 1.3. Let $C$ be a category of Banach modules over $R$, and let $A \in C$. Then one may define the functor $\Omega_{A}: C \rightarrow C$ by

$$
\begin{array}{ll}
\Omega_{A}(X)=[A, X] & \text { for every } X \in C, \\
\Omega_{A}(\alpha)=\alpha \circ \lambda & \text { for every } \lambda \in[A, X] \text { and } \alpha \in[X, Y] .
\end{array}
$$

Clearly $\Omega_{A}$ will also define a functor. From Definitions 1.7 and 1.8 below it will be clear that some very important functors are of the form defined by (1.3).

Some other types of functors important for technical purposes are given by the following definitions:

Definition 1.4. Let $C$ be a category of Banach modules and $F$ a functor $F: C \rightarrow C$. Then

(1) If $G$ is a functor on $C$ such that

(i) for every $X \in C, G(X)$ is a closed submodule of $F(X)$,

(ii) $G(\alpha): F(\alpha): G(X) \rightarrow G(Y)$ for every $\alpha \in[X, Y]$,

then the functor $G$ will be termed a closed subfunctor of $F$.

(2) If $G$ is a functor on $C$ such that 
(i) for every $X \in C, G(X) \subseteq F(X)$ and the identity mapping $I: G \rightarrow F$ is natural,

(ii) $G(\alpha)=F(\alpha): G(X) \rightarrow G(Y)$ for every $\alpha \in[X, Y]$, then the functor $G$ will be termed a norm subfunctor of $F$.

Definition 1.5. Let $C$ be a category of Banach modules, $F$ a functor on $C$, and $G$ a subfunctor of $F$. Then the quotient functor $H=F / G$ of $F$ and $G$ is defined by

$$
\begin{aligned}
H(X) & =F(X) / G(X) \text { for every } X \in C, \\
H(\alpha)(f+G(X)) & =F(\alpha)(f)+G(Y) .
\end{aligned}
$$

Since $G(\alpha)=F(\alpha): G(X) \rightarrow G(Y)$ for every $\alpha \in[X, Y],(1.4)$ will define a unique functor on the category $C$.

Examples of each of these types will be given below.

The $C^{\infty}$ functor and the distribution functor will also play an important technical role in definitions and proofs.

Definition 1.6. Let $T^{n}$ be a toroidal group and $\mathcal{B}$ the category of Banach spaces.

(I) For every $X \in \mathcal{B}$, define the $C^{\infty}$ functor by

$$
C^{\infty}(X)=\left\{\varphi: T^{n} \rightarrow X \mid \varphi \text { infinitely differentiable }\right\} .
$$

The topology on $C^{\infty}(X)$ is defined by the class of seminorms

$$
\begin{gathered}
P^{m}(\varphi)=\sup _{s \in T^{n}}\left\|D^{m} \varphi\right\|_{X}, \\
C^{\infty}(\alpha)(\varphi)(t)=\alpha \varphi(t) \quad \forall t \in T^{n} .
\end{gathered}
$$
by

(II) Let $C^{\infty}=C^{\infty}(\mathbf{C})$ and define the distribution functor $D$ for every $X \in \mathcal{B}$

$$
\begin{aligned}
D(X) & =\left\{\lambda: C^{\infty} \stackrel{\text { cont. }}{\rightarrow} X\right\}, \\
D(\alpha)(\lambda) & =\alpha \circ \lambda \quad \text { for every } \alpha \in[X, Y], \lambda \in D(X) .
\end{aligned}
$$

Clearly neither the distribution functor $D$ nor the $C^{\infty}$ functor are Banach functors since $D(X)$ is not a Banach space. The study of this functor was initiated by L. Schwartz [36].

The body of this paper will be concerned with four important concrete functors (and their subfunctors) acting from the category $\mathcal{B}$ of Banach spaces to the category $M$ of Banach modules over $M_{0}$.

Definition 1.7. Let $T^{n}$ be a toroidal group, $\mathcal{B}$ be the category of Banach spaces, and $C$ and $L^{1}$ the Banach spaces of continuous and integrable functions on $T^{n}$ respectively. Denote the functor $\Omega_{C}$ by $M$ and the functor $\Omega_{L^{\prime}}$ by $L^{\infty}$. Then $L^{\infty}: \mathcal{B} \rightarrow \mathcal{C}$ and $M^{*}: \mathcal{B} \rightarrow \mathcal{M}$ where the ring multiplication is defined for every $X \in \mathcal{B}$ by 


$$
\begin{array}{ll}
\mu * \lambda(\varphi)=\lambda(\mu * \varphi) & \text { for every } \lambda \in M^{*}(X)=[C, X], \\
\mu * \lambda(\varphi)=\lambda(\mu * \varphi) & \text { for every } \lambda \in L^{\infty}(X)=\left[L^{1}, X\right] .
\end{array}
$$

The functors $L^{\infty}$ and $M^{*}$ both have very important subfunctors. The first of these will be of very great importance in Chapter 4 .

Definition 1.8. Let $T^{n}$ be a toroidal group, $\mathcal{B}$ the category of Banach spaces, and $M^{*}=\Omega_{C}$ the measure functor on $\mathcal{B}$. For every $X \in \mathcal{B}$ and $\lambda \in M^{*}(X)$, define a norm

$$
\|\| \lambda\left\|_{M_{0}^{*}(x)}=\sup _{N, \xi_{k}, \varphi_{k}} \sum^{N}\right\| \lambda\left(\varphi_{k}\right) \|
$$

where the sup in (1.5) is taken over all sets of scalars $\left|\xi_{k}\right| \leq 1$ and $\varphi_{k} \in C$ such that $\left\|\Sigma^{N} \xi_{k} \varphi_{k}\right\| \leq 1$.

Define a functor $M: \mathcal{B} \rightarrow \mathcal{M}$ for every $X \in \mathcal{B}$ by

$$
\begin{aligned}
M(X) & =\left\{\lambda \in M^{*}(X) \mid\|\| \lambda \|_{M_{0}^{*}(X)}<\infty\right\}, \\
\|\lambda\|_{M(X)} & =\|\| \lambda \|_{M_{0}^{*}(X)} \\
M(\alpha) \lambda & =M^{*}(\alpha) \lambda=\alpha \circ \lambda .
\end{aligned}
$$

The norm (1.5) is not new; it describes the class of $\lambda \in[C, X]$ which are integral in the sense of Grothendieck [13, Definition 7 and Proposition 32, p. 145].

It is clear that the functor $M$ is a norm subfunctor of the functor $M^{*}$. For most purposes the functor $M$ is easier to deal with than the functor $M^{*}$ and is indeed more natural.

It is also easy to see that $\|\lambda\|_{M^{\bullet}(x)} \leq\|\lambda\|_{M(X)}$ for every $X \in \mathcal{B}$.

A second important type of subfunctor is given by the next definition. Let $T^{n}$ be a toroidal group and denote $T_{s}$ the operator of translation by $s \in T^{n}$ defined by $T_{s} f(t)=f(s+t)$ for every $f \in L^{1}$ and every $s, t \in T^{n}$. It is easy to see that $T_{s}$ may be defined in terms of convolution with a measure $\delta_{s}$, the Dirac delta distribution at $\delta$, and thus $T_{s}$ is defined as an operator on $M(X)$ and $L^{\infty}(X)$ for every $X \in \mathcal{B}$. The operator $T_{s}$ will be termed the translation operator.

Definition 1.9. Let $T^{n}$ be a toroidal group and $\mathcal{B}$ the category of Banach spaces. Then define the functors $C: \mathcal{B} \rightarrow \mathcal{O}$ and $L^{1}: \mathcal{B} \rightarrow \mathcal{O}$ for every $X \in \mathcal{B}$ by

$$
\begin{array}{rlrl}
C(X) & =\left\{\lambda \in L^{\infty}(X) \mid\left\|T_{s} \lambda-\lambda\right\|_{L^{\infty}(X)} \rightarrow 0 \text { as } s \rightarrow 0\right\}, & C(\alpha)=L^{\infty}(\alpha) ; \\
L^{1}(X)=\left\{\lambda \in M^{*}(X) \mid\left\|T_{s} \lambda-\lambda\right\|_{M(X)} \rightarrow 0 \text { as } s \rightarrow 0\right\}, & L^{\prime}(\alpha)=M(\alpha) .
\end{array}
$$

The following proposition follows easily from Definitions 1.4 and 1.9.

Proposition 1.0. Let $B$ be the category of Banach spaces and $L^{\infty}, M$ and $C, L^{1}$ the functors of Definitions 1.8 and 1.9 respectively. Then $C$ is a subfunctor of $L^{\infty}$ and $L^{1}$ 
is a subfunctor of $M$.

Proof. Clearly $C(X)$ is a closed submodule of $L^{\infty}(X)$ and $L^{1}(X)$ is a closed submodule of $M^{*}(X)$ for every $X \in \mathcal{B}$. Furthermore from Definition 1.8 it follows that, for both of the functors $L^{\infty}$ and $M^{*}, \alpha \circ T_{s} \lambda(\varphi)=\alpha \circ \lambda\left(T_{s} \varphi\right)$ $=T_{s}(\alpha \circ \lambda)(\varphi)$, and therefore $\alpha \circ T_{s} \lambda=T_{s}(\alpha \circ \lambda)$ for $\lambda \in L^{\infty}(X)$ or $M(X)$ and every $X \in \mathcal{B}$. By Definition 1.8,

$$
\left\|T_{s}(\alpha \circ \lambda)-\alpha \circ \lambda\right\|=\left\|\alpha\left(T_{s} \lambda-\lambda\right)\right\| \leq\|\alpha\|\left\|T_{s} \lambda-\lambda\right\|
$$

for both functors $L^{\infty}$ and $M^{*}$. Thus for every $X, Y \in \mathcal{B}$ and $\alpha \in[X, Y]$,

$$
\begin{array}{rlrl}
C(\alpha) & =L^{\infty}(\alpha): C(X) \rightarrow C(Y) & \text { where } C(\alpha) \lambda=\alpha \circ \lambda, \\
L^{\prime}(\alpha)=M^{*}(\alpha): L^{1}(X) \rightarrow L^{1}(Y), & L^{\prime}(\alpha) \lambda=\alpha \circ \lambda .
\end{array}
$$

$C$ and $L^{\prime}$ are therefore subfunctors of $L^{\infty}$ and $M^{*}$ respectively.

I shall also deal with a second important pair of functors.

Definition 1.10. Let $T^{n}$ be a toroidal group and $\mathcal{B}$ the category of Banach spaces. For every real number $r, 0<r<1$, define functors $C^{r}$ and $M^{r}$ acting from $B$ to $M$ by

$$
\begin{aligned}
C^{\prime}(X) & =\left\{\lambda \in L^{\infty}(X) \mid \sup _{s \in T^{n}} \frac{\left\|T_{s} \lambda-\lambda\right\|_{L^{\infty}(X)}}{|s|^{r}}<\infty\right\}, \\
\|\lambda\|_{C^{\prime}(X)} & =\|\lambda\|_{L^{\infty}(X)}+\sup _{s \in T^{n}} \frac{\left\|T_{s} \lambda-\lambda\right\|_{L^{\infty}(X)}}{|s|^{r}}, \\
C^{\prime}(\alpha)(\lambda) & =\alpha \circ \lambda \text { for every } \lambda \in C^{r}(X) \text { and } \alpha \in[X, Y], \\
M^{\prime}(X) & =\left\{\lambda \in M(X) \mid \sup _{s \in T^{n}} \frac{\left\|T_{s} \lambda-\lambda\right\|_{M(X)}}{|s|^{r}}<\infty\right\}, \\
\|\lambda\|_{M^{\prime}(X)} & =\|\lambda\|_{M(X)}+\sup _{s \in T^{n}} \frac{\left\|T_{s} \lambda-\lambda\right\|_{M(X)}}{|s|^{r}}, \\
M^{\prime}(\alpha)(\lambda) & =\alpha \circ \lambda .
\end{aligned}
$$

Clearly $C^{r}$ and $M^{\prime}$ are well-defined functors. $C^{\prime}$ has appeared in the form of a functor before; indeed $C^{r}$ plays a very important role in some theories of interpolation (in particular Calderón [3]) where $C^{r}$ and higher order versions of $C^{r}$ yield descriptions of the spaces intermediate between Sobolev spaces $W^{m} L^{p}$ and $W^{l} L^{p}$ for integers $m$ and $l$.

The central concept of this paper is that of a natural mapping of Banach functors. Most theorems will center about a proof that a mapping is natural.

Definition 1.11. Let $C$ be a category of Banach spaces and $F$ and $G$ functors $F: C \rightarrow C$ and $G: C \rightarrow C$. If $T$ is a function which assigns to each object $X \in C$ 
an operator $T_{X}: F(X) \rightarrow G(X)$, then $T$ is termed a natural transformation $T: F \rightarrow G$ of $F$ into $G$ if:

(1) For every $X ; Y \in C$ and $\alpha \in[X, Y]$ the diagram

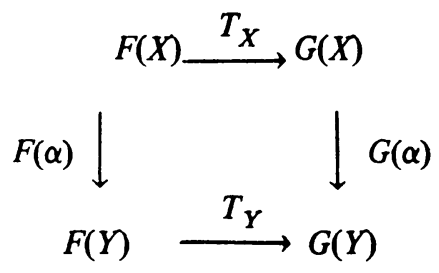

commutes.

(2) The norm of $T_{X}$ as a mapping from $F(X)$ to $G(X)$ is uniformly bounded for all $X \in C$.

If $F$ and $G$ are two functors, the class of natural mappings from $F$ to $G$ will be written $[F, G]$. It is easy to see that $[F, G]$ may itself be given the structure of a Banach space with the norm for $T \in[F, G]$ defined by $\|T\|=\sup _{X}\left\|T_{X}\right\|$.

Definition 1.11 is due to Mityagin and Švarc [24]; the second part of Definition 1.11 becomes a natural requirement for functors acting in categories of Banach spaces. The important observation that the class $[F, G]$ of natural mappings from $F$ to $G$ becomes a Banach space is also due to Mityagin and Svarc.

With the concept of natural mapping of functors it now becomes possible to define the notions of dual functor and tensor multiplier functor. The idea of dual functor plays a similarly commanding role in the category theory of functional analysis to that played by the notion of dual space in ordinary functional analysis; the tensor multiplier functor is the analogue, along the same lines, of the class of convolution multipliers from a Banach module $X$ to $Y$.

Definition 1.12. Let $C$ be a category of Banach spaces and $F: C \rightarrow C$ a functor acting on $C$. A functor $G$ will be referred to as the dual functor to $F$, and written $D F$, if for every $X \in C, G(X)=\left[F, \Sigma_{X}\right]$ and for all mappings $\alpha \in[X, Y]$ and $\gamma \in\left[F, \Sigma_{X}\right]$, and any $Z \in C,[G(\alpha) \gamma]_{X}=\left(1_{X} \otimes \alpha\right) \gamma$.

The idea of dual functors seems to be due to Svarc [40]. For the category $B$ of Banach spaces Mityagin and Švarc [24] have proven that the class of mappings $\left[F, \Sigma_{X}\right]$ may be identified with a subspace of $F\left(X^{*}\right)^{*}$ for every $X \in \mathcal{B}$, and in particular that for any category $C$ of Banach spaces such that $X \in C$ implies $X^{*} \in C$, the functor $D F$ is well defined.

The following proposition about the dual functor will be useful.

Proposition 1.1. Let $F: \mathcal{B} \rightarrow \mathcal{B}$ be a functor acting on the category $\mathcal{B}$ of Banach spaces, and define the mapping $\nu_{X}: D F(X) \rightarrow[F(C) \rightarrow X]$ by $\nu_{X}(\alpha)=\alpha_{C}$ for every $\alpha \in D F(X)=\left[F, \Sigma_{X}\right]$. Then $\nu: D F \rightarrow \Omega_{F(C)}$ is a natural mapping.

The proof of the proposition is immediate from the definition (see Mityagin and Švarc [24, §3.3]). 
I shall now define the tensor multiplier functor.

Let $T^{n}$ be a toroidal group and $M$ the category of Banach modules by convolution over the ring $M_{0}$, consisting of possibly vector-valued Schwartz distributions on $T^{n}$

Definition 1.13. Let $T^{n}$ be a toroidal group, $\mathcal{B}$ the category of Banach spaces, and $F$ and $G$ two covariant functors $F: B \rightarrow \mathcal{B}$ and $G: B \rightarrow \mathcal{B}$. Define a functor $\operatorname{Hom}_{\circledast}[F, G]: \mathcal{B} \rightarrow \mathcal{O}$ by

$$
\begin{aligned}
\operatorname{Hom}_{\otimes}[F, G](X) & =\left[F, G \circ \Sigma_{X}\right], \\
\|h\|_{\mathrm{Hom}_{\odot}[F, G)(X)} & =\|h\|_{\left\{F, G \circ \Sigma_{X}\right]} \text { for every } h \in\left[F, G \circ \Sigma_{X}\right], \\
\operatorname{Hom}_{\otimes}[F, G](\alpha)\left(h_{E}\right) & =G\left(\alpha \otimes 1_{E}\right) h_{E}
\end{aligned}
$$$$
\text { for every } h \in\left[F, G \circ \Sigma_{X}\right] \text { and } E \in \mathcal{B} \text {. }
$$

If $H$ is a functor on $B$ of the form (1.6) for some $F$ and $G, H$ will be termed the tensor multiplier functor corresponding to $F$ and $G$. I should remark also that if $\lambda$ is an $X$-valued distribution and $F$ and $G$ are suitable functors, then $\lambda$ will define an element of $\operatorname{Hom}_{\otimes}[F, G](X)$ by $\lambda * \gamma$ for every $\gamma \in F(Y)$, where * denotes tensor convolution (see Definition 1.21). I shall term a tensor multiplier of such a form a tensor convolution multiplier. The functor $\operatorname{Hom}_{\otimes}[F, G]$ is the analogue for the functors $F$ and $G$ of the Banach spaces $[X, Y]$ of convolution multipliers from the Banach modules $X$ to $Y$.

I shall now prove or state some of the basic theorems on natural transformations for the Banach functors defined above.

The first of these will be two useful propositions on the tensor product $\otimes_{R}$.

Proposition 1.2. Let $C$ be a category of Banach modules over a ring $R$, and suppose that $X, Y, Z \in C$. Then there exists a natural transformation establishing an isometric isomorphism

$$
X \otimes_{R}\left[Y \otimes_{R} Z\right] \cong\left[X \otimes_{R} Y\right] \otimes_{R} Z
$$

Therefore in particular the tensor product may be assumed to be associative.

Proof. Consider first the elements of the algebraic tensor products. Let

$$
\begin{array}{r}
\sum r_{k}\left\{\sum r_{j}^{\prime}\left\{x_{j}^{k}, y_{j}^{k}\right\}+\Psi_{0}, z^{k}\right\}+\Phi_{0} \in\left[X \otimes_{R}^{a} Y\right] \otimes_{R}^{a} Z, \\
\sum q_{k}^{\prime}\left\{x_{k}, \sum q_{j}^{\prime},\left\{y_{j^{\prime}}^{k^{\prime}}, z_{j^{\prime}}^{k^{\prime}}\right\}+\Delta_{0}\right\}+\Xi_{0} \in X \otimes_{R}^{a}\left[Y \otimes_{R}^{a} Z\right]
\end{array}
$$

be general elements of each tensor product. Establish an isomorphism $\tau$ by

$$
\tau\left[\left\{\{x, y\}+\Psi_{0}, z\right\}+\Phi_{0}\right]=\left\{x,\{y, z\}+\Delta_{0}\right\}+\Xi_{0}
$$

and extend $\tau$ by the condition that $\tau$ commutes with ring elements $r \in R$ and the conditions $(1.0)$ for the definition of the subspaces $\Psi_{0}, \Phi_{0}, \Delta_{0}, \Xi_{0}$. Clearly $\tau$ will 
be an algebraic isomorphism. It is also clear by the definition (1.2) of the norms in $X \otimes_{R}\left[Y \otimes_{R} Z\right]$ and $\left[X \otimes_{R} Y\right] \otimes_{R} Z$ that the algebraic isomorphism establishes an isometry of normed modules; this isometry may therefore be extended by completion.

Proposition 1.3. Let $C$ be a category of Banach modules over a ring $R$, and suppose $X, Y, Z \in C$. Then there exists an isometric isomorphism

$$
\left[X \otimes_{R} Y, Z\right] \cong[Y,[X, Z]] . \cong[X,[Y, Z]]
$$

Proof. It will be sufficient to show the first isomorphism, since the second may be established in the same way. Define a mapping $\tau:\left[X \otimes_{R} Y, Z\right] \rightarrow[Y,[X, Z]]$ by

$$
[\tau(u)(y)] x=u\left(x \otimes_{R} y\right) \text { for all } u \in\left[X \otimes_{R} Y, Z\right] \text { and } x, y \in X, Y
$$

and extend by the condition of commutativity of $\tau$ with $r \in R$. It is clear from Definition 1.1 of the norm in $X \otimes_{R} Y$ that

$$
\|u\|_{\left\{X \otimes_{R} Y, Z\right]}=\|\tau(u)\|_{\{,[X, Z)\}} .
$$

Let $C$ be a category of Banach modules over a ring $R$. Proposition 1.3 says that $\left[X \otimes_{R} Y \rightarrow Z\right]$ may be identified with the class of $R$-balanced bilinear mappings $\lambda: X \times Y \rightarrow Z$. In this way $\otimes_{R}$ defines a universal bilinear product from $X \times Y$ to $Z$.

The next proposition, although quite simple, states a third useful property of $\otimes$.

Proposition 1.4. Let $F: \mathcal{B} \rightarrow \mathcal{B}$ be a functor acting on the category $\mathcal{B}$ of Banach spaces, and let $\bar{F}$ denote the Banach space $\bar{F}=F(C)$. Define for every $X \in \mathcal{B}$ the mapping $c_{X}: \bar{F} \otimes X \rightarrow F(X)$ by

$$
i_{X}(f \otimes x)=F(\hat{x}) f \text { where } \hat{x}: \mathbf{C} \rightarrow X \text { is defined by } \hat{x}(1)=x
$$

and $i_{X}$ is extended from (1.7) by linearity and continuity. Then $i: \Sigma_{E} \rightarrow F$ is a natural mapping.

Proof. To see that $i$ is natural, observe simply that the diagram

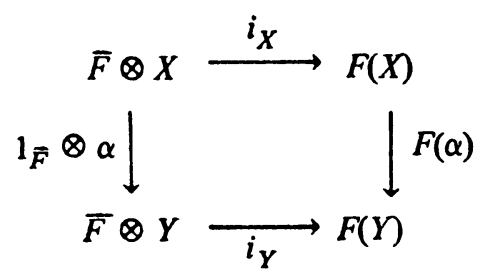

commutes since $F(\alpha) i_{X}(f \otimes x)=i_{Y}(f \otimes \alpha x)$. 
Some very important natural mappings are given by the following representation theorems for the subfunctors $C$ and $L^{l}$ of $L^{\infty}$ and $M^{*}$.

Definition 1.14. Let $T^{n}$ be a toroidal group and $\mathcal{B}$ be the category of Banach spaces. Define the functor $L: \mathcal{B} \rightarrow \mathcal{M}$ for every $X \in \mathcal{B}$ by

$$
\begin{aligned}
L(X) & =\left\{f: T^{n} \rightarrow X \mid f \text { Bochner integrable }\right\}, \\
\|f\|_{L(X)} & =\int\|f(t)\|_{X} d t, \\
L(\alpha)(f)(t) & =\alpha f(t) \quad \forall \alpha \in[X, Y], f \in L(X), t \in T^{n} .
\end{aligned}
$$

$L$ clearly satisfies the requirements for a functor.

It is now possible to state an important and useful representation theorem.

Theorem 1.0. Let $T^{n}$ be a toroidal group and $\mathcal{B}$ be the category of Banach spaces. Let $L$ be the functor of Definition 1.14 and $L$ the functor of Definition 1.9. Then there exists an isometric isomorphism $L \cong L$.

Proof. The proof of this theorem requires a lemma.

Let $T^{n}$ be a toroidal group and $\delta$ the class of Borel subsets of $T^{n}$. If $\mu$ is a vector-valued measure from $\delta$ to the Banach space $X$, the semivariation of $\mu$ over a set $E \in \mathcal{S}$ is defined by

$$
\|\mu\|(E)=\sup \left\|\sum_{i=1}^{n} \alpha_{i} \mu E_{i}\right\|
$$

where the sup in (1.8) is taken over all finite collections of disjoint Borel subsets of $E$ and scalars $\alpha_{i}$.

The variation of $\mu$ over a set $E \in \mathcal{S}$ is defined by

$$
\|\| \mu\left\|(E)=\sup \sum^{N}\right\| \mu\left(E_{i}\right) \|
$$

where the supremum in (1.9) is taken for all families of disjoint sets $\left\{E_{i}\right\}_{i=1}^{N}$ such that $\cup^{N} E_{i} \subseteq E$.

Remark. If $\|\mu\| \|(E)<\infty$, then it is easy to show that $\|\mu\|(E)=\|\mu\|(E)$.

Lemma 1.0 (Dunford-Schwartz). Let $C$ be the Banach space of continuous functions on a toroidal group $T^{n}$; let $X$ be a Banach space. Then

(1) suppose that $\lambda: C \rightarrow X$ is a continuous linear operator. Then there exists a unique vector-valued measure $\mu_{\lambda}$ defined on $\delta$, with values in $X^{* *}$, such that

(i) $\left\langle x^{*}, \mu_{\lambda}(\cdot)\right\rangle$ is a regular countably additive measure for every $x^{*} \in X^{*}$;

(ii) the mapping $x^{*} \rightarrow\left\langle x^{*}, \mu_{\lambda}(\cdot)\right\rangle$ is continuous with the $X$ and $M$ topologies;

(iii) $\left\langle x^{*}, \lambda(f)\right\rangle=\left\langle x^{*}, \int f(s) d \mu_{\lambda}\right\rangle$ for every $f \in C$ and $x^{*} \in X^{*}$;

(iv) $\|\lambda\|_{\{, X]}=\left\|\mu_{\lambda}\right\|\left(T^{n}\right)$. 
The converse also holds.

(2) Suppose that $\lambda: C \rightarrow X$ is a compact linear operator. Then there exists a unique vector-valued measure $\mu_{\lambda}$ on $\delta$ having values in $X$, such that

(i) $\lambda(f)=\int f d \mu_{\lambda}$;

(ii) $\|\lambda\|_{(c, x)}=\left\|\mu_{\lambda}\right\|\left(T^{n}\right)$.

Lemma 1.0 is a statement of the general representation theorems proved in Dunford-Schwartz [10, VI, 7.2-7.3].

For every $X \in \mathcal{B}$ and $f \in L(X)$ one may now define $\tau: L(X) \rightarrow L^{1}(X)$ by

$$
\tau(f)(\varphi)=\int \varphi(t) f(t) d t \text { for every } \varphi \in C .
$$

Since $f$ is Bochner integrable the map $\tau$ is well defined. Furthermore since $\left\|T_{s} f-f\right\|_{L(X)} \rightarrow 0$ as $s \rightarrow 0$ (see Dunford-Schwartz [10, III, 12.8]) it follows that $\tau: L(X) \rightarrow L^{1}(X)$.

I shall now show that for every $\lambda$ there exists an integrable function $f_{\lambda}$ such that $\tau\left(f_{\lambda}\right)=\lambda$.

Let $\left\langle\varphi_{k}\right\rangle$ be a continuous complex-valued approximate identity such that supp $\varphi_{k}$ contracts to 0 . For every $s \in T^{n}$ define the function

$$
c_{\lambda}^{k}(s)=\lambda\left(\underline{T}_{s} \varphi_{k}\right)
$$

Since $\varphi_{k}$ is continuous for every $k,(1.10)$ is a well-defined function continuous from $T^{n}$ to $X$. Since $c_{\lambda}^{k}$ is continuous for every $k, c_{\lambda}^{k}$ is Bochner integrable for every $k$. Therefore one may write

$$
\tau\left(c_{\lambda}^{k}\right)(\varphi)=\int \varphi(s) c_{\lambda}^{k}(s) d s=\int \varphi(s) \lambda\left(\underline{I}_{s} \varphi_{k}\right) d s=\lambda\left(\varphi_{k} * \varphi\right)
$$

and furthermore

$$
\tau\left(c_{\lambda}^{k}\right)(\varphi)=\int \varphi_{k}(s) \lambda\left(I_{s} \varphi\right) d s=\int \varphi_{k}(s) T_{s} \lambda(\varphi) d s
$$

By (1.12),

$$
\begin{aligned}
\left\|\tau\left(c_{\lambda}^{k}\right)-\lambda\right\|_{\{c, X]} & =\sup _{\|\varphi\|_{c} \leq 1} \int \varphi_{k}(s)\left[T_{s} \lambda(\varphi)-\lambda(\varphi)\right] d s \\
& \leq \sup _{|s| \leq 1 / k}\left\|T_{s} \lambda-\lambda\right\|_{\{c, X]} \rightarrow 0 \text { as } k \rightarrow \infty
\end{aligned}
$$

since the supports of the $\varphi_{k}$ contract to 0 .

However, by (1.11), $\tau\left(c_{\lambda}^{k}\right)(\varphi)=\lambda\left(\varphi_{k} * \varphi\right)$ and therefore $\tau\left(c_{\lambda}^{k}\right)$ is compact as the product of a compact operator with $\lambda$. Hence $\lambda$ itself is compact as the strong limit of compact operators.

By Lemma 1.0 there exists a measure $\mu_{\lambda}$ and a sequence of measures $\mu_{\lambda}^{k}$ such that

$$
\begin{aligned}
\lambda(\varphi) & =\int \varphi d \mu_{\lambda}, & \|\lambda\|_{(c, X]} & =\left\|\mu_{\lambda}\right\|\left(T^{n}\right), \\
\tau\left(c_{\lambda}^{k}\right)(\varphi) & =\int \varphi d \mu_{\lambda}^{k}, & \left\|\tau\left(c_{\lambda}^{k}\right)\right\|_{[C, X]} & =\left\|\mu_{\lambda}^{k}\right\|\left(T^{n}\right),
\end{aligned}
$$


where $\left\langle\mu_{\lambda}^{k}\right\rangle$ is the sequence of measures defined by $d \mu_{\lambda}^{k}=c_{\lambda}^{k} d t$. Since $c_{\lambda}^{k}$ is itself a continuous function for every $k$,

$$
\left\|\tau\left(c_{\lambda}^{k}\right)\right\|_{[C, X]}=\left\|\mu_{\lambda}^{k}\right\|\left(T^{n}\right)=\int\left\|c_{\lambda}^{k}\right\| d t=\left\|c_{\lambda}^{k}\right\|_{L(X)} .
$$

Therefore the norms of $c_{\lambda}^{k}$ in $L(X)$ and $\tau\left(c_{\lambda}^{k}\right)$ in $[C, X]$ coincide. Since by (1.13) the $\tau\left(c_{\lambda}^{k}\right)$ form a Cauchy sequence in $[C, X]$, the $c_{\lambda}^{k}$ therefore form a Cauchy sequence in $L(X)$ converging to an element $f_{\lambda}$ of $L(X) ; f_{\lambda}$ is clearly a Bochner integrable function. By (1.14),

$$
\lambda(\varphi)=\int \varphi f_{\lambda} d t=\tau\left(f_{\lambda}\right)(\varphi), \quad\|\lambda\|_{C, X]}=\left\|f_{\lambda}\right\|_{L(X)} .
$$

To show that the mapping $\tau: L \rightarrow L^{1}$ defined by $\tau=\left\{\tau_{X}\right\}$ above for every $X \in \mathcal{B}$ is a natural mapping, observe that

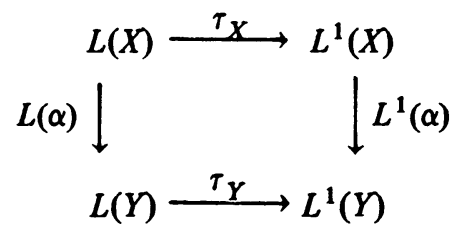

commutes, since

$$
L^{1}(\alpha) \tau_{X}(f)(\varphi)=\alpha\left(\int f \varphi d t\right)=\int(\alpha f) \varphi d t=\tau_{Y}(L(\alpha) f)(\varphi)
$$

Remark. By Proposition 32 of $\left[13\right.$, p. 145] it follows that $L^{1}(X) \subseteq M(X)$ with identity of norms.

Theorem 1.0 has a twin which applies to the functor $C$.

Definition 1.15. Let $T^{n}$ be a toroidal group and $B$ the category of Banach spaces. Define the functor $C^{\circ}: \mathcal{B} \rightarrow \mathcal{M}$ for every $X \in \mathcal{B}$ by

$$
\begin{aligned}
C^{\circ}(X) & =\left\{f: T^{n} \rightarrow X \mid f \text { continuous }\right\}, \\
\|f\|_{C^{\circ}(X)} & =\sup _{s}\|f(s)\|_{X}, \\
C^{\circ}(\alpha)(f)(t) & =\alpha f(t) \quad \forall \alpha \in[X, Y], f \in C^{\circ}(X), t \in T^{n} .
\end{aligned}
$$

Theorem 1.1. Let $T^{n}$ be a toroidal group and $B$ the category of Banach spaces. Let $C^{\circ}$ be the functor of Definition 1.15 and $C$ the functor of Definition 1.9. Then there exists an isometric isomorphism $C^{\circ} \cong C$.

Proof. I shall not prove Theorem 1.1 in detail since its proof is almost a wordfor-word repetition of that for Theorem 1.0 above. The role of Lemma 1.0 in Theorem 1.0 is here taken by a second theorem due to Dunford and Pettis.

This theorem will also be useful in other contexts. 
Theorem 1.2 (Dunford-Pettis). Let $T^{n}$ be a toroidal group and $L^{1}$ the Banach space of integrable functions on $T^{n}$. Then:

(1) Let $X=Y^{*}$ be a Banach space which is the dual of a separable Banach space $Y$. Suppose that $\lambda: L^{1} \rightarrow X$ is a continuous linear operator. Then there exists an a.e. unique weak ${ }^{*}$ measurable function $f_{\lambda}: T^{n} \rightarrow X$ such that

(i) for every $y \in Y$,

$$
\langle y, \lambda(\varphi)\rangle=\int\left\langle y, f_{\lambda}(s)\right\rangle \varphi(s) d s
$$

(ii) $\|\lambda\|_{\left[L^{\prime}, X\right]}=$ ess $\sup _{s}\left\|f_{\lambda}(s)\right\|_{X}$.

The converse is also true.

(2) Let $X$ be an arbitrary Banach space and suppose that $\lambda: L \rightarrow X$ is a compact linear operator. Then there exists an a.e. unique strongly measurable function $f_{\lambda}: T^{n} \rightarrow X$ such that

(i) $\lambda(\varphi)=\int f_{\lambda}(s) \varphi(s) d s$;

(ii) $\|\lambda\|_{\left[U^{\prime}, X\right]}=\operatorname{ess} \sup _{s}\left\|f_{\lambda}(s)\right\|_{X}$.

Theorem 1.2 may be found in [10, VI, 8.5-8.10].

Another important natural isomorphism is given by the following theorem due to Grothendieck.

Theorem 1.3 (Grothendieck). Let $T^{n}$ be a toroidal group and $\mathcal{B}$ be the category of Banach spaces, and $L^{1}$ the $L^{1}$ functor on $\mathcal{B}$. Then there exists an isometric isomorphism $\Sigma_{L^{\prime}(\mathrm{C})} \cong L^{1}$.

The proof of Theorem 1.3 may be found in Grothendieck [13, p. 60]. It involves the use of the Dunford-Pettis theorem [10, VI, 8.10] to represent the bilinear operator on $L^{1} \times X$ for $X \in \mathcal{B}$.

2. The natural mappings of harmonic analysis. As mentioned in the introduction, this paper will be concerned with the study of those natural mappings of functors defined respectively by the Fourier transform, convolution, and convolution multipliers. In this section I shall define the Fourier transform and convolution as mappings of functors in the category of Banach spaces and prove or state those theorems necessary to develop the elementary properties of these mappings.

Definition 1.16 (the Fourier transform). Let $T^{n}$ be a toroidal group and $\mathcal{B}$ the category of Banach spaces. Suppose that $D$ is the distribution functor acting on $\mathcal{B}$. For every Banach space $X \in \mathcal{B}$ and $\lambda \in D(X)$, define $\hat{\lambda}(m)=\lambda\left(e_{m}\right) \forall m$ $\in Z^{n}$ where $e_{m}$ is the function defined by $e_{m}(t)=e^{-i\langle m, t\rangle}$.

Clearly the operator ${ }^{\wedge}$ defines a linear mapping from $D(X)$ to the linear space of $X$-valued $n$-dimensional sequences on $Z^{n}$.

Definition 1.17. Let $Z^{n}$ be the group of $n$-dimensional integer lattice points and $\mathcal{B}$ the category of Banach spaces. Define 


$$
\begin{aligned}
l^{\infty}(X) & =\left\{\lambda: Z^{n} \rightarrow X \mid \lambda \text { bounded }\right\} \quad \forall X \in \mathcal{B}, \\
\|\lambda\|_{\infty}(X) & =\sup _{m \in Z^{n}}\|\lambda(m)\|_{X} \quad \forall \lambda \in l^{\infty}(X) \text { and } X \in \mathcal{B}, \\
l^{\infty}(\alpha)(\lambda) & =\alpha \circ \lambda .
\end{aligned}
$$

Clearly $l^{\infty}$ is a functor.

For the important special case in which $\lambda \in L^{\prime}(X)$ the following proposition follows immediately from Definition 1.16 and Theorem 1.0.

Proposition 1.5. Let $T^{n}$ be a toroidal group and $\mathcal{B}$ the category of Banach spaces. Let $L^{1}$ be the $L^{\prime}$ functor acting on $\mathcal{B}$. Then for every $X \in \mathcal{B}$ and $\lambda \in L^{1}(X)$,

$$
\hat{\lambda}(m)=\int f_{\lambda}(t) e^{-i\langle m, t\rangle} d t
$$

where $f_{\lambda}$ is the function corresponding to $\lambda$. Furthemore ${ }^{\wedge}$ defines a natural mapping of the functor $L^{1}$ to the functor $l^{\infty}$.

The following proposition also follows easily.

Proposition 1.6. Let $T^{n}$ be a toroidal group and $\mathcal{B}$ the category of Banach spaces. Let $D$ be the distribution functor on $\mathcal{B}$ over $T^{n}$. Then for every $X \in \mathcal{B}$ and $\lambda \in D(X), \hat{\lambda}(m)=0 \forall m \in Z^{n}$ implies $\lambda=0$.

Proof. Clearly there are very many proofs for this proposition: I shall use the very useful device of reducing the vector-valued case to the scalar case.

Let $X \in \mathcal{B}$ and $\lambda \in D(X)$, and suppose that $\hat{\lambda}(m)=0 \forall m$. Then, for every $x^{*} \in X^{*}$ and $m \in Z^{n}$,

$$
0=\left\langle x^{*}, \hat{\lambda}(m)\right\rangle=\left\langle x^{*}, \lambda\left(e_{m}\right)\right\rangle
$$

Hence by uniqueness for the case of scalar-valued distributions the distribution $\left\langle x^{*}, \lambda\right\rangle(\varphi)=\left\langle x^{*}, \lambda(\varphi)\right\rangle=0$ for every $x^{*} \in X^{*}$ and $\varphi \in C^{\infty}$. Hence $\lambda \equiv 0$.

Using the notions of vector-valued distribution and the Fourier transform of a vector-valued distribution it is now possible to extend the definition of harmonically invariant Banach space to the case of Banach spaces of vector-valued distributions.

Definition 1.18. Let $X=X_{B}$ be a Banach space consisting of distributions with values in a Banach space $B$. Let

$$
\begin{aligned}
E_{X} & =\left\{e_{m} \mid \exists \lambda \in X \ni \hat{\lambda}(m) \neq 0\right\}, \\
B E_{X} & =\left\{b e_{m} \mid b \in B, e_{m} \in E_{X}\right\}, \\
C_{X}^{\infty} & =\left\{\varphi \in C^{\infty} \mid \hat{\varphi}(m)=0 \forall m \ni e_{m} \notin E_{X}\right\} .
\end{aligned}
$$


Then I shall say that the space $X$ is harmonically invariant if

(1) $\left\|T_{s} \lambda\right\|_{X}=\|\lambda\|_{X}$

(2) $C_{X}^{\infty} \otimes B^{*}$ is a norming subspace of $X^{*}$,

(3) $B E_{X} \subseteq X$.

Clearly Definition 1.18 is constructed in strict analogy to the corresponding definition of harmonically invariant Banach space in the complex-valued case.

It is similarly possible to define vector-valued Banach modules over $M$. Clearly for every measure $\mu \in M$ and $\varphi \in C^{\infty}, \mu * \varphi \in C^{\infty}$. Let $B$ be a Banach space and $\lambda$ a distribution $\lambda \in D(B)$. Then the module product $\lambda * \mu$ is defined by duality as $\lambda * \mu(\varphi)=\lambda(\mu * \varphi)$.

As was the case for the categories $\mathcal{B}_{00}$ and $M_{00}$ one may now construct analogous categories for vector-valued distributions. Let $\mathcal{B}_{0}$ be the category of harmonically invariant Banach spaces $X$ of distributions with values in a Banach space $B_{X}$. The morphisms for $\mathcal{B}_{0}$ will consist of the continuous linear mappings $[X, Y]$. Let $M_{0}$ be the category of normal Banach modules $X$ over $M_{0}$, where $X$ again consists of vector-valued distributions, and the morphisms for $\boldsymbol{M}_{0}$ will consist of all continuous module homomorphisms $\alpha \in[X, Y]$ such that $\alpha^{*}$ : $V\left(Y^{*}\right) \rightarrow V\left(X^{*}\right)$. It is easy to see that $\mathcal{B}_{00}$ is a subcategory of $\mathcal{B}_{0}$ and $M_{00}$ is a subcategory of $M_{0}$.

For the category $\mathcal{B}_{0}$ it is also possible to define a function $W: \mathcal{B}_{0} \rightarrow \mathcal{B}_{0}$ which will be useful in what follows:

Definition 1.19. (1) Let $\mathcal{B}_{0}$ be the category of harmonically invariant Banach spaces, and define the function $W: \mathcal{B}_{0} \rightarrow \mathcal{B}_{0}$ for every $X \in \mathcal{B}_{0}$ by

$$
\begin{aligned}
W(X) & =V\left(X^{*}\right)^{*} \quad \text { where } V\left(X^{*}\right)=\mathrm{cl}_{X^{*}} C_{X}^{\infty} \otimes B^{*}, \\
\|\lambda\|_{W(X)} & =\|\lambda\|_{V\left(X^{*}\right)^{*}}
\end{aligned}
$$

(2) Let $M_{0}$ be the category of normal Banach modules over $M_{0}$, and define the functor $W: \propto M_{0} \rightarrow \mathcal{O} M_{0}$ as in (1.16) above, with also

$$
W(\alpha)=\beta^{*} \text { where } \beta=\alpha^{*} \ln \left(x^{*}\right) .
$$

The following proposition will also be important.

Proposition 1.7. Let $Y \in \mathcal{B}$. Then $\Sigma_{Y}: \mathcal{B}_{0} \rightarrow \mathcal{B}_{0}$.

Proof. Proved as Theorem 2.7 in Chapter 2.

The second elementary mapping of harmonic analysis with which I shall deal is that of tensor convolution.

Definition 1.20. Let $T^{n}$ be a toroidal group and $\mathcal{B}$ the category of Banach spaces. Let $L^{1}$ denote the $L^{1}$ functor on $\mathcal{B}$. Then for every $X, Y \in \mathcal{B}$ and $f \in L^{1}(X), g \in L^{1}(Y)$, the tensor convolution of $f$ and $g$ is defined by

$$
f * g(s)=\frac{1}{(2 \pi)^{n}} \int f(s-t) \otimes g(t) d t
$$


I shall often refer to tensor convolution simply as convolution. Clearly if $f \in L^{1}(X)$ and $g \in L^{1}(Y)$, then $f * g \in T^{n} \rightarrow X \otimes Y$.

A second definition may be given of tensor convolution for the functor $D$.

Definition 1.21. Let $T^{n}$ be a toroidal group and $\mathcal{B}$ the category of Banach spaces. Let $D$ be the distribution functor and $C^{\infty}$ the $C^{\infty}$ functor on $B$ over $T^{n}$. Then for every $X, Y \in B$ and $\lambda \in D(X)$ and $\varphi \in C^{\infty}(Y)$, the tensor convolution of $\lambda$ and $\varphi$ is defined by

$$
\begin{aligned}
\lambda * \varphi(s)= & \sum^{M} y_{i} \otimes \lambda\left(T_{s} \varphi_{i}\right) \\
& \text { for every } \varphi=\sum^{M} y_{i} \varphi_{i} \text { with } y_{i} \in Y, \varphi_{i} \in C^{\infty} \forall i
\end{aligned}
$$

and extended to all $\varphi \in C^{\infty}(Y)$ by continuity. Since every $\varphi \in C^{\infty}(X)$ is the limit in the seminorms $P_{X}^{m}$ of a uniformly absolutely convergent Fourier series $\varphi=\Sigma^{\infty} \hat{\varphi}(m) e_{m}, \lambda * \varphi$ is defined for every $\lambda \in D(X)$ and $\varphi \in C^{\infty}(Y)$. Clearly Definitions 1.20 and 1.21 coincide for $\lambda \in L^{1}(X)$.

To prove a version of Young's theorem for functors a definition will be necessary.

Definition 1.22. Let $T^{n}$ be a toroidal group and $B$ the category of Banach spaces. Suppose that $C$ is the continuity functor and $L^{1}$ is the $L^{1}$ functor on $B$ over $T^{n}$. Then for every $X, Y \in \mathcal{B}$, define bifunctors $C_{\otimes}, L_{\otimes}^{1}$, and $C_{\circledast}^{\infty}$ by

$$
\begin{aligned}
C_{\otimes}(X, Y) & =\{\lambda \in C(X \otimes Y)\}, \quad C_{\otimes}(\alpha, \beta)=\alpha \otimes \beta \circ \lambda, \\
\|\lambda\|_{C_{\otimes}(X, Y)} & =\|\lambda\|_{C(X \otimes Y)} ; \\
L_{\otimes}^{1}(X, Y) & =\left\{\lambda \in L^{1}(X \otimes Y)\right\}, \quad L_{\otimes}^{1}(\alpha, \beta)=\alpha \otimes \beta \circ \lambda, \\
\|\lambda\|_{L \downarrow(X, Y)} & =\|\lambda\|_{D(X \otimes Y)} ; \\
C_{\otimes}^{\infty}(X, Y) & =\left\{\varphi \in C^{\infty}(X \otimes Y)\right\}, \quad C_{\otimes}^{\infty}(\alpha, \beta) \varphi=\alpha \otimes \beta(\varphi), \\
P_{m}^{\otimes}(\varphi) & =\sup \left\|D^{m} \varphi\right\|_{X \otimes Y} \quad \forall m \geq 0 .
\end{aligned}
$$

(1.17), (1.18) and (1.19) are each clearly bifunctors. The following proposition will now follow easily.

Proposition 1.8 (Young's theorem for functors). Let $T^{n}$ be a toroidal group and $\mathcal{B}$ be the category of Banach spaces. Suppose that $L^{1}$ is the $L^{1}$ functor on $\mathcal{B}, C$ the continuity functor, and $D$ and $C^{\infty}$ the distribution and $C^{\infty}$ functors respectively. Then the * of tensor convolution defines a natural mapping of bifunctors

(i) $*: L^{1} \times C \rightarrow C_{\otimes}$,

(ii) $*: L^{1} \times L^{1} \rightarrow L_{\otimes}^{1}$,

(iii) $*: D \times C^{\infty} \rightarrow C_{\otimes}^{\infty}$.

Proof. Proposition 1.8 follows immediately by the same methods as in the scalar-valued case for (i), (ii). Part (iii) follows immediately from the observation 
that differentiation commutes with convolution in the tensor case also, and that $\varphi \in C^{\infty}(Y)$.

With the aid of Young's theorem it is now possible to state and prove a proposition relating convolution and the Fourier transform.

Proposition 1.9. Let $T^{n}$ be a toroidal group and $\mathcal{B}$ the category of Banach spaces. Suppose that $L^{1}$ is the $L^{1}$ functor and $D$ and $C^{\infty}$ are the distribution and $C^{\infty}$ functors on $B$ over $T^{n}$. Then:

(I) For every $X, Y \in \mathcal{B}$ and $f \in L^{\prime}(X), g \in L^{\prime}(Y)$,

$$
(f * g)^{\wedge}(m)=\hat{f}(m) \otimes \hat{g}(m)
$$

(II) For every $X, Y \in \mathcal{B}$ and $\lambda \in D(X), \varphi \in C^{\infty}(Y)$,

$$
(\lambda * \varphi)^{\wedge}(m)=\hat{\lambda}(m) \otimes \hat{\varphi}(m)
$$

Proof. (I) Since there does not exist a satisfactory version of the Fubini-Tonelli theorem for vector-valued integrals, it is necessary to approximate $f$ and $g$ by continuous Banach-space valued functions $f_{k} \rightarrow f$ in $L^{\prime}(X)$ and $g_{k} \rightarrow g \in L^{\prime}(Y)$. Since $f$ and $g$ are Bochner integrable and hence a.e. separably valued this may clearly be done. Hence it is enough to show the theorem for $f$ and $g$ continuous whence (1.20) follows in the general case. However for $f$ and $g$ continuous,

$$
\begin{aligned}
(f * g)^{n}(m) & =\frac{1}{(2 \pi)^{n}} \int f * g(s) e^{-i\langle m, s\rangle} d s \\
& =\frac{1}{(2 \pi)^{n}} \int e^{-i\langle m, s\rangle}\left\{\frac{1}{(2 \pi)^{n}} \int f(s-t) \otimes g(t) d t\right\} d s \\
& =\frac{1}{(2 \pi)^{n}} \int f(s-t) e^{-i\langle m, s-t\rangle} \otimes\left(\frac{1}{(2 \pi)^{n}} \int g(t) e^{-i\langle m, t\rangle} d t\right) d s \\
& =\frac{1}{(2 \pi)^{n}} \int f(s-t) e^{-i\langle m, s-t\rangle} \otimes \hat{g}(m) d s=\hat{f}(m) \otimes \hat{g}(m)
\end{aligned}
$$

just as in the scalar-valued case.

(II) Let $X, Y \in \mathcal{B}$ and $\lambda \in D(X), \varphi \in C^{\infty}(X)$. Then $\varphi=\Sigma_{\mu}^{\infty} \hat{\varphi}(\mu) e$ where $\hat{\varphi}(\mu) \in Y \forall \mu$ and the Fourier series $\Sigma^{\infty} \hat{\varphi}(\mu) e_{\mu}$ is uniformly and absolutely convergent together with all of its derivatives. Then by the continuity of $\lambda$ and the Definitions 1.16, 1.21 of the Fourier transform and convolution

$$
\begin{aligned}
\int \lambda * \varphi(s) e_{m}(s) d s & =\int \sum \hat{\varphi}(\mu) \otimes \lambda\left(T_{s} e_{\mu}\right) e_{m}(s) d s \\
& =\sum^{\infty} \hat{\varphi}(\mu) \otimes \lambda\left(e_{\mu}\right) \int e_{\mu}(s) e_{m}(s) d s=\hat{\varphi}(m) \otimes \hat{\lambda}(m) .
\end{aligned}
$$




\section{CHAPTER 2: THE TRANSLATION OPERATORS}

In this section I shall develop the basic properties of the four mappings $\Delta, \nabla$, $\tau, \Lambda$, which will be of very much importance in the next chapters. These mappings provide a technical apparatus to transform theorems on natural mappings of functors on the category $\mathcal{B}$ into cognate theorems on the continuity of integral operators acting on harmonically invariant spaces in the category $\mathcal{B}_{0}$; in particular they allow the process discussed in the introduction to be carried out for very general spaces of distributions.

The following proposition will be used in a basic way.

Proposition 2.0. Let $T^{n}$ be a toroidal group and $C_{n}^{\infty}, C_{2 n}^{\infty}$ be the metric spaces of complex-valued $C^{\infty}$ functions and $D_{n}, D_{2 n}$ the distribution functors on $T^{n}$ and $T^{2 n}=T^{n} \times T^{n}$ respectively. Then:

(1) $C_{n}^{\infty} \otimes C_{n}^{\infty} \cong C_{2 n}^{\infty}$.

(2) $D_{2 n} \cong D_{n} \circ D_{n} \cong D_{n} \otimes D_{n}$.

(3) For every $Y \in \mathcal{B}$, there exists a natural isomorphism $\Sigma_{Y} \circ D_{n} \cong D_{n} \circ \Sigma_{Y}$ $\cong \Sigma_{Y \otimes D_{n}}$.

(4) There exist natural isomorphisms $D_{n} \cong \operatorname{Hom}_{\otimes}\left[D_{n}, D_{n}\right] \cong \operatorname{Hom}_{\otimes}\left[C_{n}^{\infty}, D_{n}\right]$.

Proof. (1) The proof of (1) may be found in L. Schwartz [36, Proposition I, p. 17, Chapter II].

(2) This part follows very easily from part (1) by virtue of the properties of the tensor product shown for Banach spaces in Proposition 1.3 above. Let $X \in \mathcal{B}$. Then

$$
D_{2 n}(X)=\left[C_{2 n}^{\infty}, X\right] \cong\left[C_{n}^{\infty} \otimes C_{n}^{\infty}, X\right] \cong\left[C_{n}^{\infty},\left[C_{n}^{\infty}, X\right]\right]=D_{n} \circ D_{n}(X) .
$$

(3) Let $X$ be a Banach space and define, for every $y \otimes \lambda \in Y \otimes D_{n}(X)$ and $\boldsymbol{X} \in \mathcal{B}$,

$$
i_{x}(y \otimes \lambda)=\lambda \otimes y
$$

(2.0) is clearly a continuous isomorphism after extension by linearity.

Now observe that since $D_{n}$ is a nuclear space and $\otimes$ is the projective tensor product (cf. [13, Definition 2, p. 32]) it follows by [13, Theorem 6, p. 34] that $D_{n}(X)=D_{n} \otimes X$. Hence

$$
D_{n}(X) \otimes Y=D_{n} \otimes X \otimes Y=D_{n}(X \otimes Y)
$$

Let $j_{X}(x \otimes y)=y \otimes x$, and extend $j_{X}$ by linearity. Then $j_{X}$ is also continuous and isometric and $D_{n}\left(j_{X}\right): D_{n}(X \otimes Y) \rightarrow D_{n}(Y \otimes X)$. One may therefore define the mapping

$$
\gamma_{X}=i_{X} \circ j_{X}: \Sigma_{Y} D_{n}(X) \rightarrow D_{n} \Sigma_{Y}(X)
$$


Clearly also the mapping $\gamma=\left\{\gamma_{X}\right\}$ is natural, since for every $\boldsymbol{X}, \boldsymbol{Y}$, and $\alpha \in[X, Z]$, the diagram

$$
\begin{array}{cc}
\Sigma_{Y} \circ D_{n}(X)=Y \otimes D_{n}(X) \stackrel{\gamma_{X}}{\longrightarrow} D_{n} \circ \Sigma_{Y}(X) \\
1_{Y} \otimes D_{n}(\alpha) \mid & \left.\right|_{D_{n}\left(1_{Y} \otimes \alpha\right)} \\
\Sigma_{Y} \circ D_{n}(Z)=Y \otimes D_{n}(Z) \underset{\gamma_{Z}}{\longrightarrow} D_{n} \circ \Sigma_{Y}(Z)
\end{array}
$$

commutes:

$$
\begin{aligned}
D_{n}\left(1_{Y} \otimes \alpha\right) \gamma_{X}(y \otimes \lambda) & =1_{D_{n}} \otimes 1_{Y} \otimes \alpha(\lambda \otimes y \otimes x) \\
& =\gamma_{Z}\left(1_{Y} \otimes 1_{D_{n}} \otimes \alpha\right)(y \otimes \lambda \otimes x)=\gamma_{Z}\left(y \otimes D_{n}(\alpha) \lambda\right) \\
& =\gamma_{Z} \circ 1_{Y} \otimes D_{n}(\alpha)(y \otimes \lambda) .
\end{aligned}
$$

(4) I shall show the existence of an isomorphism $\operatorname{Hom}_{\odot}\left[D_{n}, D_{n}\right] \cong D_{n}$. The second part follows easily in the same way.

Observe that by Definition 1.13 and part (3) above, there exist natural isomorphisms $\operatorname{Hom}_{\circledast}\left[D_{n}, D_{n}\right](Y)=\left[D_{n}, D_{n} \circ \Sigma_{Y}\right]$.

Let $H \in\left[D_{n}, D_{n}(Y)\right]$. Since $H$ commutes with elements of $M, e_{m} * H\left(e_{m}\right)$ $=H\left(e_{m}\right)$. Let $\varphi(m)=H\left(e_{m}\right)^{\wedge}(m)$. Then

$$
H\left(e_{m}\right)=H\left(e_{m}\right)^{\wedge}(m) e_{m}=\varphi(m) e_{m} \quad \forall m
$$

where $\varphi(m) \in Y$ for every $m$.

Furthermore for every trigonometric polynomial $p$ and $\lambda \in D_{n}, p * \lambda$ is a trigonometric polynomial. Therefore

$$
\begin{aligned}
& H(p * \lambda)^{\wedge}(m)=\hat{p}(m) H(\lambda)^{\wedge}(m), \\
& H(p * \lambda)^{\wedge}(m)=\varphi(m)(p * \lambda)^{\wedge}(m)=\varphi(m) \hat{p}(m) \hat{\lambda}(m) .
\end{aligned}
$$

Since (2.2) holds for every trigonometric polynomial, it follows that $H(\lambda)^{\wedge}(m)$ $=\varphi(m) \hat{\lambda}(m) \forall m$.

I shall now show that $\varphi$ is the Fourier transform of a distribution $h \in D_{n}(Y)$. From this fact it will be possible to define the isomorphism $i_{Y}: \operatorname{Hom}_{\odot}\left[D_{n}, D_{n} \Sigma_{Y}\right]$ $\rightarrow D_{n}(Y)$.

To do so I shall first prove a lemma.

Lemma 2.0.1. Let $Y$ be a Banach space and $\lambda \in D_{n}(Y)$. Then:

(1) There exists an integer $m$ and $a$ constant $k$ such that, for every $\varphi \in C_{n}^{\infty}$, 


$$
\|\lambda(\varphi)\|_{Y} \leq k \sup _{0 \leq p \leq m}\left\|D^{p} \varphi\right\|_{\infty} .
$$

(2) There exists an integer $k$ such that $\|\hat{\lambda}(m)\|_{Y} \leq|m|^{k}$.

Proof. This proof is standard. Suppose that no inequality of the form (2.3) held. Then there would exist a sequence $\left\langle\varphi_{k}\right\rangle \in C_{n}^{\infty}$ such that $\varphi_{k} \neq 0 \forall k$ and

$$
\left\|\lambda\left(\varphi_{k}\right)\right\|_{Y}>k \cdot \sup _{0 \leq p \leq k}\left\|D^{p} \varphi_{k}\right\|_{\infty} .
$$

Since $\varphi_{k} \neq 0$ by (2.4), $\alpha_{k}=\sup _{0 \leq p \leq k}\left\|D^{p} \varphi_{k}\right\|_{\infty}>0$. Let $\psi_{k}=\left(k \alpha_{k}\right)^{-1} \varphi_{k}$. Then $\varphi_{k} \in C_{n}^{\infty}$ and $\sup _{0 \leq p \leq k}\left\|D^{p} \psi_{k}\right\|_{\infty}=k^{-1}$; hence $\psi_{k} \rightarrow 0$ in $C^{\infty}$. However

$$
\left\|\lambda\left(\psi_{k}\right)\right\|_{Y}=\left(k \alpha_{k}\right)^{-1}\left\|\lambda\left(\varphi_{k}\right)\right\|_{Y}>1
$$

Since $\lambda: C^{\infty} \rightarrow Y$ is continuous, (2.4) is impossible and the inequality (2.3) is true.

(2) follows immediately from (1) on setting $\varphi=e_{m}$ for each exponential $e_{m}$.

By Lemma 2.0.1, $\varphi$ is the Fourier transform of $h \in D_{n}(Y)$ if and only if $\langle\varphi(m)\rangle$ is a tempered sequence in $Y$. Suppose that $\langle\varphi(m)\rangle$ is not tempered. Then there exists a sequence $\left\langle m_{k}\right\rangle$ such that $\left|m_{k}\right| \rightarrow \infty$ and $\left\|\varphi\left(m_{k}\right)\right\|_{r}>\left|m_{k}\right|^{k}$. Let $\psi(x)$ $=\sum\left|m_{k}\right|^{-k / 2} e^{j\left\langle m_{k}, x\right\rangle} . \psi$ is clearly in $C^{\infty}$; however

$$
\left\|\varphi\left(m_{k}\right) \hat{\psi}\left(m_{k}\right)\right\|_{Y}>\left|m_{k}\right|^{k / 2}
$$

which contradicts the fact that $H(\lambda)^{\wedge}=\varphi \cdot \hat{\lambda}$ is a distribution in $D_{n}(Y)$ and therefore tempered.

Let ${ }^{\bar{a}}$ denote the inverse Fourier-Schwartz transform of an $m$-dimensional sequence in $Z^{n}$, and define $i_{Y}(H)=\hat{\varphi}=h$ where $h$ is the distribution in $D_{n}(Y)$ generated by $\varphi$.

Clearly $i=\left\{i_{Y}\right\}$ is a monomorphism; it is also clear that $i$ is onto. To see that $i$ is natural, observe that the diagram

$$
\begin{gathered}
\operatorname{Hom}_{\otimes}\left[D_{n}, D_{n}\right](Y) \stackrel{. i_{Y}}{\longrightarrow} D_{n}(Y) \\
\operatorname{Hom}_{\otimes}\left[D_{n}, D_{n}\right](\alpha) \\
\operatorname{Hom}_{\Theta}\left[D_{n}, D_{n}\right](Z) \stackrel{ }{i_{Z}} D_{n}(Z)
\end{gathered}
$$

clearly commutes since the Fourier transform ${ }^{\wedge}$ and its inverse ${ }^{\overline{ }}$ on $Z^{n}$ are both natural mappings.

Definition 2.0. Let $C_{n}^{\infty}, C_{2 n}^{\infty}$ be the metric spaces of complex-valued $C^{\infty}$ functions on $T^{n}$ and $T^{2 n}$. Define 


$$
\begin{aligned}
\Delta \varphi(x, y) & =\frac{1}{(2 \pi)^{n}} \int \varphi(x+s, y-s) d s \quad \forall \varphi \in C_{2 n}^{\infty}, \\
\Lambda \varphi(x) & =\frac{1}{(2 \pi)^{n}} \int \varphi(s, x-s) d s, \\
\nabla \varphi & =(I-\Delta) \varphi ; \\
\tau \varphi(x, y) & =\varphi(x+y) \quad \forall \varphi \in C_{n}^{\infty} .
\end{aligned}
$$

It is now possible to define the mappings $\Delta, \nabla, \Lambda$, and $\tau$ on spaces of distributions.

Definition 2.1. Let $D_{n}, D_{2 n}$ be the distribution functors over $\mathcal{B}$ on the toroidal groups $T^{n}, T^{2 n}$ respectively. Define natural mappings $\Delta, \nabla, \Lambda, \tau, \Delta^{*}$ for every $X \in \mathcal{B}$ by

$$
\begin{aligned}
\Delta_{X} \lambda(\varphi) & =\lambda(\Delta \varphi), & & \forall \lambda \in D_{2 n}(X) \text { and } \varphi \in C_{2 n}^{\infty}, \\
\nabla_{X} \lambda & =\left(I-\Delta_{X}\right) \lambda, & & \\
\Lambda_{X} \lambda(\varphi) & =\lambda(\tau \varphi), & & \forall \lambda \in D_{2 n}(X), \varphi \in C_{n}^{\infty}, \\
\tau_{X} \lambda(\varphi) & =\lambda(\Lambda \varphi), & & \forall \lambda \in D_{n}(X), \varphi \in C_{2 n}^{\infty}, \\
\Delta_{X}^{*} H & =\Delta_{X \otimes Z}\left(H_{Z} \otimes 1_{D_{n}}\right) \Delta_{Z}, & & \forall H \in \operatorname{Hom}_{\otimes}\left[D_{n}, D_{n}\right](X), \quad \forall Z \in \mathcal{B}, \\
\Lambda_{X}^{*} H & =\Lambda_{X \otimes Z}\left(H_{Z} \otimes 1_{D_{n}}\right) \tau_{Z} . & &
\end{aligned}
$$

Remark. It is clear from Definition 2.1 that, for $X \in \mathcal{B}$,

$$
\begin{aligned}
& \Delta_{X}: D_{2 n}(X) \rightarrow D_{2 n}(X), \\
& \nabla_{X}: D_{2 n}(X) \rightarrow D_{2 n}(X), \\
& \Lambda_{X}: D_{2 n}(X) \rightarrow D_{n}(X), \\
& \tau_{X}: D_{n}(X) \rightarrow D_{2 n}(X), \\
& \Delta_{X}^{*}: \operatorname{Hom}_{\otimes}\left[D_{n}, D_{n}\right](X) \rightarrow \operatorname{Hom}_{\otimes}\left[D_{n} D_{n}, D_{n} D_{n}\right](X), \\
& \Lambda_{X}^{*}: \operatorname{Hom}_{\otimes}\left[D_{n}, D_{n}\right](X) \rightarrow \operatorname{Hom}_{\otimes}\left[\Lambda D_{n} D_{n}, \Lambda D_{n} D_{n}\right](X) .
\end{aligned}
$$

The last two relationships follow from Proposition 2.0.

The following theorem states the fundamental properties of the six mappings above acting on the distribution functors $D_{n}$ and $D_{2 n}$.

Theorem 2.0. Let $\mathcal{B}$ be the category of Banach spaces and $D_{n}, D_{2 n}$ the distribution functors on $B$ over $T^{n}, T^{2 n}$ respectively. Then:

(1) Let $J$ be the natural embedding of $\nabla D_{n} D_{n}$ in $D_{n} D_{n}$. Then

$$
0 \rightarrow \nabla D_{n} D_{n} \stackrel{\jmath}{\rightarrow} D_{n} D_{n} \stackrel{\Delta}{\rightarrow} D_{n} D_{n} \rightarrow 0
$$


is split exact.

(2) In the diagram

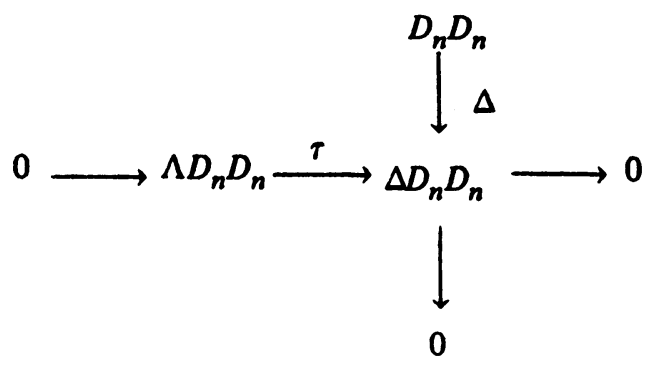

all sequences are exact.

$$
\begin{aligned}
& 0 \rightarrow \operatorname{Hom}_{\otimes}\left[D_{n}, D_{n}\right] \stackrel{\Delta^{\bullet}}{\rightarrow} \operatorname{Hom}_{\otimes}\left[D_{n} D_{n}, D_{n} D_{n}\right], \\
& 0 \rightarrow \operatorname{Hom}_{\circledast}\left[D_{n}, D_{n}\right] \stackrel{\Lambda^{\bullet}}{\rightarrow} \operatorname{Hom}_{\otimes}\left[\Lambda D_{n} D_{n}, \Lambda D_{n} D_{n}\right]
\end{aligned}
$$

are exact.

(4) All mappings of functors in (1), (2), (3) are natural.

Remark. Let $H$ be a complex-valued distribution. The import of Theorem 2.0, in particular parts (3) and (4), is that the mapping $\tau$ commutes with any convolution operators $H \in\left[D_{n}, D_{n}\right]$, and hence with any operator $H \in[F, F]$ for a large class of functors. Thus $\Lambda H * \tau \lambda=H * \lambda$ and therefore $H * \tau \lambda=$ $\tau(H * \lambda)$. By part (3) of Theorem 2.0 one is enabled to transform multiplier theorems for mappings of functors $[F, G]$ into theorems on mappings $[\Lambda F, \Lambda G]$. Essentially parts (3) and (4) say that any mappings in $\operatorname{Hom}_{\circledast}[F, G]$ correspond exactly to mappings in $\operatorname{Hom}_{\circledast}[\Lambda F, \Lambda G]$, and therefore will be mappings for any spaces of the form $\Lambda F(X), \Lambda G(X)$ for any $X \in \mathcal{B}_{0}$.

Proof. (1) It is clear that $\Delta$ is onto $\Delta D_{n} D_{n}$ and that the embedding map $J_{X}$ is a monomorphism. Therefore it is only necessary to show that $\nabla D_{n} D_{n}(X)$ is exactly the kernel of $\Delta_{x}$.

Observe that by Definition 2.1 of $\Delta_{X}$ and the fact that $D_{2 n}(X) \cong D_{n} D_{n}(X)$, for every $\lambda \in D_{2 n}(X) \cong D_{n} D_{n}(X)$,

$$
\Delta_{X}^{2} \lambda(\varphi)=\lambda\left(\Delta^{2} \varphi\right)
$$

and by Definition 2.0

$$
\begin{aligned}
\Delta^{2} \varphi(x, y) & =\frac{1}{(2 \pi)^{2 n}} \iint \varphi(x+s+t, y-s-t) d s d t \\
& =\frac{1}{(2 \pi)^{n}} \int \varphi(x+u, y-u) d u=\Delta \varphi(x, y)
\end{aligned}
$$


Hence $\nabla \varphi=(I-\Delta) \varphi \in \operatorname{Ker} \Delta$.

Let $\Delta_{X} \lambda(\varphi)=0$. Then $\lambda(\Delta \varphi)=0$. Clearly $\Delta$ is continuous from $C_{2 n}^{\infty}$ to $C_{2 n}^{\infty}$, and therefore by (2.7) above, $\Delta$ is a projection. Hence

$$
\lambda(\varphi)=\lambda((I-\Delta) \varphi)+\lambda(\Delta \varphi)=\lambda((I-\Delta) \varphi)=\nabla_{X} \lambda(\varphi) .
$$

Thus $\lambda=\nabla_{X} \lambda \in \nabla D_{n} D_{n}(X)$. Hence $\operatorname{Ker} \Delta_{X}=\nabla_{X} D_{n} D_{n}(X)$. To see that the sequence (1) is split exact, it is now only necessary to observe that $\lambda=\nabla_{X} \lambda$ $\oplus \Delta_{X} \lambda$ forms a direct sum decomposition of $D_{n} D_{n}(X)=\nabla_{X} D_{n} D_{n}(X) \oplus \Delta_{X} D_{n} D_{n}$.

(2) Let $X \in \mathcal{B}$. It is clear again that the vertical sequence $D_{n} D_{n}(X)$ $\rightarrow \Delta_{x} \Delta_{X} D_{n} D_{n}(X) \rightarrow 0$ is exact since $\Delta_{X} D_{n} D_{n}(X)$ is just the image of $D_{n} D_{n}(X)$ under $\Delta_{X}$. To show that the horizontal sequence is exact, it is only necessary to show that $\tau_{X}$ is onto and one to one.

To show that $\tau_{X}$ is onto, let $\lambda \in \Delta_{X} D_{n} D_{n}(X)$. Then $\lambda=\Delta_{X} \lambda$. Let $\lambda_{0}=\Lambda_{X} \lambda$. For every $\varphi \in C_{n}^{\infty}$,

$$
\tau_{X} \lambda_{0}(\varphi)=\tau_{X} \Lambda_{X} \lambda(\varphi)=\Delta_{X} \lambda(\Lambda \varphi)=\lambda(\tau \Lambda \varphi)
$$

However

$$
\begin{aligned}
\tau \Lambda \varphi(x, y) & =\frac{1}{(2 \pi)^{n}} \tau \int \varphi(s, x-s) d s=\frac{1}{(2 \pi)^{n}} \int \varphi(s, x+y-s) d s \\
& =\frac{1}{(2 \pi)^{n}} \int \varphi(x+s, y-s) d s=\Delta \varphi .
\end{aligned}
$$

Hence $\tau_{X} \lambda_{0}(\varphi)=\lambda(\Delta \varphi)=\Delta_{X} \lambda(\varphi)=\lambda(\varphi)$ and $\tau_{X}$ is onto.

To show that $\tau_{X}$ is a monomorphism, observe first that

$$
\Lambda \tau \varphi(x)=\frac{1}{(2 \pi)^{n}} \int \varphi(s+x-s) d s=\varphi(x)
$$

and therefore $\Lambda$ is a left inverse for $\tau$. Suppose now that $\tau_{X}(\lambda)=0$. Then $\langle\Lambda \varphi, \lambda\rangle=0$ for every $\varphi \in C_{2 n}^{\infty}$, since $\tau: C_{n}^{\infty} \rightarrow C_{2 n}^{\infty},\langle\varphi, \lambda\rangle=\langle\Lambda \tau \varphi, \lambda\rangle=0$ for every $\varphi \in C_{n}^{\infty}$ and hence $\lambda=0$.

(3) To show that $\Delta_{X}^{*}$ is a monomorphism, observe first that by Proposition 2.0, $D_{n} \circ \Sigma_{Y} \cong \Sigma_{D_{n} \otimes Y}$. Hence there exists a natural algebraic isomorphism $\operatorname{Hom}_{\odot}\left[D_{n}, D_{n}\right](Y) \cong\left[D_{n}, D_{n} \Sigma_{Y}\right] \cong\left[\Sigma_{D_{n}}, \Sigma_{D_{n} \otimes Y}\right]$. Furthermore it is easy to show that the mapping $i_{Z}\left(H_{Z}\right)=H_{\mathrm{C}}$ with inverse $i_{z}\left(H_{\mathrm{C}}\right)=H_{\mathrm{C}} \otimes 1_{\mathrm{Z}}$ defines a natural algebraic isomorphism

$$
\left[\Sigma_{D_{n}}, \Sigma_{D_{n} \otimes Y}\right] \stackrel{i_{Z}}{=}\left[D_{n}, D_{n} \otimes Y\right]=\left[D_{n}, D_{n}(Y)\right]
$$

where in the last two terms of $(2.8), D_{n}=D_{n}(C)$ and $D_{n}(Y)$ are the respective spaces of distributions.

It will therefore be sufficient to show (4) for the case in which the Banach space $Z$ is simply the space $\mathbf{C}$ of complex numbers. 
Suppose that $Y \in \mathcal{B}$ and $H \in\left[D_{n}, D_{n}(Y)\right]$ and that $\Delta_{Y}^{*} H=\Delta_{Y}\left(H \otimes 1_{D_{n}}\right) \Delta_{C}$. Then in particular for every distribution $\lambda=e_{m}^{x} e_{m}^{y} \in D_{2 n}=D_{n}^{x} \otimes D_{n}^{y}$,

$$
\Delta_{Y} H \otimes 1_{D_{n}} \Delta_{\mathrm{C}}\left(e_{m}^{x} e_{m}^{y}\right)=0 .
$$

Since $\Delta_{c}\left(e_{m}^{x} e_{m}^{y}\right)(x, y)=(2 \pi)^{-n} \int e_{m}(x+s) e_{m}(y-s) d s=e_{m}(x) e_{m}(y)$,

$$
0=\Delta_{Y}\left(H \otimes 1_{D_{n}}\right)\left(e_{m}^{x} e_{m}^{y}\right)=\Delta_{Y}\left(H\left(e_{m}^{x}\right) e_{m}^{y}\right)=\Delta_{Y} A(m) e_{m}^{x} e_{m}^{y}=\mathscr{H}(m) e_{m}^{x} e_{m}^{y} .
$$

Hence $\hat{H}(m)=0 \forall m \in Z^{n}$. Since $\hat{H}$ is the Fourier-Schwartz transform of the distribution in $D_{n}(Y)$ corresponding to $H$ by the monomorphism $\operatorname{Hom}_{\circledast}\left[D_{n}, D_{n}\right]$ $\rightarrow D_{n}$ it follows that $H \equiv 0$ and hence $\Delta^{*}$ is a monomorphism.

Finally it follows immediately from (2) that the second sequence in (3) is a monomorphism.

(4) Since the proofs that the mappings of Theorem 2.0 are natural are closely analogous in each case, I shall only prove that $\Delta$ is natural. To do so it is only necessary to observe that the diagram

$$
\begin{gathered}
D_{2 n}(X) \stackrel{\Delta_{X}}{\longrightarrow} D_{2 n}(X) \\
D_{2 n}(\alpha) \downarrow \\
D_{2 n}(Y) \underset{\Delta_{Y}}{\longrightarrow} \cdot D_{2 n}(Y)
\end{gathered}
$$

commutes, since for every $\lambda \in D_{2 n}(X)$ and $\varphi \in C_{2 n}^{\infty}$,

$$
\Delta_{Y} D_{2 n}(\alpha) \lambda(\varphi)=\Delta_{Y}(\alpha \circ \lambda)(\varphi)=\alpha(\lambda(\Delta \varphi))=\alpha\left(\Delta_{X} \lambda(\varphi)\right)=D_{2 n}(\alpha) \Delta_{X} \lambda(\varphi) .
$$

Let $K$ be a functor on the category $B$ over the toroidal group $T^{n}$. I shall say that $K$ is between the functor $C_{n}^{\infty}$ and $D_{n}$ if the embedding monomorphisms $C^{\infty} \subseteq \eta \quad K \subseteq \eta^{\prime} D_{n}$ are natural.

The following proposition will be important to interpreting the results of Theorem 2.0 for the rest of this section.

Proposition 2.1. Let $K$ be a functor over $T^{n}$ on the category $\mathcal{B}$, and suppose that $K$ is between $C_{n}^{\infty}$ and $D_{n}$. Then:

(1) For every Banach space $X \in B$ consisting of distributions on $T^{n}$ with values in a Banach space $B \in \mathcal{B}$, and every $Y \in \mathcal{B}$, there exists a monomorphism $\mu_{Y}$,

$$
K(X \otimes Y) \stackrel{\mu_{Y}}{\subseteq} D_{2 n}(B \otimes Y) .
$$

(2) Let $K$ be a functor $K: B \rightarrow \triangle M_{0}$. Then there exists a natural monomorphism $\mu^{*}$,

$$
\operatorname{Hom}_{\otimes}[K, K] \stackrel{\mu^{*}}{\subseteq} \operatorname{Hom}_{\circledast}\left[D_{n}, D_{n}\right] .
$$


Proof. Part (1) is an immediate consequence of Proposition 2.0 together with the facts that $X$ consists of distributions on $T^{n}$, and $K$ is a subfunctor of $D_{n}$ :

$$
\begin{aligned}
K(X \otimes Y) & \subseteq D_{n}(X \otimes Y) \cong D_{n} \otimes X \otimes Y \subseteq D_{n} \otimes D_{n} \otimes B \otimes Y \\
& \cong D_{2 n} \otimes B \otimes Y .
\end{aligned}
$$

(2) Since the functor $K$ is between $C^{\infty}$ and $D_{n}$, the embedding monomorphisms $\eta, \eta^{\prime}$ are both continuous. For every $Y \in \mathcal{B}$ and $H \in \operatorname{Hom}_{\otimes}[K, K](Y)$ define the mapping $\mu_{1}^{*}: \operatorname{Hom}_{\otimes}[K, K] \rightarrow \operatorname{Hom}_{\otimes}\left[C^{\infty}, D_{n}\right]$ by

$$
\mu_{Y}^{*}\left(H_{X}\right)=\eta_{X \otimes Y}^{\prime} \circ H_{X} \circ \eta_{X} .
$$

To show that $\mu^{*}$ is a monomorphism, suppose that $\mu_{Y}^{*}\left(H_{X}\right) \equiv 0$ for $X, Y \in \mathcal{B}$. Then since $\eta_{X \otimes Y}^{\prime}$ is a monomorphism $H_{X} \circ \eta_{X} \equiv 0$, and therefore $H_{X}\left(f_{0}\right)=0$ for every $f_{0} \in C^{\infty}(X)$. Since $K: \mathcal{B} \rightarrow \propto M_{0}, H_{X}$ is a module homomorphism over $L^{1}$. Hence for every $\varphi \in C^{\infty}$ and $f \in K(X), \varphi * H_{X}(f)=H_{X}(\varphi * f)=0$. Therefore $H_{X}(f)=0$ for every $f \in K(X)$ and $H_{X}=0$.

Since the mappings $\eta^{\prime}$ and $\eta$ in (2.10) are both natural it follows that $\mu^{*}$ is natural. Since by Proposition 2.0 there exists an isomorphism $i: \operatorname{Hom}_{\otimes}\left[C^{\infty}, D_{n}\right]$ $\cong \operatorname{Hom}_{\otimes}\left[D_{n}, D_{n}\right]$, the mapping $\mu^{*}=i \circ \mu_{1}^{*}$ defines a natural monomorphism $\mu^{*}: \operatorname{Hom}_{\otimes}[K, K] \subseteq \operatorname{Hom}_{\circledast}\left[D_{n}, D_{n}\right]$.

Let $K$ be a functor between $C^{\infty}$ and $D_{n}$ acting on the category $\mathcal{B}$, and suppose that $X \in \mathcal{B}$ is a Banach space consisting of distributions on $T^{n}$ with values in a Banach space $B \in \mathcal{B}$. Since, by Proposition 2.1, $K(X) \subseteq D_{2 n}(B)$, the mappings $\Delta, \nabla, \Lambda, \tau$ define mappings on $K(X)$ by restriction. One may therefore make a definition:

Definition 2.2. Let $K$ be a functor between $C^{\infty}$ and $D_{n}$ acting on the category $\mathcal{B}$. For every Banach space $X \in \mathcal{B}$ consisting of distributions on $T^{n}$ with values in a Banach space $B \in \mathcal{B}$, define restrictions of $\Delta, \nabla, \Lambda, \tau$ to $K(X)$ by

$$
\begin{array}{ll}
\Delta_{X}=\left.\Delta_{B}\right|_{K(X)}, & \nabla_{X}=\left.\nabla_{B}\right|_{K(X)}, \\
\Lambda_{X}=\left.\Lambda_{B}\right|_{K(X)}, & \tau_{X}=\left.\tau_{B}\right|_{\Lambda_{X} K(X)} .
\end{array}
$$

If $X$ is a Banach space of distributions with values in $B$, I shall regard the Banach space $B$ as uniquely determined by $X$. It will then follow that given a functor $K$ and a Banach space $X$ of $B$-valued distributions, the meaning of $\Delta_{X}$, $\nabla_{X}, \Lambda_{X}, \tau_{X}$ is unambiguously assigned.

For the rest of this paper, when the functor $K$ and the Banach space $X$ may be clearly understood from context, I shall often write $\Delta, \nabla, \Lambda, \tau$ for the mappings $\Delta_{X}, \nabla_{x}, \Lambda_{X}, \tau_{x}$

In the forms given by Definition 2.2 and Definition 2.5 (to come) the mappings of Definition 2.1 and Theorem 2.0 will be used extensively throughout this paper. Their properties however will depend strongly on the categories on which they act. The weakest properties which are still of interest are given in the following definition and theorem for the category $\mathcal{B}_{\omega}$. 
Definition 2.3. Let $T^{n}$ be a toroidal group and $L^{1}$ the $L^{1}$ functor on the category $\mathcal{B}_{\omega}$. Suppose that $K$ is a norm subfunctor of $L^{l}$ and $\delta^{\circ}$, the operator of translation by $t$ acting on $X \in \mathcal{B}_{\omega}$. Define, for every $X \in \mathcal{B}_{\omega}$,

$$
\begin{aligned}
& \Delta K(X)=\left\{f \in K(X) \mid \delta^{\circ}, f_{-t} \text { is Bochner integrable in } L^{1}(X)\right\} \\
& \|f\|_{\Delta K(X)}=\frac{1}{(2 \pi)^{n}} \int\left\|\delta^{\circ}, f_{-\imath}\right\|_{K(X)} d t .
\end{aligned}
$$

Clearly $\Delta K$ defines a functor on the category $\mathcal{B}_{\omega}$ (in $\mathcal{B}_{\omega}$ the only mappings are the identity).

It is important to note that $\delta^{\circ}$, may not be continuous in $t$ when acting on general spaces $X \in \mathcal{B}$; again, $\Delta K(X)$, although clearly a subspace of $K(X)$, may not be a closed subspace.

The properties of the mappings $\Delta$ and $\tau$ on $\Delta K$ are given in the following theorem.

Theorem 2.1. Let $K$ be a norm subfunctor of the $L^{1}$ functor on $\mathcal{B}_{\omega}$ over $T^{n}$. Then for every $X \in \mathcal{B}_{\omega}$, all sequences in the diagram

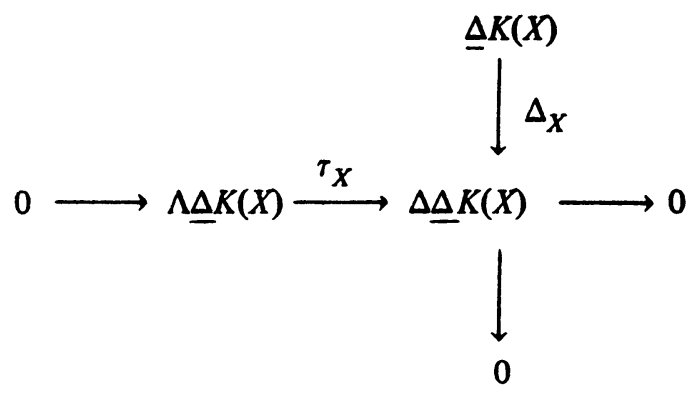

are exact and all mappings are continuous.

Proof. Let $X \in \mathcal{B}_{\omega}$ and suppose that $f \in K(X)$ and $\delta^{\circ}, f_{-t}$ is Bochner integrable. By Proposition 2.1, $K(X) \subseteq D_{2 n}$. Since $\delta^{\circ} f_{-t}$ is Bochner integrable, $\delta_{\imath}^{\circ} f_{t}$ is weakly integrable. Therefore for every $\varphi \in C_{2 n}^{\infty}$

$$
\frac{1}{(2 \pi)^{n}} \int \delta_{\imath}^{\circ} f_{-t} d t(\varphi)=f\left(\frac{1}{(2 \pi)^{n}} \int \delta_{t} \otimes \delta_{-t}(\varphi) d t\right)=f(\Delta \varphi)=\Delta_{X} f(\varphi) .
$$

Hence

$$
\begin{aligned}
\|\Delta f\|_{K(X)} & =\left\|\frac{1}{(2 \pi)^{n}} \int \delta^{\circ}, f_{-t} d t\right\|_{K(X)} \\
& \leq \frac{1}{(2 \pi)^{n}} \int\left\|\delta^{\circ}, f_{-t}\right\| d t=\|f\|_{\Delta K(X)} .
\end{aligned}
$$


Furthermore

$$
\begin{aligned}
\|\Delta f\|_{\Delta K(X)} & =\frac{1}{(2 \pi)^{n}} \int\left\|\delta_{\imath}^{\circ}(\Delta f)_{-t}\right\|_{K(X)} d t \\
& =\int\left\|\frac{1}{(2 \pi)^{n}} \int \delta_{s+t}^{\circ} f_{-s-t} d s\right\| d t=\|\Delta f\|_{K(X)} .
\end{aligned}
$$

Hence $\Delta: \Delta K(X) \rightarrow \Delta K(X)$ is continuous. The continuity of $\tau_{X}$ and the exactness of the sequences for Theorem 2.1 is obvious.

In the case of functors acting on the category $\mathcal{B}_{0}$ it is possible to obtain very much more extensive information.

Definition 2.4. Let $T^{n}$ be a toroidal group and $L^{\infty}$ the $L^{\infty}$ functor on $\mathcal{B}_{0}$. Suppose that $K$ is a norm subfunctor of $L^{\infty}$, between $C^{\infty}$ and $D_{n}$, and $K: \mathcal{B}_{0} \rightarrow \mathcal{M}_{0}$. Define functors $\Lambda K, \Delta K, \nabla K$ from $\mathcal{B}_{0}$ to $\alpha M_{0}$ by

$$
\begin{array}{rlrlrl}
\Lambda K(X) & =\Lambda_{X} K(X), & \Lambda K(\alpha) f=\Lambda_{X} K(\alpha) \tau_{X} f, & & \\
\|f\|_{\Lambda K(X)} & =\left\|\tau_{X} f\right\|_{K(X)}, & & \forall f \in \Lambda K(X) ; \\
\Delta K(X) & =\Delta_{X} K(X), \quad \Delta K(\alpha) f=\Delta_{X} K(\alpha) \Delta_{X} f, & & \\
\|f\|_{\Delta K(X)} & =\|f\|_{K(X)}, & & \forall f \in \Delta K(X) ; \\
\nabla K(X) & =\nabla_{X} K(X), \quad \nabla K(\alpha) f=\nabla_{X} K(\alpha) \nabla_{X} f, & \\
\|f\|_{\nabla K(X)} & =\|f\|_{K(X)}, & & \forall f \in \nabla K(X) .
\end{array}
$$

Using Proposition 2.1 it is also possible to define mappings $\Delta^{*}$ and $\Lambda^{*}$ for functors $K: \mathcal{B}_{0} \rightarrow \propto M_{0}$.

Definition 2.5. Let $T^{n}$ be a toroidal group and $L^{\infty}$ the $L^{\infty}$ functor on $T^{n}$; suppose that $K$ is a norm subfunctor of $L^{\infty}$, between $C_{n}^{\infty}$ and $D_{n}$, such that $K: \mathcal{B}_{0} \rightarrow \mathcal{M}_{0}$. For every $Y \in \mathcal{B}$ define mappings

$$
\begin{aligned}
\Delta^{*}: \operatorname{Hom}_{\circledast}[K, K] & \rightarrow \operatorname{Hom}_{\circledast}[\Delta K, \Delta K], \\
\Lambda^{*}: \operatorname{Hom}_{\otimes}[K, K] & \rightarrow \operatorname{Hom}_{\otimes}[\Lambda K, \Lambda K]
\end{aligned}
$$

by $\Delta_{Y}^{*}\left(H_{X}\right)=\Delta_{X \otimes Y} H_{X} \Delta_{X}, \Lambda_{Y}^{*}\left(H_{X}\right)=\Lambda_{X \otimes Y} H_{X} \tau_{X}$ for every $X \in \mathcal{B}_{0}$ and $H$ $\in \mathrm{Hom}_{\otimes}[K, K]$.

It is necessary to show that the functors (1), (2), (3) are well-defined functors in the category $\mathcal{B}_{0}$, in particular that the action of the functors on mappings $\alpha \in[X, Y]$ is well defined. Furthermore it is necessary to show that the mappings of Definition 2.5 have the indicated ranges. The first of these facts will come as a corollary to the next two theorems; the second will be proved as part of one of them (Theorem 2.3).

Theorem 2.2. Let $T^{n}$ be a toroidal group and $L^{\infty}$ the $L^{\infty}$ functor on $\mathcal{B}_{0}$. Suppose that $K$ is a norm subfunctor of $L^{\infty}$, between $C_{n}^{\infty}$ and $D_{n}$, and $K: \mathcal{B}_{0} \rightarrow \infty M_{0}$. Let 
$\Delta: D_{2 n} \rightarrow D_{2 n}$ be the mapping of Definition 2.1. Then for every $X \in \mathcal{B}_{0}, \Delta: K(X)$ $\rightarrow K(X)$.

Proof. I shall give the proof in a succession of lemmas.

Lemma 2.2.1. Let $X \in \mathcal{B}_{0}$ and suppose $X$ consists of distributions $\lambda: C^{\infty} \rightarrow B$ with values in a Banach space $B$; let $E_{X}=\left\{e_{m} \mid \exists \lambda \in X \ni \hat{\lambda}(m) \neq 0\right\}$ and $C_{X}^{\infty}=\mathrm{cl}_{C^{\infty}} \operatorname{Span} E_{X}$. Then there exists a closed subspace $Y \subseteq X^{*}$ such that

(1) $C_{X}^{\infty} \otimes B^{*}$ is dense in $Y$;

(2) $X$ is isomorphic up to equivalence of norms to a closed subspace of $Y^{*}$.

Proof. This lemma is simply a restatement of Definition 2.1 of the category $\mathfrak{B}_{0}$. Since $X \subseteq D_{n}(B), C_{X}^{\infty} \otimes B^{*}$ defines a class of continuous linear functionals on $X$ by

$$
\left\langle b^{*} \otimes \varphi, \lambda\right\rangle_{x}=\left\langle b^{*}, \lambda(\varphi)\right\rangle_{B}
$$

where (2.11) may be extended by linearity to all of $C_{X}^{\infty} \otimes B^{*}$.

Lemma 2.2.2. Let $L^{\infty}$ be the $L^{\infty}$ functor on $\mathcal{B}_{0}$ over $T^{n}$. Suppose that $X \in \mathcal{B}_{0}$ and $Y$ is the closed subspace of $X^{*}$ given by Lemma 2.2.1. Then for every $f \in L$, and $\lambda \in L^{\infty}(X)=\left[L^{1}, X\right]$

$$
\Delta \lambda(f)=\frac{1}{(2 \pi)^{n}} \int L^{\infty}\left(\delta_{t}\right) \lambda_{-t}(f) d t \in X
$$

where the integral in (2.11) exists in the weak sense on $Y$.

Furthermore the inequality

$$
\|\Delta \lambda\|_{L^{\infty}(X)} \leq\|\lambda\|_{L^{\infty}(X)}
$$

holds.

Proof. Since $\lambda: L^{1} \rightarrow X$ and $X \subseteq Y^{*}$ by Lemma 2.2.1, $\lambda: L^{1} \rightarrow Y^{*}$. Hence by the isomorphism of Proposition 1.3, $\lambda$ may be identified with the mapping $\lambda: L^{1} \otimes Y \rightarrow \mathrm{C}$. Since $C_{n}^{\infty} \subseteq L^{1}$ and $C_{X}^{\infty} \otimes B^{*} \subseteq Y, \lambda: C_{n}^{\infty} \otimes C_{X}^{\infty} \otimes B^{*} \rightarrow \mathbf{C}$.

Therefore for every $b^{*} \in B^{*}, f \in C_{n}^{\infty} \subseteq L^{1}$, and $\varphi \in C_{X}^{\infty}$,

$$
\begin{aligned}
\left\langle b^{*}, \Delta \lambda(f)(\varphi)\right\rangle_{B} & =\left\langle b^{*}, \Delta \lambda(f \otimes \varphi)\right\rangle_{B}=\left\langle b^{*}, \lambda(\Delta f \otimes \varphi)\right\rangle_{B} \\
& =\left\langle b^{*}, \lambda\left(\frac{1}{(2 \pi)^{n}} \int \delta_{-t} \otimes \delta_{t}(f \otimes \varphi) d t\right)\right\rangle \\
& =\left\langle b^{*}, \frac{1}{(2 \pi)^{n}} \int L^{\infty}\left(\delta_{k}\right) \lambda_{-t}(f \otimes \varphi) d t\right\rangle .
\end{aligned}
$$

Since (2.14) holds for all $\varphi \in C_{X}^{\infty}, f \in C_{n}^{\infty}$, and $b^{*} \in B^{*}$, and $C_{n}^{\infty} \otimes C_{X}^{\infty} \otimes B^{*}$ is dense in $L^{1} \otimes Y$,

$$
\Delta \lambda(f)=\frac{1}{(2 \pi)^{n}} \int L^{\infty}\left(\delta_{t}\right) \lambda_{-}(f) d t
$$


and $\Delta \lambda(f)$ exists as a weak integral with respect to $Y$ for every $f \in L^{\mathbf{L}}$.

I shall now show that $\Delta \lambda(f) \in X$ for every $f \in L^{1}$. Let $f \in L^{1}$ and observe that

$$
\Delta \lambda(f)=\frac{1}{(2 \pi)^{n}} \int L^{\infty}\left(\delta_{t}\right) \lambda_{-t}(f) d t=\frac{1}{(2 \pi)^{n}} \int L^{\infty}\left(\delta_{t}\right) \lambda\left(f_{t}\right) d t .
$$

Since $f_{t}$ is a continuous function of $t$ in $L^{1}$, and $\lambda: L^{1} \rightarrow X$ is a continuous operator, $\lambda\left(f_{t}\right)$ is a continuous function of $t$ in $X$. Therefore there exists a sequence of trigonometric polynomials $P_{N}(t)=\Sigma^{N} x_{m} e_{m}(t)$ with coefficients in $X$, and converging to $\lambda\left(f_{t}\right)$ uniformly in $t$. Furthermore for every $m \in Z^{n}$ and $x \in X$,

$$
\begin{gathered}
\left\langle b^{*} \otimes \varphi, \frac{1}{(2 \pi)^{n}} \int L^{\infty}\left(\delta_{t}\right) x e_{m}(t) d t\right\rangle=\left\langle b^{*}, x\left(\frac{1}{(2 \pi)^{n}} \int \varphi_{-t} e_{m}(t) d t\right)\right\rangle \\
=\left\langle b^{*}, x\left(\varphi * e_{m}\right)\right\rangle=\left\langle b^{*}, \hat{\varphi}(m) x\left(e_{m}\right) x\left(e_{m}\right)\right\rangle \\
=\left\langle b^{*}, \hat{x}(m)\right\rangle\left\langle\varphi, e_{m}\right\rangle=\left\langle b^{*} \otimes \varphi, \hat{x}(m) e_{m}\right\rangle .
\end{gathered}
$$

Therefore $(2 \pi)^{-n} \int L^{\infty}\left(\delta_{t}\right) x e_{m}(t) d t=\hat{x}(m) e_{m}$ where $\hat{x}(m) \in B$. Since $X \in \mathcal{B}_{0}$, it follows by condition (3) of Definition 1.18 that $X$ contains all elements of the form $b e_{m}$ for $b \in B$. Therefore $(2 \pi)^{-n} \int L^{\infty}\left(\delta_{t}\right) x e_{m}(t) d t \in X$.

Let $P_{N}(t)=\Sigma^{N} x_{m} e_{m}(t)$ be a trigonometric polynomial approximating to $\lambda\left(f_{t}\right)$. Since $Y$ is a subspace of $X^{*}$ norming for $X$,

$$
\begin{aligned}
\left\|\int L^{\infty}\left(\delta_{t}\right)\left[\lambda\left(f_{t}\right)-P_{N}(t)\right] d t\right\|_{X} & =\sup _{\|y\|_{x} \leq \leq 1}\left\langle y, \int L^{\infty}\left(\delta_{t}\right)\left[\lambda\left(f_{t}\right)-P_{N}(t)\right] d t\right\rangle \\
& \leq \int\left\|L^{\infty}\left(\delta_{t}\right)\left[\lambda\left(f_{t}\right)-P_{N}(t)\right]\right\|_{X} d t .
\end{aligned}
$$

Since $X \in \mathcal{B}_{0}$, the norm of $X$ is invariant with respect to translation. Therefore

$$
\left\|L^{\infty}\left(\delta_{t}\right)\left[\lambda\left(f_{t}\right)-P_{N}(t)\right]\right\|_{X}=\left\|\lambda\left(f_{t}\right)-P_{N}(t)\right\|_{X} .
$$

Since (2.15) is clearly measurable and $\rightarrow 0$ uniformly with respect to $t$,

$$
\left\|\int L^{\infty}\left(\delta_{t}\right)\left[\lambda\left(f_{t}\right)-P_{N}(t)\right] d t\right\|_{X} \rightarrow 0
$$

and $\Delta \lambda(f) \in X$ as the strong limit of elements of $X$.

To show the inequality $(2.13)$ it is now only necessary to observe that

$$
\|\Delta \lambda(f)\|_{X} \leq \frac{1}{(2 \pi)^{n}} \int\left\|L^{\infty}\left(f_{t}\right)\right\|_{X} d t \leq\|\lambda\|_{L^{\infty}(X)}\|f\|_{L^{\prime}}
$$

Again by the translation invariance of the norms in $X$ and $L$. Hence $\|\Delta \lambda\|_{L^{\infty}(X)}$ $\leq\|\lambda\|_{L^{\infty}(x)}$.

The theorem will be proved completely with the proof of the following lemma. 
Lemma 2.2.3. Let $K$ be a norm subfunctor of $L^{\infty}$, between $C^{\infty}$ and $D_{n}$, and $K: \mathbb{B}_{0} \rightarrow{ }{ }_{0}$. Suppose that $X \in \mathcal{B}_{0}$. Then $\|\Delta \lambda\|_{K(X)} \leq\|\lambda\|_{K(X)}$.

Proof. Let $X \in \mathcal{B}_{0}$ and suppose that $K$ is a norm subfunctor of $L^{\infty}$. Then the unit sphere $B_{1} K(X)=\left\{\lambda \in L^{\infty}(X) \mid\|\lambda\|_{K(X)} \leq 1\right\}$ is clearly a closed convex subset of $L^{\infty}(X)$ containing 0 .

Let $X$ consist of distributions with values in a Banach space $B$ and let $i_{X}$ be the isometric monomorphism

$$
\left[L^{1}, X\right] \stackrel{i x}{=}\left[L^{1} \otimes Y\right]^{*}
$$

defined by the composition of

$$
[L, X] \stackrel{J_{X}^{1}}{\subseteq}\left[L, Y^{*}\right] \stackrel{J_{X}^{2}}{\cong}\left[L^{1} \otimes Y\right]^{*}
$$

Since all mappings are isometries, $i_{X}$ is an isometry. Hence in particular the image $i_{X}\left(B_{1} K(X)\right)=S_{1}$ forms a closed convex subset of $(L \otimes Y)^{*}$ and $\|\cdot\|_{K(X)}$ isometrically generates a seminorm $\|\cdot\|_{K(X)}^{*}$ on $\left(L^{1} \otimes Y\right)^{*}$ by the Minkowski functional of $S_{1}$.

By the first part of Lemma 2.2.2, $L^{\infty}\left(\delta_{t}\right) \lambda_{-t}$ is weakly* integrable in $\left[L^{1} \otimes Y\right]^{*}$. Since $\left(S_{i}^{\circ}\right)^{\circ}=S_{1}$, it is sufficient to observe that for every $u \in S_{i}^{\circ}$,

$$
\left\langle u, i_{X}\left(\frac{1}{(2 \pi)^{n}} \int L^{\infty}\left(\delta_{t}\right) \lambda_{-t} d t\right)\right\rangle \leq \frac{1}{(2 \pi)^{n}} \int\left\|L^{\infty}\left(\delta_{t}\right) \lambda_{-t}\right\|_{K(X)}^{*} d t=\|\lambda\|_{K(X)}
$$

and hence $\|\Delta \lambda\|_{K(x)} \leq\|\lambda\|_{K(x)}$.

The next theorem follows immediately from the previous theorem by the use of Theorem 2.0 and Definition 2.2.

Theorem 2.3. Let $T^{n}$ be a toroidal group, $\mathcal{B}$ the category of Banach spaces, and $L^{\infty}$ the $L^{\infty}$ functor on $\mathcal{B}_{0}$. Then for every norm subfunctor $K$ of $L^{\infty}$ such that $K$ is between $C^{\infty}$ and $D_{n}$, and $K: \mathcal{B}_{0} \rightarrow \mathcal{M}_{0}$, and for every $X \in \mathcal{B}_{0}$ :

(1) If $J_{X}$ denotes the embedding map of $\nabla K(X)$ to $K(X)$, the sequence

$$
0 \rightarrow \nabla K(X) \stackrel{J_{x}}{\rightarrow} K(X) \stackrel{\Delta_{X}}{\rightarrow} \Delta K(X) \rightarrow 0 \text { is split exact. }
$$

(2) In the diagram

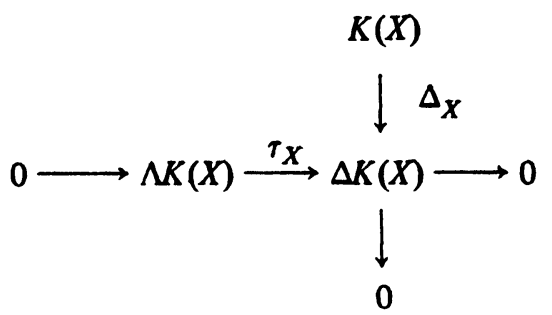

all sequences are exact. 
(3) For every $Y \rightarrow B$ the sequences

$$
\begin{aligned}
& 0 \rightarrow \operatorname{Hom}_{\otimes}[K, K](Y) \stackrel{\Lambda_{Y}^{*}}{\rightarrow} \operatorname{Hom}_{\circledast}[\Delta K, \Delta K](Y), \\
& 0 \rightarrow \operatorname{Hom}_{\otimes}[K, K](Y) \stackrel{\Lambda_{Y}^{*}}{\rightarrow} \operatorname{Hom}_{\circledast}[\Lambda K, \Lambda K](Y)
\end{aligned}
$$

are exact.

Proof. To see that (1) is true it is only necessary to note that the embedding map $J_{X}$ is well defined since by Theorem $2.2, \nabla K(X)=(I-\Delta) K(X) \subseteq K(X)$. (1) then follows from Theorem 2.0. (2) follows immediately from Theorem 2.0 since $\Delta K(X)$ is simply the image of $K(X) \subseteq D_{2 n}(B)$ under the mapping $\Delta$.

I shall now show part (3). By Theorem 2.7 below (Proposition 1.6) it follows that if $X \in \mathcal{B}_{0}$, then $X \times Y \in \mathcal{B}_{0}$ for any $Y \in \mathcal{B}$. Therefore $\Delta: K(X) \rightarrow K(X)$ and $\Delta: K(X \times Y) \rightarrow K(X \times Y)$ for every $X \in \mathcal{B}_{0}$ and $Y \in \mathcal{B}$. It is then clear from part (2) that the mappings of (3) have the indicated ranges. The rest of (3) follows immediately from Theorem 2.0 and the definitions (Definition 2.1, Definition 2.5) of $\Delta_{Y}^{*}$ and $\Lambda_{Y}^{*}$.

Remark. If $Y=\mathrm{C}$, the complex numbers, then the corresponding version of part (3) clearly follows without the use of Theorem 2.7. This fact will be important in the proof of Theorem 2.7 itself below.

Corollary 2.3.1. The functors (1), (2), (3) of Definition 2.4 are well defined for any functor $K: \mathbb{B}_{0} \rightarrow \mathcal{M}_{0}$ satisfying the conditions of Theorems 2.2 and 2.3.

Proof. To establish Corollary 2.3 .1 it is only necessary to show that the definitions of mappings in Definition 2.1 define mappings from $\Lambda K(X)$ to $\Lambda K(X)$ and similarly for other functors. For (2) and (3) this is clear from Theorem 2.2 alone; for (1) this is clear from Theorem 2.3(2) combined with Theorem 2.2.

It should be especially noted in the case of Theorem 2.3 that on the category $\mathcal{B}_{0}$ the mappings of (1), (2), (3) are not natural mappings. This is easy to see; general linear operators $\alpha \in[X, Y]$ will not commute with any of $\Lambda, \Delta$, or $\tau$.

However a category may be described on which the mappings $\Lambda, \Delta$, and $\tau$ of Theorem 2.3 will be natural. Let $\mathcal{M}_{0}^{\prime}$ be the category of all $X \in \mathcal{O} M_{0}$ such that $X \in \mathcal{B}_{0}$ also as a Banach space; the mappings of $\mathcal{M}_{0}^{\prime}$ will again be the set of all module homomorphisms $\alpha \in[X, Y]$. In the category $\delta M_{0}^{\prime}$ the mappings $\Delta, \Lambda, \tau$ of Theorem 2.3 will all be natural.

Theorem 2.4. Let $T^{n}$ be a toroidal group, $\mathcal{B}$ the category of Banach spaces, and $L^{\infty}$ the $L^{\infty}$ functor on $M_{0}^{\prime}$. Then for any norm subfunctor $K$ of $L$ satisfying the conditions of Theorems 2.2 and 2.3, all statements (1), (2), (3) of Theorem 2.3 hold for the functors $K, \Delta K, \Lambda K$, and all mappings are natural.

Proof. Since as a class of Banach spaces $\mathcal{M}_{0}^{\prime} \subseteq \mathcal{B}_{0}$, all statements of Theorem 2.3 hold for every $X \in M_{0}^{\prime}$. I will prove here only that the mapping $\Delta: K \rightarrow \Delta K$ 
is natural; the other cases are quite similar. To see that $\Delta$ is natural, observe that for every $X, Y \in M_{0}^{\prime}$, and $\alpha \in[X, Y], \alpha$ commutes with translation since $X$ and $Y$ are modules over $M_{0}$. Furthermore it will be sufficient to show naturalness only for $L^{\infty}$ since $K$ is a norm subfunctor of $L^{\infty}$. Let $X, Y \in M_{0}^{\prime}, \alpha \in[X, Y]$, and $\lambda \in L^{\infty}(X)$. To show that $\Delta: K \rightarrow \Delta K$ is natural, I must show that the diagram

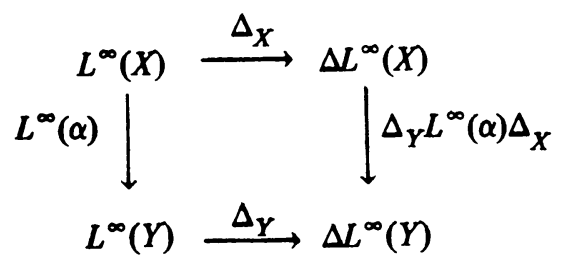

commutes, and hence that $\Delta_{Y} L^{\infty}(\alpha) \lambda=\Delta_{Y} L^{\infty}(\alpha) \Delta_{X}^{2} \lambda=\Delta_{Y} L^{\infty}(\alpha) \Delta_{X} \lambda$.

Since $\lambda \in L^{\infty}(X), \lambda \in D_{2 n}(B)$. For every $\varphi \otimes \psi \in C_{n}^{\infty} \otimes C_{n}^{\infty}=C_{2 n}^{\infty}$,

$$
L^{\infty}(\alpha) \Delta_{X} \lambda(\varphi \otimes \psi)=L^{\infty}(\alpha) \lambda(\Delta \varphi \otimes \psi)=\lambda\left(1_{C_{n}^{\infty}} \otimes \alpha \circ \Delta(\varphi \otimes \psi)\right) \equiv I .
$$

Since $\alpha$ is a convolution operator, $\alpha$ commutes with translation. Therefore

$$
\begin{aligned}
1_{C_{n}^{\infty}} \otimes \alpha(\Delta \varphi \otimes \psi) & =1_{C_{n}^{\infty}} \otimes \alpha\left(\frac{1}{(2 \pi)^{n}} \int \delta_{t} \otimes \delta_{-t}(\varphi \otimes \psi) d t\right) \\
& =\frac{1}{(2 \pi)^{n}} \int \delta_{t} \otimes \delta_{-t}(\varphi \otimes \alpha \psi) d t=\Delta\left(1_{C_{n}^{\infty}} \otimes \alpha\right)(\varphi \otimes \psi) .
\end{aligned}
$$

By (2.17) it then follows that

$$
I=\lambda\left(\Delta\left(l_{C_{n}^{\infty}} \otimes \alpha\right)(\varphi \otimes \psi)\right)=L^{\infty}(\alpha) \lambda(\Delta \varphi \otimes \psi)=\Delta_{Y} L^{\infty}(\alpha) \lambda(\varphi \otimes \psi)
$$

so that $L^{\infty}(\alpha) \Delta_{X} \lambda=\Delta_{Y} L^{\infty}(\alpha) \lambda$ for every $\lambda \in L^{\infty}(X)$ and $\alpha \in[X, Y]$. Hence $\Delta_{Y} L^{\infty}(\alpha) \lambda=\Delta_{Y} L^{\infty}(\alpha) \Delta_{X}^{2} \lambda=\Delta_{Y} L^{\infty}(\alpha) \Delta_{X} \lambda=\Delta_{Y}^{2} L^{\infty}(\alpha) \lambda=\Delta_{Y} L^{\infty}(\alpha) \lambda \quad$ which shows the commutativity of the diagram (2.16).

In the applications of Theorem 2.3 I shall often deal with spaces of the forms $K(X)$ or $K(X \otimes Y)$ where $X \in \mathcal{B}_{0}, Y \in \mathcal{B}$, and $K$ is a translation invariant norm subfunctor of $L^{\infty}$. The next theorem will frequently be useful in providing alternative descriptions of such spaces.

Definition 2.6. Let $\mu: R^{+} \rightarrow R^{+}$be a positive nonincreasing function continuous on the left, and suppose that $L^{\infty}$ is the $L^{\infty}$ functor on the category $\mathcal{B}$. Define

$$
\begin{aligned}
C^{\mu}(X) & =\left\{\lambda \in L^{\infty}(X) \mid \sup _{s} \mu(s)\left\|\lambda_{s}-\lambda\right\|_{L^{\infty}(X)}<\infty\right\}, \\
\|\lambda\|_{C^{\mu}(X)} & =\|\lambda\|_{L^{\infty}(X)}+\sup _{s} \mu(s)\left\|\lambda_{s}-\lambda\right\|_{L^{\infty}(X)}, \\
C^{\mu}(\alpha) \lambda & =L^{\infty}(\alpha) \lambda .
\end{aligned}
$$


It is clear that for every $\mu, C^{\mu}$ is a norm subfunctor of $L^{\infty}$. A functor of the type of Definition 2.6 shall be referred to as a continuity functor.

Theorem 2.5. Let $T^{n}$ be a toroidal group, $L^{\infty}$ the $L^{\infty}$ functor on the category $\mathcal{B}_{0}$ over $T^{n}$. Then:

(1) For every continuity function $\mu$ and $X \in \mathcal{B}_{0}, \Lambda C^{\mu}(X) \subseteq \Lambda C(X)$.

(2) For every $X \in \mathcal{B}_{0}, \Lambda C(X) \subseteq X \subseteq \Lambda L^{\infty}(X)=W(X)$, where each space is a closed subspace of the following space.

(3) For every $X \in \mathcal{B}_{0}$ and $Y \in \mathcal{B}, \Lambda L^{\infty}(X \otimes Y)=\Lambda L^{\infty}(X) \otimes Y$.

Proof. (1) follows immediately from the definitions of $\Lambda, C$, and $C^{\mu}$. I shall prove (2) from which (3) will also follow immediately.

I shall first show that $\Lambda C(X) \subseteq X$. Since $X \in \mathcal{B}_{0}, X$ is harmonically invariant. Hence there exists a norming subspace $V\left(X^{*}\right)$ of $X^{*}, V\left(X^{*}\right)=\operatorname{cl}_{X^{*}} C_{X}^{\infty} \otimes B^{*}$. Suppose $\lambda \in \Lambda C(X)$. Then by Theorem 2.3, $\tau(\lambda) \in C(X)$. Since $X \subseteq D_{n}(B)$ for some Banach space $B$, by Theorem 2.3 again and the definition of the mapping $\tau$, for every $f \in L^{\prime}$ and $\varphi \in C_{X}^{\infty} \otimes B^{*}$,

$$
\langle\tau(\lambda)(f), \varphi\rangle=\langle\tau(\lambda), f \otimes \varphi\rangle=\left\langle\lambda, \int \varphi_{-t} f(t) d t\right\rangle=\left\langle\int \lambda_{t} f(t) d t, \varphi\right\rangle
$$

where the integral in (2.18) is taken in the weak ${ }^{*}$ sense in $W(X)=V\left(X^{*}\right)^{*}$. Hence $\tau(\lambda)(f)=\int \lambda_{t} f(t) d t$.

By the Dunford-Pettis theorem [10, VI, 8.6], there exists a unique $W(X)$-valued function $\tau(\lambda)_{0}$ such that

$$
\tau(\lambda)(f)=\int \tau(\lambda)_{0}(t) f(t) d t
$$

Hence $\tau(\lambda)_{0}(f)=\lambda_{t}$ a.e. Furthermore $\|\tau(\lambda)\|_{C(X)}=\sup _{s}\left\|\lambda_{s}\right\|_{W(X)}=\|\lambda\|_{W(X)}$ where the last equality holds because of the translation invariance of the $W(X)$ norm. Since $\tau(\lambda) \in C(X), \lambda_{s}$ is a continuous $W(X)$-valued function of $s$. Let $p_{k}$ be an approximate identity in $L^{\prime}$ consisting of trigonometric polynomials. Then since $\lambda_{t}$ is a continuous function of $t$ and $X$ is a closed subspace of $W(X)$ (by the Definition 1.18 of harmonic invariance) it follows that $\tau(\lambda)\left(p_{k}\right)=\int \lambda_{t} p_{k}(t) d t$ converges strongly in $W(X)$ to $\lambda$. However for every $e_{m}$ and $y \in V\left(X^{*}\right)$,

$$
\begin{aligned}
\left\langle y, \int \lambda_{t} e_{m}(t) d t\right\rangle & =\left\langle\int y_{-t} e_{m}(t) d t, \lambda\right\rangle \\
& =\left\langle\hat{y}(m) e_{m}, \lambda\right\rangle=\left\langle y, \hat{\lambda}(m) e_{m}\right\rangle .
\end{aligned}
$$

Since $X$ is harmonically invariant, $\hat{\lambda}(m) e_{m} \in X$ for every $m$; therefore $\tau(\lambda)\left(p_{k}\right)$ $=\int \lambda_{t} p_{k}(t) d t \in X$ for every $p_{k}$. Since $X$ is closed it follows that $\lambda \in X$ and thus $\Lambda C(X) \subseteq X$.

I shall now show that $W(X)=\Lambda L^{\infty}(X)$. Since $X$ is a closed subspace of $W(X),(2)$ will follow. Suppose then that $\lambda \in W(X)=V\left(X^{*}\right)^{*}$. Then the weak integral $\tau(\lambda)(f)=\int \lambda_{t} f(t) d t$ exists in $W(X)$. $\tau(\lambda)$ therefore defines a continuous 
function $\tau(\lambda): L^{\prime} \rightarrow W(X)$ for every $\lambda \in W(X)$. Let $f \in L^{1}$. Then there exists a sequence of trigonometric polynomials $\left\langle p_{k}\right\rangle, p_{k} \rightarrow f$ in $L$. Furthermore, by (2.19), $\tau(\lambda)\left(p_{k}\right)=\int \lambda_{t} p_{k}(t) d t \in X$ for every $k$. Since $p_{k} \rightarrow f$ in $L$, $\tau(\lambda)\left(p_{k}\right)$ $\rightarrow \tau(\lambda)(f)$ and therefore $\tau(\lambda)(f) \in X$ for every $f \in L^{1}$. Hence $\tau(\lambda): L^{L} \rightarrow X$ and $\lambda \in \Lambda L^{\infty}(X)$ for every $\lambda \in W(X)$. Thus $W(X) \subseteq L^{\infty}(X)$.

To show that $\Lambda L^{\infty}(X) \subseteq W(X)$, I shall first prove a lemma.

Lemma 2.5.1. Let $f$ be a complex-valued measurable function of $T^{n}$. Then the function $f(x, y)=f(x+y)$ for $(x, y) \in T^{2 n}$ is a measurable function on $T^{2 n}$.

Proof. Clearly the case of complex-valued functions may be reduced to that of real-valued functions; I shall suppose $f$ has real values. $f$ is measurable if and only if $\{x \mid f(x)>\alpha\}$ is measurable for every $\alpha$. Let $E_{\alpha}=\{x \mid f(x)>\alpha\}$ and consider the set $F_{\alpha}=\{(x, y) \mid f(x+y)>\alpha\}$. $(x, y) \in F_{\alpha}$ iff $(x, y) \in U_{x \in T^{n}}\left(x, E_{\alpha}-x\right)$. Let $S: T^{2 n} \rightarrow T^{2 n}$ be the group homomorphism defined by

$$
\left[\begin{array}{ll}
1 & 1 \\
0 & 1
\end{array}\right][x, y]=[x, x+y] .
$$

Then $S\left[F_{\alpha}\right]=S\left(\cup_{x}\left(x, E_{\alpha}-x\right)\right)=\cup_{x}\left(x, E_{\alpha}\right)=E_{\alpha} \times T^{n}$. Let $\mu$ be the ordinary measure on $T^{2 n}$ and $\mu_{S}$ the measure on $T^{2 n}$ induced by $S^{-1}$ from $T^{2 n}$. Since Haar measure is unique and $E_{\alpha} \times T^{n}$ is measurable, it follows that $F=S^{-1}\left(E_{\alpha} \times T^{n}\right)$ is measurable and hence $\bar{f}$ is measurable.

To show that $\Lambda L^{\infty}(X) \subseteq W(X)$, let $\lambda$ be a distribution, $\lambda \in \Lambda L^{\infty}(X)$. Then $\tau(\lambda) \in L^{\infty}(X)=\left[L^{1}, X\right]$. Let $\left\langle h_{k}\right\rangle$ be a $C^{\infty}$ approximate identity in $L^{1}$ and define a distribution $n$ in $D_{n}\left(B^{* *}\right)$ by

$$
\left\langle\phi \otimes b^{*}, w\right\rangle=\lim _{k \rightarrow \infty}\left\langle\phi \otimes b^{*}, \tau(\lambda)\left(h_{k}\right)\right\rangle .
$$

I shall show that (2.20) defines a linear functional on $V\left(X^{*}\right)=\mathrm{cl}_{X} \cdot C_{X}^{\infty} \otimes B^{*}$ and hence an element of $W(X)$. Observe first that $\tau(\lambda)\left(h_{k}\right)$ defines a uniformly bounded sequence of elements of $X$ since $\left\|h_{k}\right\|=1 \forall k$ and $\tau(\lambda)$ is a continuous mapping $\tau(\lambda): L^{1} \rightarrow X$. Furthermore by Definition 2.1 of the mapping $\tau$ it follows that for every $\phi$ and $b^{*}$,

$$
\begin{aligned}
\left\langle\phi \otimes b^{*}, \tau(\lambda)\left(h_{k}\right)\right\rangle & =\left\langle h_{k} \otimes \phi \otimes b^{*}, \tau(\lambda)\right\rangle \\
& =\left\langle\left(h_{k} * \phi\right) \otimes b^{*}, \lambda\right\rangle \rightarrow\left\langle\phi \otimes b^{*}, \lambda\right\rangle .
\end{aligned}
$$

Since $C_{X}^{\infty} \otimes B^{*}$ is strongly dense in $V\left(X^{*}\right)$ it follows that $(2.20)$ defines a continuous linear functional on $V\left(X^{*}\right)$ and hence a member of $W(X)$. To show that $w=\lambda$ it is only necessary to observe that since $\Lambda: C_{2 n}^{\infty} \rightarrow C_{n}^{\infty}$,

$$
\begin{aligned}
\left\langle\phi \otimes b^{*}, \tau(w)(\psi)\right\rangle & =\left\langle\Lambda(\phi \otimes \psi) \otimes b^{*}, w\right\rangle \\
& =\left\langle\Lambda(\phi \otimes \psi) \otimes b^{*}, \lambda\right\rangle=\left\langle\phi \otimes b^{*}, \tau \lambda(\psi)\right\rangle .
\end{aligned}
$$


Since $C_{n}^{\infty}$ is dense in $L^{1}$ and $\tau$ is a monomorphism it follows that $\lambda=w$. Hence $\Lambda L^{\infty}(X) \subseteq W(X)$. By the previous argument, $W(X) \subseteq \Lambda L^{\infty}(X)$ and thus equality holds.

To show part (3), observe first that for every $X \in \mathcal{B}_{0}$ and $v \in V\left(X^{*}\right)$, the translation operator $\delta_{s} v$ defines a strongly continuous mapping from $T^{n}$ to $V\left(X^{*}\right)$. Hence it follows that, for every $\lambda \in X,\left\langle v, \delta_{s} \lambda\right\rangle=\left\langle\delta_{-s} v, \lambda\right\rangle$ is a continuous function of $s$ for every $\lambda \in X$, and thus $\delta_{s} \lambda$ is a weakly measurable function of $s$. Therefore the weak integral $\bar{\tau}(\lambda)(f)=\int \lambda_{s} f(s) d s$ is a well-defined weak ${ }^{*}$ integral for every $f \in L^{1}$, and for every $\lambda \in W(X)=\Lambda L^{\infty}(X)$ will define a mapping $\bar{\tau}(\lambda): L^{1} \rightarrow W(X)$.

Let $\phi, \psi \in C_{n}^{\infty}$ and $b^{*} \in B^{*}$. Then

$$
\begin{aligned}
\left\langle\phi \otimes b^{*}, \bar{\tau}(\lambda)(\psi)\right\rangle & =\left\langle\phi \otimes b^{*}, \int \lambda_{s} \psi(s) d s\right\rangle \\
& =\left\langle(\phi * \psi) \otimes b^{*}, \lambda\right\rangle=\left\langle\phi \otimes b^{*}, \tau(\lambda)(\psi)\right\rangle .
\end{aligned}
$$

Since $C_{n}^{\infty}$ is dense in $L^{1},(2.21)$ implies that $\tau(\lambda)=\bar{\tau}(\lambda)$.

Now observe that for every $Y \in \mathcal{B}$ there exists a natural embedding $\Sigma_{Y} \circ L^{\infty}$ $\subseteq L^{\infty} \circ \Sigma_{Y}$ defined by

$$
i_{x}[L \otimes y](f)=L(f) \otimes y, \quad f \in L^{1},
$$

and extended by linearity for every element of $L^{\infty}(X) \otimes Y$. Furthermore, for every $\lambda \in X$ and $y \in Y$,

$$
\bar{\tau}(\lambda) \otimes y(f)=\left(\int \lambda_{s} f(s) d s\right) \otimes y=\int\left(\lambda_{s} \otimes y\right) f(s) d s=\bar{\tau}(\lambda \otimes y)(f) .
$$

Hence for every $u \in \tau X \otimes Y, u=\Sigma^{\infty} \tau f^{k} \otimes y_{k}=\tau\left(\Sigma^{\infty} f^{k} \otimes y_{k}\right) \in \tau(X \otimes Y)$ and the reverse. Since $X \in \mathcal{B},\left\|f_{s}\right\|_{X}=\|f\|_{X}$. Hence, for every $u \in \tau(X \times Y)$,

$$
\begin{aligned}
\|\tau u\|_{L^{\infty}(X \otimes Y)} & =\sup _{s} \inf \sum\left\|f_{s}^{k}\right\|\left\|y_{k}\right\|=\inf \Sigma\left\|f^{k}\right\|\left\|y_{k}\right\| \\
& =\inf \sum\left(\sup _{s}\left\|f_{s}^{k}\right\|\right)\left\|y_{k}\right\|=\|\tau u\|_{L^{\infty}(X) \otimes Y}
\end{aligned}
$$

where the inf in (2.22) is taken over all representations $u=\sum f^{k} \otimes y_{k}$ of $u$. Thus the norms of $L^{\infty}(X) \otimes Y$ and $L^{\infty}(X \otimes Y)$ are identical when restricted to $\tau X \otimes Y=\tau(X \otimes Y)$. Part (3) is proved.

A stronger theorem may be proved for the category $M_{0}^{\prime}$.

Theorem 2.6. Let $T^{n}$ be a toroidal group, $L^{\infty}$ the $L^{\infty}$ functor, $C$ the continuity functor, and I the identity functor on the category o $M_{0}^{\prime}$. Then:

(1) For every continuity function $\mu$, there exists a natural embedding $\nu_{0}$,

$$
\Lambda C^{\mu} \stackrel{\nu_{0}}{\subseteq} \Lambda C
$$


(2) There exist natural embeddings $\nu_{1}$ and $\nu_{2}$, and an isomorphism isuch that

$$
\Lambda C \stackrel{p_{1}}{\subseteq} \stackrel{p_{2}}{\subseteq} \Lambda L^{\infty} \cong W
$$

where each functor is a closed subfunctor of the following functor.

(3) For every $Y \in \mathcal{B}, \Lambda L^{\infty} \circ \Sigma_{Y} \cong \Sigma_{Y} \circ \Lambda L^{\infty}$.

Proof. In each case the embeddings and isomorphisms are the identity mapping. The naturalness of all mappings follows from Theorem 2.4.

The next theorem states the basic fact concerning the functor $E_{Y}$ acting on the category $\mathcal{B}_{0}$. It was alluded to previously as Proposition 1.7.

Theorem 2.7. Let $Y \in \mathcal{B}$. Then $\Sigma_{Y}: \mathcal{B}_{0} \rightarrow \mathcal{B}_{0}$.

Proof. It is clear from the definition of $\mathcal{B}_{0}$ that if $u \in X \otimes Y$ then by Corollary 4.4.2 below and the action of the functor $\Sigma_{Y}$ on mappings:

$$
\|h * u\|_{X \otimes Y}=\inf \Sigma\left\|h * x_{k}\right\|\left\|y_{k}\right\| \leq\|h\|_{I}\|u\|_{X \otimes Y}
$$

and $X \otimes Y$ has translation invariant norm. Therefore $X \otimes Y$ is a module over $M_{0}$. Furthermore since $B E_{X} \subseteq X$ it follows also that $B \otimes Y E_{X} \subseteq X \otimes Y$.

I shall now show that $C_{X}^{\infty} \otimes[B \otimes Y]^{*}$ is a norming subspace of $X^{*}$.

First observe that if $X \in \mathcal{B}_{0}$, then $X \otimes Y \in M_{0}$. To show this, I must show that $X \otimes Y$ is normal. Let $\left\langle h_{n}\right\rangle$ be an approximate identity in $L$. Then, for every $x \in X$,

$$
\left\|h_{n} * x\right\|_{X}=\sup _{v \in V\left(X^{*}\right) ;\|v\| \leq 1}\left\langle v, h_{n} * x\right\rangle=\sup _{\|v\| \leq 1}\left\langle v * h_{n}, x\right\rangle \rightarrow\|x\| .
$$

Therefore, for every $u \in X \otimes Y$,

$$
\left\|h_{n} * u\right\|_{X \otimes Y}=\inf \Sigma\left\|h_{n} * x_{k}\right\|\left\|y_{k}\right\| \rightarrow \inf \sum\left\|x_{k}\right\|\left\|y_{k}\right\|=\|u\|_{X \otimes Y} .
$$

Hence if $X \in \mathcal{B}_{0}, X \otimes Y \in \mathcal{O}$.

Let $u^{*} \in(X \otimes Y)^{*}$ and define for every $e_{m} \in E_{X}=E_{X \otimes Y}, u_{m}^{*} \in[B \otimes Y]^{*}$ by $\left\langle u_{m}^{*}, b \otimes y\right\rangle=\left\langle u^{*}, b \otimes y e\right\rangle$. Since $B \otimes Y e_{m}$ is continuously contained in $X \otimes Y$, $u_{m}^{*}$ is well defined after extension by linearity. Furthermore $u_{m}^{*} \otimes e_{m} \in C_{X}^{\infty}$ $\otimes[B \otimes Y]^{*}$ and, for every $e_{m} \in E_{X}$,

$$
\begin{aligned}
\left\langle u^{*} * e_{m}, u\right\rangle & =\left\langle u^{*}, e_{m} * x\right\rangle=\left\langle u^{*}, u\left(e_{m}\right) e_{m}\right\rangle \\
& =\left\langle u_{m}^{*}, u\left(e_{m}\right)\right\rangle=\left\langle u_{m}^{*} \otimes e_{m}, u\right\rangle .
\end{aligned}
$$

Therefore $u^{*} * h \in \operatorname{cl}_{(X \otimes Y)^{*}} C_{X}^{\infty} \otimes[B \otimes Y]^{*}$ for every $h \in L^{1}$ and $u^{*} \in$ $(X \otimes Y)^{*}$. Let $V_{1}=\operatorname{cl}_{(X \otimes Y)^{*}} C_{X}^{\infty} \otimes[B \otimes Y]^{*}$. Then

$$
\begin{aligned}
\sup _{\| v k_{1} \leq 1}\langle v, u\rangle & =\sup _{\left\|h \cdot u^{*}\right\| \leq 1}\left\langle u^{*} * h, u\right\rangle=\sup _{\|h\|_{0} \leq 1} \sup _{\left\|u^{*}\right\| \leq 1}\left\langle u^{*}, h * u\right\rangle \\
& =\sup _{\|h\|_{b} \leq 1}\|h * u\|=\|u\|_{X \otimes Y} .
\end{aligned}
$$


Thus $C_{X}^{\infty} \otimes[B \otimes Y]^{*}$ is norming and $X \otimes Y \in \mathcal{B}_{0}$.

\section{CHAPTER 3: APPROXIMATE IDENTITIES OF CONVOLUTION OPERATORS}

In this section I shall give a treatment in terms of category theory of the convergence of sequence of convolution kernels of types which include among others the Fourier series and its means. The advantage of the notion of functor here derives from the fact that the scope of theorems on pointwise convergence is displayed much more clearly in this setting: the most noteworthy advantage of such an approach is the demonstration, as mentioned in the introduction, that the standard proofs of pointwise convergence extend easily to theorems on norm convergence of the convolution kernels in a very wide class of spaces of distributions.

All of the results of this chapter will be formulated for the group $T^{1}=R / 2 \pi Z$. However it should be observed (see V. Shapiro [39] and H. Shapiro [38]) that results on convergence and inverse theorems will extend to groups $T^{n}$.

1. Convergence of sequences of convolution kernels. I shall first define the functors and mappings of functors in terms of which theorems on convergence may be stated.

Definition 3.0. Let $\mathcal{B}$ be the category of Banach spaces and $Z^{+}$the set of all nonnegative integers. Define a functor $c_{0}$ on $\mathcal{B}$ by

$$
c_{0}(X)=\left\{\left\langle x_{n}\right\rangle: Z^{+} \rightarrow X \mid x_{n} \rightarrow 0 \text { as } n \rightarrow \infty\right\}
$$

the norm on $c_{0}(X)$ is defined as $\left\|\left\langle x_{n}\right\rangle\right\|_{c_{0}(X)}=\sup _{h}\left\|x_{n}\right\|_{X}$. The action of $c_{0}$ on mappings $\alpha \in[X, Y]$ for $X, Y \in \mathcal{B}$ is obtained:

$$
c_{0}(\alpha)\left(\left\langle x_{n}\right\rangle\right)=\left\langle\alpha\left(x_{n}\right)\right\rangle .
$$

If $X$ is a Banach module over a ring $R, c_{0}(X)$ may clearly be considered as a Banach module over $R$ with module multiplication for every $r \in[X, X]$ defined by (3.0). It is clear also that for every $X, c_{0}(X)$ is the space of sequences $\left\langle x_{n}\right\rangle \in X$ such that $x_{n} \rightarrow 0$ as $n \rightarrow \infty$.

Definition 3.1. Let $F_{1}, F_{2}$ be Banach space functors $F_{1}, F_{2}: \mathcal{B} \rightarrow \mathcal{B}, \sigma_{n}$ a sequence of natural mappings $\sigma_{n}: F_{1} \rightarrow F_{2}$, and $\sigma: F_{1} \rightarrow F_{2}$ a fixed natural mapping. Then I shall say $\sigma_{n}$ converges to $\sigma$ if the sequence $\Sigma=\langle\Sigma(n)\rangle$ defined for every $n \in Z^{+}$by $\Sigma(n)=\sigma_{n}-\sigma$ is a natural mapping $\Sigma: F_{1} \rightarrow c_{0} F_{2}$.

The first theorem holds for a very wide class of convolution kernels acting on the functor $C$ over $T^{1}$. Let $C$ be the continuity functor over $T^{1}$. Then clearly $C$ has a unique natural extension by periodicity to a functor $\bar{C}$ over the real line $\mathbf{R}$. In the following I shall consider $C(X)$ interchangeably as a space of continuous functions on $T^{1}$ and continuous $2 \pi$-periodic functions on $\mathbf{R}$. 
For every $h \in L$, complex-valued and integrable over $(-\infty, \infty)$, let $h_{n}$ be the function defined by $h_{n}(x)=n h(n x)$. Clearly $\left\|h_{n}\right\|_{1}=\|h\|_{1}$ and $h_{n} \in L^{1}$ for every $n$.

Definition 3.2. Let $B$ be the category of Banach spaces and $C$ the periodic continuity functor on $\mathbf{R}$. For every $h \in L^{1}, X \in \mathcal{B}$, and $f \in C(X)$ define

$$
\Sigma_{X}(h)_{n}(f)=f * h_{n}=\int_{-\infty}^{\infty} f(x-t) n h(n t) d t .
$$

For every $h, \Sigma_{X}(h)=\left\langle\Sigma_{X}(h)_{n}\right\rangle$ is clearly a sequence of mappings on $C(X)$. It is also clear from the representation (3.1) and the fact that convolution is natural, that $\Sigma(h)=\left\{\Sigma_{X}(h)_{n}\right\}$ is a natural mapping $\Sigma(h)_{n}: C \rightarrow C$ for every $n$.

Theorem 3.0. Let $\mathcal{B}$ be the category of Banach spaces and $I$ the identity mapping on the functor $C$. Then, for every $h \in L^{1}$ such that $\int h d t=1, \Sigma(h)$ converges to $I$.

Proof. This proof is quite standard; the difference from previous proofs consists only of the fact that it is done for vector-valued integrals. Let $X \in \mathcal{B}$ and $f \in C(X)$. Then

$$
\begin{aligned}
\Sigma_{X}(h)_{n}(f) & =I(f)=\int_{-\infty}^{\infty} f(x-t) n h(n t) d t-f \\
& =\int_{-\infty}^{\infty}\left[f\left(x-\frac{t}{n}\right)-f(x)\right] h(t) d t .
\end{aligned}
$$

It is then easy to see that $\Sigma_{X}(h)_{n}(f)-I(f) \rightarrow 0$ and thus the theorem is proved.

The following corollary follows immediately by Theorems 2.0 and 2.1 .

Corollary 3.0.1. Let $\mathcal{B}_{\omega}$ be the category of Banach distribution spaces on $T^{1}, C$ the continuity functor on $\mathcal{B}_{\omega}, I$ the identity mapping on $C$, and $\Delta C$ the functor of Definition 2.1. Then for every $h \in L^{1}$ such that (1) $h \geq 0$, (2) $\|h\|_{h}=1, \Sigma(h)$ converges to $I$ on $\underline{\Delta C}$ and hence for every $X \in \mathcal{B}_{\omega}$ and $f \in \Lambda \Delta C(X), h_{n} * f \rightarrow f$ in $\boldsymbol{X}$.

Proof. Clearly for every $X \in \mathcal{B}_{\omega}, \Lambda \Delta C(X)$ is identical to the class of $f \in X$ such that $\tau f$ is a continuous $X$-valued function. Corollary 3.0.1 follows immediately after restricting $\Sigma_{X}(h)$ to $\Delta C(X) \cong \Lambda \Delta C(X)$. To see that $h_{n} * f \rightarrow f$ in $X$ for every $f \in \Lambda C(X)$, observe that, for $f \in \Lambda C(X)$,

$$
\Sigma_{X}(h)_{n}(f)(0)=(\tau f) * h_{n}(0)=\int f_{-t} h_{n}(t) d t=f * h_{n} .
$$

Since $\Sigma_{X}\left(h_{n}\right)(f) \rightarrow \tau f$ uniformly, it follows that $\Sigma_{X}(h)_{n}(f)(0) \rightarrow \tau f(0)=f$ in $X$.

For a more restricted class of kernels it is possible to prove a very much stronger theorem.

Definition 3.3. Let $\mathcal{B}$ be the category of Banach spaces and $L^{1}$ the $L^{1}$ functor on $\mathcal{B}$ over $T^{1}$. For every $x \in T^{1}$ and $X \in \mathcal{B}$, define a function $\rho(x, f)$ from $L^{1}(X)$ to the set of all $x$-valued sequences by 


$$
\rho(x)(f)(n)=\frac{n}{2} \int_{-1 / n}^{1 / n}\|f(t-x)-f(x)\|_{x} d t .
$$

It is proved in Dunford and Schwartz [10, III.12.8] that for every $f \in L^{1}(X)$ and almost every $x \in T^{1}, \rho(x)(f)(n) \rightarrow 0$ as $n \rightarrow \infty$.

I shall now define the main functor in terms of which the next convergence results may be formulated.

Definition 3.4. Let $B$ be the category of Banach spaces and $L^{1}$ the $L^{1}$ functor defined on $\mathcal{B}$ over the group $T^{1}$. Let $x_{0} \in T^{1}$ and define a functor $L_{x_{0}}^{1}$ on $\mathcal{B}$ by

$$
\begin{aligned}
L_{x_{0}}^{1}(X) & =\left\{f \in L^{1}(X) \mid \rho\left(x_{0}\right)(f) \in c_{0}(X)\right\}, \\
\|f\|_{L_{0}}(X) & =\|f\|_{L^{\prime}}(X)+\left\|\rho\left(x_{0}\right)\right\|_{0_{0}}, \\
L_{x_{0}}^{1}(\alpha)(f)(t) & =\alpha f(t) .
\end{aligned}
$$

$L_{x_{0}}$ clearly defines a functor on $\mathcal{B}$. The results on convergence of convolution kernels will be formulated in terms of natural mappings from the functors $L_{x_{0}}^{1}$ to I.

Let $f: T^{1} \rightarrow X$ be an $X$-valued function defined and Bochner integrable on $T^{1}$ and define, for $n \in Z^{+}$and $x \in T^{1}$,

$$
\Sigma_{X}\left(h, x_{0}\right)_{n}(f)=\Sigma_{X}(h)_{n}(f)\left(x_{0}\right)=f * h_{n}\left(x_{0}\right) .
$$

Clearly for every $x_{0}, h, f$, and $n \in Z^{+}, \Sigma_{X}\left(h, x_{0}\right)_{n}(f)$ is a uniquely defined element of $X$; thus $\Sigma_{X}\left(h, x_{0}\right)(f)=\left\langle\Sigma_{X}\left(h, x_{0}\right)_{n}(f)\right\rangle$ defines a sequence in $X$.

It is now possible to prove the following theorem.

Theorem 3.1. Let $\mathcal{B}$ be the category of Banach spaces, let $x_{0} \in T^{1}$, and $\delta_{x_{0}}$ be the evaluation mapping at $x_{0}$ for the functor $L_{x_{0}}^{1}$. Then for every $h \in L^{\infty}$ such that $(1) h$ is even, (2) $\int h d t=1$, (3) for some $\alpha>1$ and $x$ large $h(x) \sim O\left(x^{\alpha}\right), \Sigma\left(h, x_{0}\right)$ converges to $\delta_{x_{0}}$ on $L_{x_{0}}^{1}$.

Proof. Let $\phi_{x_{0}}(f, t)=\frac{1}{2} f\left(x_{0}+t\right)+\frac{1}{2} f\left(x_{0}-t\right)-f\left(x_{0}\right)$. Then

$$
\begin{aligned}
\Sigma\left(h, x_{0}\right)_{n}(f)-f\left(x_{0}\right) & =\int_{-\infty}^{\infty} f\left(x_{0}-t\right) h_{n}(t) d t-f\left(x_{0}\right) \\
& =\int_{0}^{\infty} \phi_{x_{0}}(f, t) n h(n t) d t
\end{aligned}
$$

where $\phi_{x_{0}}(f, t)$ is integrable in $t$ since $f$ is periodic in $t$ and the integral (3.3) exists, $h_{n}$ is uniformly bounded for every $n$.

Furthermore

$$
\begin{aligned}
\int \phi_{x_{0}}(f, t) n h(n t) d t & =\int_{0}^{1 / n} \phi_{x_{0}}(f, t) n h(n t) d t+\int_{1 / n}^{\infty} \phi_{x_{0}}(f, t) n h(n t) d t \\
& =I_{1}(n, h)+I_{2}(n, h) .
\end{aligned}
$$


Since $h$ is bounded it follows that

$$
\begin{aligned}
\left\|I_{1}(n, h)\right\|_{X} & =\left\|\int_{0}^{1 / n} \phi_{x_{0}}(f, t) n h(n t) d t\right\|_{X} \leq B n \int_{0}^{1 / n}\left\|\phi_{x_{0}}(f, t)\right\|_{X} d t \\
& =B \rho\left(x_{0}, f\right)(n)
\end{aligned}
$$

where $B$ depends only on $h$. Again

$$
\begin{aligned}
I_{2}(n, h) & =\int_{1 / n}^{A} \phi_{x_{0}}(f, t) n h(n t) d t+\int_{A}^{\infty} \phi_{x_{0}}(f, t) n h(n t) d t \\
& =I_{2}^{\prime}(h, n)+I_{2}^{\prime \prime}(h, n) .
\end{aligned}
$$

Since $x^{\alpha} h(x) \rightarrow 0$ as $x \rightarrow \infty$ and $h$ is bounded, it follows that $h(x) \leq K /|x|^{\alpha}+1$ and hence $n h(n x) \leq n k /\left(|n x|^{\alpha}+1\right)$. Therefore

$$
\begin{aligned}
\left\|I_{2}^{\prime \prime}(n, h)\right\| & \leq \int_{A}^{\infty}\left\|\phi_{x_{0}}(f, t)\right\| n|h(n t)| d t \\
& \leq \int_{A}^{\infty}\left\|\phi_{x_{0}}(f, t)\right\| \frac{n k}{n^{\alpha} t^{\alpha}+1} d t \leq \frac{K}{n^{\alpha-1}} \int_{A}^{\infty}\left\|\phi_{x_{0}}(f, t)\right\| \frac{d t}{t^{\alpha}} .
\end{aligned}
$$

Finally let $\Phi(x)=\int_{0}^{x}\left\|\phi_{x_{0}}(f, t)\right\|_{X} d t$. Then

$$
\begin{aligned}
\left\|I_{2}^{\prime}\right\| & \leq \int_{1 / n}^{A}\left\|\phi_{x_{0}}(f, t)\right\|_{X} n h(n t) d t \leq \frac{K}{n^{\alpha-1}} \int_{1 / n}^{A}\left\|\phi_{x_{0}}(f, t)\right\| \frac{d t}{t^{\alpha}} \\
& =\left.\frac{K}{n^{\alpha-1}} \Phi(x) \frac{1}{x^{\alpha}}\right|_{1 / n} ^{A}-\frac{k}{h^{\alpha-1}} \int_{1 / n}^{A} \frac{\Phi(t)}{t} \frac{d t}{t^{\alpha}} .
\end{aligned}
$$

Since $\Phi(x) / x=x^{-1} \int_{0}^{x}\left\|\phi_{x_{0}}(f, t)\right\| d t \rightarrow 0$ as $x \rightarrow \infty$, it follows that $\left\|I_{2}^{\prime}\right\| \rightarrow 0$ as $A \rightarrow 0$ and $n$ stays greater than $1 / A$. Hence in (3.5) let $A$ be so small that (3.6) is less than $\varepsilon$; then for $n$ sufficiently large, (3.5) and (3.6) will both be small. To see that the mapping $\Sigma_{X}\left(h, x_{0}\right)$ is uniformly bounded in the norms of $L_{x_{0}}^{1}$, observe that (3.5) and (3.6) are clearly bounded by $\|f\|_{1}+\left\|\rho\left(x_{0}, f\right)\right\|_{c_{0}}$.

To show commutativity of diagrams, it is only necessary to observe that $\Sigma_{X}\left(h, x_{0}\right)(f)=h_{n} * f$ for every $n$, and by Proposition 1.8 convolution is a natural mapping. Thus the diagram:

$$
\begin{aligned}
& L_{x_{0}}^{1}(X) \stackrel{\Sigma_{X}\left(h, x_{0}\right)}{\longrightarrow} C_{0}(X) \\
& L_{x_{0}}^{1}(\alpha) \downarrow \quad \downarrow C_{0}(\alpha) \\
& L_{x_{0}}^{1}(Y) \underset{\Sigma_{Y}\left(h, x_{0}\right)}{\longrightarrow} C_{0}(Y)
\end{aligned}
$$

As particular cases Theorem 3.1 includes the $(C, \alpha)$ means and the Abel means 
of the Fourier series of a function $f$; the computations by which this may be shown are elementary and well known [45]. As in the case of Theorem 3.0, Theorem 3.1 also has a corollary obtained from the results of $\$ 2$.

Corollary 3.1.1. Let $\mathcal{B}_{\omega}$ be the category of Banach distribution spaces on $T^{1}, L_{0}^{1}$ the functor of Definition 3.4 at $x_{0}=0$ on $\mathcal{B}_{\omega}$, and $\delta_{0}$ the evaluation mapping at 0 on $L_{0}$. Then for every $h \in L^{\infty}$ such that (1) $h$ is even, (2) $\int h d t=1$, (3) $h(x) \sim O\left(x^{\alpha}\right)$ for some $\alpha>1$ and $x$ large, $\Sigma(h, 0)$ converges to $\delta_{0}$ and hence for every $X \in \mathcal{B}_{\omega}$ and $f \in \Lambda \Delta L_{0}^{\prime}(X), h_{n} * f \rightarrow f$ in $X$.

Proof. The proof of Corollary 3.1.1 is the same as that of the previous corollary. If $\tau f \in \Delta L_{0}^{\prime}(X)$, then $h_{n} * f=h_{n} * \tau f(0) \rightarrow \tau f(0)=f$ in $X$.

Example. Let $\mu$ be a Radon-Stieltjes measure on $T^{1}$ and define the space $L_{\mu}^{1}$ of all $\mu$-measurable functions on $T^{1}$ by $L_{\mu}^{1}=\left\{f: T^{1} \rightarrow \mathrm{C} \mid\|f\|_{L}<\alpha\right\}$. $L_{0}^{1}\left(L_{\mu}^{1}\right)$ is therefore equal to the class of all $f \in L_{\mu}^{1}$ such that

$$
\|f\|_{L^{\prime}(L)}=\int_{T^{1}}\left(\int_{T^{1}}\left|f_{s}\right|^{1} d \mu\right) d s<\infty
$$

and $\rho_{0}(f)(n)=r \int_{-1 / n}^{1 / n}\left[\int_{T^{1}}\left|f_{s}\right|^{1} d \mu\right] d s \rightarrow 0$ as $n \rightarrow \infty$. By Corollary 3.1.1 it follows that for every $f \in L_{0}^{1}\left(L_{\mu}^{1}\right), \sigma_{n}^{\alpha} f \rightarrow f$ in the norm of $L_{\mu}^{1}$, where $\sigma_{n}^{\alpha} f$ is the sequence of $(C, \alpha)$ Cesàro means of the Fourier series for the function $f$. In particular, $\mu$ may for instance be a sum of point measures; Corollary 3.1.1 will then reduce to standard conditions for pointwise convergence.

Corollary 3.1.1 applies to any Banach space $X$ of distributions on $T^{1}$ regardless of whether the norm of $X$ is translation invariant. Although there do not seem to be any results on convergence of approximate identities of the type of (3.2) in arbitrary Banach function spaces, one problem prominent in the theory of approximation is similar (though not identical) to that dealt with by Theorem 3.1 and its corollary: the theory of trigonometric interpolation and Jackson polynomials. The Fourier-Lagrange series (see Zygmund [45]) of a function $f$ will define a best approximation to $f$ in a weighted $L^{2}$ space; Jackson polynomials are the $(C, 1)$ means of the Fourier-Lagrange series. Corollary 3.1.1 may be reformulated as stating conditions under which the Cesàro means of the Fourier series itself will converge in a weighted space. In any discussion with respect to the functor $\Delta L_{0}^{1}$ it should be recalled that $\Lambda \underline{\Delta} L_{0}^{1}(X)$ will not necessarily be a closed subspace of $X$.

In his book [15], Katznelson gives a proof that the Cesàro means of a Fourier series on $T^{1}$ converge in norm to $f \in X$ for all spaces $X$ of a class he terms "homogeneous". Homogeneous spaces are Banach spaces contained in $L^{1}$ and having norms invariant and continuous under translation; they are essentially the same as the class of spaces $X \in \mathcal{B}_{0}$ such that $X \subseteq L^{1}$ and $\Lambda C(X)=X$, where $C$ is the continuity functor. Corollary 3.1.1 therefore implies Katznelson's result.

Finally it is clear that other theorems on convergence of convolution kernels or of Fourier series may be generalized in a similar way to the context of 
functors. Both Dini's theorem and Jordan's convergence test may both be generalized to theorems for vector-valued functions; these theorems will then have corollaries similar to Corollaries 3.0.1 and 3.1.1.

2. Inverse and convergence theorems for Lipschitz functors $C^{\prime}$. In the previous section I have proved that for every $h \in L^{1}$ such that $\int h d t, \Sigma(h)-I$ defines a natural mapping from $C$ to $c_{0} C$, and therefore that the sequence $\left\langle\Sigma(h)_{n}(f)\right\rangle$ converges to $f$ in $C(X)$ for every $f \in X$ and every $X \in \mathcal{B}$. These results then imply results for the functor $\Delta C$, valid for any $h \in L^{1}$ with integral $\int h d t=1$.

For stronger functors, the Lipschitz functors $C^{r}$ for $0<r<1$, it is possible to prove very much stronger theorems than these. Using a striking theorem recently proved by $H$. Shapiro [38], I shall prove here an inverse result on the convergence of $f * h_{n}=\Sigma(h)_{n}(f)$. Inverse results of this type are well known in approximation theory; they state exact conditions both necessary and sufficient for the convergence of $\Sigma(h)(f)$ to $f$ at a given rate. Finally, using a well known result of Kolmogorov combined with Corollary 4.5.1 from Chapter 4, I shall show the convergence of the Fourier series of a function for the functor $C_{0}^{\prime}$. These results will then lead by Theorem 2.4 to corresponding results for the functor $\Lambda C^{\prime}$ and thus for all spaces $\Lambda C^{r}(X)$ with $X \in \mathcal{O} M_{0}^{\prime}$.

I shall first define the functors and mappings of functors by which these results may be stated.

Definition 3.5. Let $\mathcal{B}$ be the category of Banach spaces and $0<r<1$. Define a functor

$$
\begin{aligned}
c^{r}(X) & =\left\{f: Z^{+} \rightarrow X \mid\left\|n^{r} f(n)\right\|_{X} \leq K\right\}, \\
\|f\|_{c^{\prime}(X)} & =\inf \left\{K \mid\left\|n^{\prime} f(n)\right\|_{X} \leq K \forall n\right\}, \\
c^{r}(\alpha) & =\alpha f(n),
\end{aligned}
$$

for every $X \in \mathcal{B} . Z^{+}$denotes the nonnegative integers.

By Theorem 3.0 above, for every $h \in L^{1}$ such that $\int h d t=1, \Sigma(h)$ converges to the identity mapping on the functor $C$. Combining Definition 3.2 with Definition 3.5 it is therefore possible to define

Definition 3.6. Let $\mathcal{B}$ be the category of Banach spaces and $C$ the continuity functor on $\mathcal{B}$ over $T^{1}$. Let $h \in L^{1}$ and define, for every $X \in \mathcal{B}$,

$$
\begin{aligned}
\Sigma^{\prime} C(h, X) & =\left\{f \in C(X) \mid \Sigma(h)(f)-f \in c^{\prime} C(X)\right\}, \\
\|f\|_{\Sigma^{\prime} C(h, X)} & =\|\Sigma(h, f)\|_{c^{\prime} C(X)}+\|f\|_{C(X)}, \\
\Sigma^{\prime} C(h, \alpha) & =\alpha f(x) .
\end{aligned}
$$

The functor $\Sigma^{r} C(h)$ essentially describes for every $X \in \mathcal{B}$ the class of $f \in C(X)$ such that $\Sigma(h)_{n}(f)=f * h_{n}$ goes to $f$ at a rate given by the constant $r$ in $c^{\prime}$. 
It is now possible to state and prove the inverse theorem.

Theorem 3.2. Let $T^{1}$ be the one-dimensional toroidal group, let $0<r<1$, and let $\mathcal{B}$ be the category of Banach spaces. Finally let $C^{r}$ be the Lipschitz functor (Definition 1.10) on $T^{1}$. Then for every $h \in L^{1}$ such that $\int h d t=1$, there exists a natural isomorphism

$$
\Sigma^{r} C(h) \stackrel{i h}{=} C^{r}
$$

Proof. This theorem follows very easily by the same methods used in the proof of Corollary 3.1 in H. Shapiro [38]. However it is necessary to explicitly obtain constants left implicit in Shapiro, in order to prove the naturalness of the isomorphism $i_{h}$.

Let $\sigma$ be a measure on $R^{1}$. For every $s \geq 0$, define $\sigma^{s}$ by $\int f(s u) d \sigma_{u}=\int f(u) d \sigma_{u}^{s}$ for every $f \in C$. Thus for instance if $d \sigma=h(u) d u$ where $h$ is integrable, $d \sigma^{s}=s^{-1} h(u / s) d u$. Furthermore for every $f \in C$, let $D_{o}(f, s)=\left\|f * \sigma^{s}\right\|_{\infty}$.

A measure $\sigma$ on $R^{1}$ is said to satisfy the Tauberian condition if there exist points $x_{1}, x_{2}$ such that $x_{1}>0>x_{2}$ and $\hat{\sigma}\left(x_{1}\right) \neq 0, \hat{\sigma}\left(x_{2}\right) \neq 0$.

Finally let $\exists M$ denote the image of $M$ under the Fourier transform on $R$.

Lemma 3.2.1. Let $C$ be the space of continuous complex-valued functions on $\boldsymbol{R}^{\mathbf{1}}$, let $\sigma$ and $\rho$ be measures on $R^{1}$, and suppose that $\sigma$ satisfies the Tauberian condition and $\hat{\rho}$ vanishes in a neighbourhood of 0 . Then for every $f \in C$ and $s>0$, there exist constants $A, B_{j}$, and $b, 0<b<1$, independent of $f$, such that

$$
D_{\rho}(f, s) \leq A \sum_{k=0}^{\infty} \sum_{j=1}^{\infty} D_{o}\left(f, B_{j} b^{k} s\right) .
$$

This lemma is Corollary 1.1 to Theorem 1 of $\mathrm{H}$. Shapiro [38]. The basic idea of the proof stems from the observation that if $\rho$ and $\sigma$ are measures and there exists a measure $\tau$ such that $\rho=\sigma * \tau$, then $D_{\rho}(f, s) \leq\left\|\tau_{1}\right\| D_{o}(f, s)$. The general case of the theorem (in which the equation $\hat{\rho}=\hat{\sigma} \cdot \hat{\tau}$ holds only near 0 ) is reduced to this case by multiplying $\hat{\rho}$ and $\hat{\sigma}$ by a suitable function $P$ with compact support.

Lemma 3.2.2. Let $\sigma, \rho$ be measures on $R^{1}$. Suppose that

(1) $\sigma$ satisfies the Tauberian condition;

(2) there exists a function $P$ and a neighbourhood $N$ of 0 such that:

(a) $P(b x)=b^{q} P(x)$, for $x \in N$,

(b) $P$ and $\hat{\rho} / p$ are both equal to functions in $\exists M$ on $N$. Then if $0<r<q$ and $D_{o}(f, s) \leq K s^{\prime}$, then $D_{\rho}(f, s) \leq A K_{s}$ where $A$ does not depend on the function $f$.

Proof. First, one may suppose that $\hat{\rho}$ is positive homogeneous in a neighbourhood $N$ of 0 . Let $\pi$ be a measure satisfying the Tauberian condition such that $\hat{\pi}(x)=P(x)$ for $x \in N$. Then if Lemma 3.2.2 holds for $D_{z}(f, s)$, it follows for $\rho$ by Lemma 3.2.1. I shall therefore suppose $\rho$ is homogeneous. 
Let $T(x)=\hat{\rho}(x)-2^{-r} \hat{\rho}(2 x)$ and $\hat{\tau}(x)=T(x)$. By assumption $\hat{\rho}$ is homogeneous; hence $T(x)=0$ in a neighbourhood of 0 . Hence by Lemma 3.2.1

$$
D_{r}(f, s) \leq A_{0} s^{r}
$$

where $A_{0}$ does not depend on $f$. Since

$$
\hat{\rho}(x)=\sum_{n=0}^{\infty} 2^{n(1-q)}\left(\tau^{2-n}\right)^{\wedge}=\sum_{n=0}^{\infty} 2^{-n q} T\left(2^{n} x\right),
$$

it follows by (3.8) that

$$
D_{p}(f, s) \leq \sum_{n=0}^{\infty} 2^{-n q} D_{r}\left(f, 2^{n} s\right) \leq A_{1} \sum_{n=0}^{\infty} 2^{-n q} \min \left(\left(2^{n} s\right)^{r}, 1\right) .
$$

Since $r<q, D_{p}(f, s) \leq A s^{r}$ where $A$ does not depend on either $f$ or $s$.

I shall first prove the theorem for the case of scalar-valued functions; it may then be extended by reducing the vector case to the scalar case through linear functionals. Let $\omega_{2}(f, s)=D_{\beta_{2}}(f, s)=\left\|f_{2 s}-2 f_{s}+f\right\|_{6}$ where $\beta_{2}$ is the measure with masses $1,-2,1$ at $0,1,2$ respectively. $\hat{\beta}_{2}(x)=\left(1-e^{-i x}\right)^{2}$ and the function $P(x)=(x)^{2}$ satisfies the conditions of Lemma 3.2.2.

Let $\delta$ be the measure with unit mass at 0 and suppose that $h \in L^{1}$. do $=d \delta-h d x$ will then define a measure which satisfies the Tauberian condition $\hat{\sigma}=1-\hat{h}$ and $\hat{h}(x) \rightarrow 0$ as $x \rightarrow \infty$. Therefore the conditions of Lemma 3.2.2 are satisfied and

$$
\left\|f_{2 s}-2 f_{s}+f\right\|_{b_{0}} \leq A K s^{\prime}
$$

where $A$ is independent of $f$. For $0<r<1$ it is well known (see, for instance, Zygmund [43] or Taibleson [41]) that (3.9) implies that $\left\|f_{s}-f\right\|_{\infty} \leq A K s^{\prime}$.

To obtain the theorem for the scalar case it is now only necessary to consider the closed subspace of $C$ consisting of all functions with period $2 \pi$.

To extend the theorem to the vector-valued case, let $X \in \mathcal{B}$ and $f \in C(X)$, and suppose that $f \in \Sigma^{\prime} C(h, X)$. Then

$$
\left\|f * h_{n}-f\right\|_{C(X)}=\left\|\Sigma(h)_{n} f-f\right\|_{C(X)} \leq\|f\|_{\Sigma^{\prime} C(h, X)^{n^{-r}}} .
$$

Then for every $x^{*} \in X^{*}$ such that $\left\|x^{*}\right\| \leq 1$, and every $s \in T^{1}$,

$$
\begin{aligned}
\left\|\left\langle x^{*}, f-h_{n} * f\right\rangle\right\| & =\sup _{s}\left|\left\langle x^{*}, f(s)-h_{n} * f(s)\right\rangle\right| \\
& \leq \mid f-h_{n} * f \|_{C(x)} \leq K n^{-r}
\end{aligned}
$$

where $K=\|f\|_{\Sigma^{\prime} C(h, X)}$ and thus does not depend on $x^{*}$. Hence

$$
\left|\left\langle x^{*}, f_{1 / n}(s)-f(s)\right\rangle\right| \leq\left\|\left\langle x^{*}, f_{1 / n}-f\right\rangle\right\|_{\infty} \leq A K n^{\prime}
$$


where $A$ is the constant defined by (3.9), and also does not depend on $x^{*}$. Thus since (3.10) holds for all $x^{*} \in X^{*},\left\|f_{1 / n}-f\right\|_{C(X)} \leq A K n^{-r}$ and hence $\left\|f_{s}-f\right\|_{C(X)}$ $\leq A K s^{-r}$ which implies that $f \in C^{\prime}(X)$ and $\|f\|_{C^{\prime}(X)} \leq A\|f\|_{\Sigma^{\prime} C(h, X)}$.

It is clear that if $f \in C^{r}(X)$, then $f \in \Sigma^{r} C(h, X)$. To establish the isomorphism $i_{h}$ of (3.7), let $i_{h, X}(f)=f$ for every $X \in \mathcal{B}$ and $f \in \Sigma^{r} C(h, X)$. Since $i_{h, X}$ is the identity mapping on $\Sigma^{r} C(h, X), i_{h}$ clearly defines a natural mapping of functors. The theorem is proved.

By using the properties of the mapping $\tau$ and $\Lambda$, it is possible to obtain the following corollary.

Corollary 3.2.1. Let $T^{1}$ be the one-dimensional toroidal group, let $0<r<1$ and let $M_{0}^{\prime}$ be the category of normal modules over $M_{0}$ in $\mathcal{B}_{0}$. Finally let $C^{\prime}$ be the Lipschitz function acting on $M_{0}$ over $T^{1}$. Then for every $h \in L^{1}$ such that $\int h d t=1$, there exists a natural isomorphism $i_{h}$,

$$
\Lambda \Sigma^{r} C(h) \stackrel{i_{h}}{\cong} \Lambda C^{\prime} \text {. }
$$

Therefore in particular for any $X \in \mathcal{} M_{0}, h_{n} * f \rightarrow f$ to the order $O\left(n^{-r}\right)$ if and only if $f \in \Lambda C^{r}(h, X)$.

Proof. Let $f \in \Lambda \Sigma^{r} C(h, X)=\left\{f \in X \mid\left\|h_{n} * f-f\right\|_{X} \leq O\left(n^{-r}\right)\right\}$. If $f \in$ $\Lambda \Sigma^{\prime} C(h, X), \tau f \in \Sigma^{\prime} C(h, X)$ and thus, for some $K>0$,

$$
\left\|\tau f-\tau\left(h_{n} * f\right)\right\|_{C(X)}=\left\|\tau f-h_{n} * \tau f\right\|_{C(X)} \leq k n^{-r} .
$$

Therefore by Theorem 3.2, $\left\|(\tau f)_{s}-\tau f\right\|_{C(X)} \leq A K s^{-r}$.

Since, by Remark 2.0, $\tau$ commutes with translation,

$$
\left\|f_{s}-f\right\|_{X}=\left\|\tau\left(f_{s}-f\right)\right\|_{C(X)}=\left\|(\tau f)_{s}-\tau f\right\|_{C(X)} \leq A K s^{-r}
$$

and the corollary is proved.

Remark. It should be observed here that in his paper [38], Shapiro suggests the possibility of a treatment of inverse theorems for a much more general class of norms than the norms $C, C^{r}$ he treated in [38]. He also proves an inverse theorem for the $L^{p}$ norm.

It is clear that the case of either the Cesàro $(C, \alpha)$ means of the Fourier series of $f$, or the Abel means, is contained as a special case of Theorem 3.2. For the Fourier series itself of a function $f$ it is possible to prove a quite strong result for a subfunctor of the functor $C^{r}$.

Let $D$ be the function defined by $D(x)=(\sin x) / x$, and let $D_{n}(x)$ $=(\sin n x) / n x$. The function $D$ is not integrable; however for every $\varphi \in L^{1}$ and extended by periodicity to $R^{1}$ :

$$
\int \varphi D_{n} d t=\lim _{N \rightarrow \infty} \int_{-N}^{N} \varphi D_{n} d t
$$


exists (see Zygmund [45, II.7]). It is well known that in the wk* sense the integral (3.11) defines the Dirichlet kernel of the Fourier series, and that $f * D_{n}(x)$ $=\sum_{-n}^{n} f(v) e^{i v x}$ is the Fourier series of $f$.

I shall therefore state

Definition 3.7. Let $B$ be the category of Banach spaces and $L^{1}$ the $L^{1}$ functor on $\mathcal{B}$ over $T^{1}$. Define, for every $X \in \mathcal{B}, f \in L^{1}(X)$, and integer $n$,

$$
\Sigma(D)_{n}(f)=f * D_{n}=\int f(x-t) n D(n t) d t
$$

where $D_{n}$ is the wk limit defined by the integral (3.11).

The subfunctor $C_{0}^{\prime}$ of $C^{\prime}$ must also be defined.

Definition 3.8. Let $\mathcal{B}$ be the category of Banach spaces, $0<r<1$, and let $C^{\prime}$ be the Lipschitz functor on $\mathcal{B}$ over $T^{\mathfrak{l}}$. Define a functor

$$
C_{0}^{\prime}(X)=\operatorname{cl}_{C^{\prime}(X)}\left\{f \in C^{\infty}(X)\right\} .
$$

$C_{0}^{\prime}(X)$ is the closure in the norm of $C^{\prime}(X)$ of the infinitely differentiable $X$. valued functions

$$
\|f\|_{C^{\prime}(X)}=\|f\|_{C^{\prime}(X)}, \quad C_{0}^{r}(\alpha) f=C^{r}(\alpha) f .
$$

It is clear that $C_{0}^{r}$ defines a closed subfunctor of the functor $C^{r}$.

The following proposition may illuminate some of the significance of the functor $C_{0}^{r}$.

Proposition 3.1. Let $\mathcal{B}$ be the category of Banach spaces and $C^{\prime}$ the Lipschitz functor on $\mathcal{B}$ over $T^{1}$. For every $X \in \mathcal{B}$ let $T_{s} f=f_{s}$ be the operator of translation of $f \in C^{\prime}(X)$ by $s \in T^{\prime}$. Then

(1) $\left\|f_{s}-f\right\|_{C^{\prime}(X)} \rightarrow 0$ as $s \rightarrow 0$ if and only if $f \in C_{0}^{\prime}(X)$.

(2) $C_{0}^{\prime}(X)=\Lambda C \circ C^{\prime}(X)$ for every $X \in \mathcal{B}$.

Proof. The second statement is merely a restatement of the first. It will therefore be sufficient to show the first.

Let $\left\|f_{s}-f\right\|_{C^{\prime}(X)} \rightarrow 0$ as $s \rightarrow 0$ and $f \in C^{\prime}(X)$. Then if $\left\langle\varphi_{k}\right\rangle$ is a $C^{\infty}$ approximate identity such that supp $\varphi_{k} \subseteq(-1 / k, 1 / k)$,

$$
\left\|\varphi_{k} * f-f\right\|_{C^{\prime}(X)} \leq \int_{-1 / k}^{1 / k}\left|\varphi_{k}(t)\right|\left\|f_{t}-f\right\|_{C^{\prime}(X)} d t \rightarrow 0 \text { as } k \rightarrow \infty
$$

and hence $f \in C_{0}^{\prime}(X)$. It is clear that since $\left\|\varphi_{s}-\varphi\right\|_{C^{\prime}(X)} \rightarrow 0$ as $s \rightarrow 0$ for every $\varphi \in C^{\infty}(X)$, the same must hold for every $f \in C_{0}^{r}(X)$.

The following theorem for the functors $C_{0}^{r}$ may now be shown.

Theorem 3.3. Let $B$ be the category of Banach spaces, $0<r<1$, and let $C_{0}^{\prime}$ be the Lipschitz functor (3.11) of Definition 3.8. Suppose that $I$ is the identity mapping on the functor $C_{0}^{r}$. Then $\Sigma(D)$ converges to I on $C_{0}^{\prime}$. 
Proof. This proof will follow an observation made by Kolmogorov for $\boldsymbol{L}^{\boldsymbol{P}}$. Let $X \in \mathcal{B}$ and $f \in C_{0}^{r}(X)$. It will be sufficient to show that the inequality

$$
\left\|\Sigma(D)_{n} f\right\|_{C^{\prime}(X)}=\left\|D_{n} * f\right\|_{C^{\prime}(X)} \leq K\|f\|_{C^{\prime}(X)}
$$

holds for every $X \in \mathcal{B}$ and $f \in C^{\prime}(X)$ with a constant $K$ independent of $X$. The theorem will then follow from the observation that

$$
\left\|D_{n} * f-f\right\|_{C^{\prime}(X)}=\left\|\Sigma(D)_{n} f-f\right\|_{C^{\prime}(X)} \rightarrow 0 \text { as } n \rightarrow \infty
$$

for every $f \in C^{\infty}(X)$.

Let $H$ denote the Hilbert transform

$$
H f(s)=\lim _{e \rightarrow 0} \int_{e \leq|1 / 4| \leq \pi} f(t) \cos \frac{1}{2}(s-t) d t .
$$

It is well known that $H$ defines a distribution with Fourier transform $\boldsymbol{H}(n)$ $=\operatorname{sgn} n \forall n \in Z$, and furthermore $H: C^{r} \rightarrow C^{r}$ where $C^{r}$ is the Lipschitz space. Therefore by Corollary 4.4.1 below it follows that $H$ defines a natural mapping of the Lipschitz functor $H: C^{r} \rightarrow C^{r}$.

Let $X \in \mathcal{B}$ and $f \in C^{r}(X)$. Define $f_{ \pm N}(s)=e^{ \pm i N x} f(s)$. Then

$$
\begin{gathered}
H * f_{N}(s)=-i \Sigma \operatorname{sgn} n \hat{f}(n-N) e^{i n s}, \\
H * f_{-N}(s)=-i \Sigma \operatorname{sgn} n \hat{f}(n+N) e^{i n s},
\end{gathered}
$$

and therefore

$$
\begin{aligned}
\Sigma(D)_{n} f(s) & =D_{n} * f(s) \\
& =(i / 2)\left[e^{-i N s}\left[H * f_{N}(s)\right]-e^{i N s}\left[H * f_{-N}(s)\right]\right]-\hat{f}(N) e^{i N x}-\hat{f}(-N) e^{-i N x} .
\end{aligned}
$$

Inequality (3.13) therefore follows at once from the representation (3.14) and the fact that $H$ is a natural mapping from $C^{r}$ to $C^{r}$. The theorem is proved.

Theorem 3.3 has a corollary which follows immediately from Theorem 2.4.

Corollary 3.3.1. Let $M_{0}^{\prime}$ be the category of normal Banach modules in $\mathcal{B}_{0}$, over $M_{0}$, let $0<r<1$, and let $C_{0}^{r}$ be the Lipschitz functor on $M_{0}$. Let $I$ be the identity mapping on $C_{0}^{r}$. Then $\Sigma(D)$ converges to $I$ on $\Lambda C_{0}^{r}$. Therefore in particular for every $X \in M_{0}^{\prime}, D_{n} * f \rightarrow f$ in $\Lambda C_{0}^{r}(X)$ for every $f \in \Lambda C_{0}^{r}(X)$.

Proof. Let $f \in \Lambda C_{0}^{r}(X)$ and $X \in M_{0}$. Then $\tau f \in C_{0}^{r}(X)$. By Theorem 2.4, $D_{n} * \tau f$ is in $C_{0}^{\prime}(X)$. By Remark 2.0.1, $\tau$ commutes with convolution. Hence $D_{n} * \tau f=\tau\left(D_{n} * f\right)$ and therefore

$$
\left\|D_{n} * f-f\right\|_{\Lambda C_{0}^{\prime}(X)}=\left\|\tau\left(D_{n} * f-f\right)\right\|_{C_{b}(X)}=\left\|D_{n} * \tau f-\tau f\right\|_{C_{b}(X)} \rightarrow 0
$$

as $n \rightarrow \infty$, and the corollary is proved. 
In [15], Katznelson proves a theorem which I shall state here for comparison with Corollary 3.3.1.

Theorem 3.4 (Katznelson). Let $X$ be a homogeneous Banach space and $H$ the Hilbert transform. Then $H: X \rightarrow X$ if and only if the Fourier series $D_{n} * f$ of $f$ converges to $f$ in the $X$-norm for every $f \in X$.

Theorem 3.4 may be reformulated to the following.

Theorem 3.4A. Let $M_{H}$ be the algebra formed by adjoining the Hilbert transform $H$ to $M_{0}$. Let $X \in M_{0}^{\prime}$ and $\Lambda C(X)=X$. Then $X$ is a module over $M_{H}$ if and only if $D_{n} * f \rightarrow f$ in $X$ for every $f \in X$.

Corollary 4.4.1 would seem to add particular interest to Katznelson's result in that it provides a means of obtaining a very large number of spaces $X$ with the properties he describes.

\section{CHAPTER 4: TENSOR CONVOLUTION MULTIPLIERS}

In this chapter I shall give a complete characterization of the tensor multipliers $\mathrm{Hom}_{\circledast}[F, F]$ for the tensor multipliers generated by the leading continuity and boundedness functors $L^{\infty}, C$, and $C^{r}$ for $0<r<1$. These results will then yield strong inclusion relations for the multipliers on very large classes of Banach modules. These relationships seem most striking in the case of CalderónZygmund operators, which will be seen to be multipliers on all Banach modules of the form $\Lambda C^{r}(X)$ for $X$ a normal Banach module contained in $M_{0}^{\prime}$.

As mentioned after Definition 1.13, the functor $\operatorname{Hom}_{\otimes}[F, G]$ is the analogue for the functors $F$ and $G$ of the Banach spaces $[X, Y]$ of convolution multipliers from the Banach modules $X$ to $Y$. Algebras of convolution multipliers have been extensively studied by many authors in the context of harmonic analysis.

As in the case of the treatment of pointwise convergence given in Chapter 3, I shall not discuss the question of multipliers for the Hilbert functor $L^{2}$. I will also omit a discussion of the $L^{p}$ functors for $p \neq 2$. Although $L^{p}$ spaces for $1<p<\infty$ have so far played a very prominent role in the theory of convolution multipliers, most results relating to them seem to depend on quite special properties of $L^{p}$ spaces of complex-valued functions themselves. On the other hand the notion of tensor multipliers allows one to deal quite freely with multipliers $H$ which are themselves vector-valued, acting on vector-valued spaces. In this way the idea of tensor multipliers allows one to give a unified treatment of such cases as that of integral operators dependent on a parameter or themselves having operator values. Several authors [1], [14], [34], have studied vector-valued $\left(L^{p}, L^{p}\right)$ multipliers with the intention of developing a unified treatment of this kind; a quite compact theory is possible for the functor $C^{r}$.

1. General properties of convolution multipliers on Banach modules. In the case in which the Banach modules consist of distributions taking only complex values, 
it is possible to obtain some strong and useful theorems on the properties and character of the convolution multipliers. In this section I shall develop the basic properties of convolution multipliers as operators on Banach modules of complex-valued distributions on a toroidal group $T^{n}$.

Let $\mathcal{B}$ be the category of Banach spaces, $T^{n}$ a toroidal group, and $D_{n}$ the Schwartz distribution functor over $T^{n}$ acting on the category $\mathcal{B}$. Let $\mathcal{M}_{00}$ be the category of modules by convolution over the ring $M_{0}$, consisting of complexvalued distributions, and define $\boldsymbol{\alpha} M_{0}$ to be the corresponding category of modules of Banach space-valued distributions. Finally for every $Z \in \mathcal{B}$, define $\mathcal{M}_{0}(Z)$ to be the category of modules (over $M_{0}$ ) of $Z$-valued distributions. The corresponding class of mappings for $\mathcal{M}_{0}(Z)$ consists of all module homomorphisms $[X, Y]$ for $X, Y \in \propto M_{0}(Z)$.

Definition 4.0. Let $Z \in \mathcal{B}$ and $H \in D_{n}(Z)$. I shall say that $H$ is a convolution multiplier from $X \in M_{0}$ to $Y \in \mathcal{O} M_{0}(Z)$ if $H$ defines a continuous module homomorphism $H \in[X, Y]$ by $H f=H * f$ for every $f \in X$.

Remark. It is an immediate consequence of Schwartz [36, II, Proposition 35] that the convolution of a complex-valued distribution $f$ and a Banach spacevalued distribution $H$ is uniquely defined.

The following proposition now follows easily.

Proposition 4.0. Let $M_{0}$ be the category of modules on $T^{n}$ over $M$, and suppose

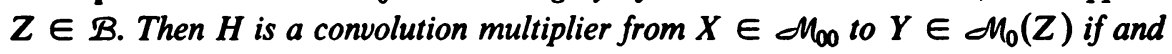
only if $H \in[X, Y]$.

Proof. The "only if" part of this proposition is clear since it is identical to Definition 4.0. It therefore remains only to show the second part. If $H \in[X, Y]$, then $H$ commutes with $M$ and therefore commutes with all trigonometric polynomials $p$.

Let $Z_{X}=\left\{m \in Z^{n} \mid \exists f \exists X \in f(m) \neq 0\right\}$. Then $Z_{X} \neq \varnothing$, for if not, then for every $f \in X, f * e_{m}=0$ where $e_{m}(x)=e^{i\langle m, x\rangle}$ and therefore $\left\langle f, e^{-m}\right\rangle=0$ for every $m \in Z^{n}$. Since the set of all trigonometric polynomials $p$ is dense in $C^{\infty}$, this implies $f=0$ for every $f \in X$.

Furthermore $e_{m} \in X$ for every $m \in Z_{X}$; since $X$ is a module over $M$, $f * e_{m}=\hat{f}(m) e_{m} \in X$, and if $m \in Z_{X}$, then $e_{m} \in X$.

Since $X$ is a module over $M_{0}$ and $e_{m} \in X$ for every $m \in Z_{X}, H\left(e_{m} * e_{m}\right)$ $=e_{m} * H\left(e_{m}\right)=H\left(e_{m}\right)^{\wedge}(m) e_{m}$, where $H\left(e_{m}\right)^{\wedge}(m)$ takes values in the Banach space $Z$ for every $m \in Z^{n}$. Furthermore if $m \notin Z_{X}$, then for every $f \in X$, $e_{m} * H f=H\left(e_{m} * f\right)=H(0)=0$. Hence $\hat{e}_{m} \cdot(H f)^{\wedge}(m)=0$ and therefore $(H f)^{\wedge}(m)=0$ for every $m \notin Z_{X}$ and $f \in X$.

Let

$$
\begin{aligned}
\phi(m) & =\left(H e_{m}\right)^{\wedge}(m) & & \text { for every } m \in Z_{X}, \\
& =0 & & \text { otherwise. }
\end{aligned}
$$


Since $H$ is linear, $(H p)^{\wedge}(m)=\phi(m) \hat{p}(m)$ for every $m \in Z^{n}$ and every trigonometric polynomial $p$. Furthermore for every $f \in X$ and $m \in Z^{n}$,

$$
\begin{aligned}
& {[H(p * f)]^{\wedge}(m)=\hat{p}(m) \cdot(H f)^{\wedge}(m),} \\
& {[H(p * f)]^{\wedge}(m)=\phi(m)(p * f)^{\wedge}(m)=\phi(m) \hat{p}(m) \hat{f}(m),}
\end{aligned}
$$

where the second relation follows from the fact that $p * f$ is a trigonometric polynomial if $p$ is. Hence from (4.0) it follows that for every $m \in Z^{n},(H f)^{n}(m)$ $=\phi(m) \hat{f}(m)$.

To show the proposition it only remains to show that $\phi$ is a tempered sequence, from which it will follow that $\phi$ is the Fourier transform of a distribution in $D(Z)$. To do so, one observes that since $H: X \rightarrow Y$ continuously, $H: X \rightarrow D(Z)$.

Suppose that $\phi$ is not a tempered sequence. Then there exists a sequence $\left\langle m_{k}\right\rangle \in Z^{n}$ such that $\left|m_{k}\right| \rightarrow \infty$ and $\phi\left(m_{k}\right)>\left|m_{k}\right|^{k}$. Clearly every $m_{k}$ must be in $Z_{X}$ by (4.0); hence it follows that the function

$$
g(x)=\sum_{0 \leq\left|m_{k}\right|<\infty}\left|m_{k}\right|^{-k / 2} e^{i\left\langle m_{k}, x\right\rangle}
$$

is in $X$ since each $e m_{k} \in X$; therefore

$$
\left\|(H g)^{n}\left(m_{k}\right)\right\|_{X}=\left\|\phi\left(m_{k}\right) \hat{g}\left(m_{k}\right)\right\|_{X}>\left|m_{k}\right|^{k / 2} \text {. }
$$

This contradicts the fact that $H g \in D(Z)$ and therefore is tempered.

Remarks. I do not know the origins of this characterization of convolution multipliers. A very similar theorem, with the class $C^{\infty}$ taking the place of $M_{0}$, was proved by Laurent Schwartz [35]. In its complex-valued form Proposition 4.0 seems to be common knowledge, in that several authors have mentioned its possibility or referred to it in some way: Rieffel [31], Edwards [11].

The following theorem provides a useful characterization of the class of normal Banach modules.

Theorem 4.0. Let $X$ be a Banach space of complex-valued distributions on $T^{n}$ and let $M_{0}=L^{1} \oplus H_{0}$. Then $X$ is a normal Banach module over $M_{0}$ if and only if $X$ is a harmonically invariant Banach space. Therefore as classes of Banach spaces $M_{00}$ and $\mathcal{B}_{00}$ coincide.

Proof. Suppose first that $X$ is a normal Banach module over $M_{0}=L^{1} \oplus H_{0}$. Then clearly the norm $\|\cdot\|_{X}$ of $X$ is translation invariant, since, for every $\lambda \in X$, $T_{s} \lambda=\lambda_{s}=\delta_{s} * \lambda$, where $\delta_{s}$ is the point measure at $s$, and $\left\|\lambda_{s}\right\| \leq\|\lambda\|$ for every $s \in T^{n}$. Hence $\left\|\lambda_{s}\right\|=\|\lambda\|$ for every $s$. Since $X$ is a module over $L^{1}$ and $e_{m} \in L^{1}$ for every exponential $e_{m}, \hat{\lambda}(m) e_{m}=\lambda * e_{m} \in X$ for every $m$. Hence $e_{m} \in X$ for every $m$ such that there exists $\lambda \in X \ni \hat{\lambda}(m) \neq 0$; therefore by Definition 1.18, $E_{X} \subseteq X$. Since $X$ is normal, the norm $\|\lambda\|_{X}^{\prime}=\sup _{\|h\|_{1} \leq 1}\|\lambda * h\|_{X} \sim\|\lambda\|_{X}$. The space $X^{*}$ dual to $X$ is also a Banach module over $M_{0}$ by duality. Let 


$$
\begin{aligned}
C_{X}^{\infty} & =\left\{\varphi \in C^{\infty} \mid \varphi * e_{m}=0 \forall e_{m} \notin E_{X}\right\}, \\
C_{X^{1}}^{\infty} & =\left\{\varphi \in C^{\infty} \mid \varphi * e_{m}=0 \forall e_{m} \in E_{X}\right\}, \\
V & =\operatorname{cl}_{X} \cdot \operatorname{span}\left\{h * x^{*} \mid h \in L^{1}, x^{*} \in X^{*}\right\}
\end{aligned}
$$

Since $C^{\infty}$ is dense in $L^{1}$ and $C^{\infty}=C_{X}^{\infty} \oplus C_{X^{1}}^{\infty}$, it follows that $C_{X} * X^{*}$ is dense in $V$. However, for every $e_{m} \in E_{X}$ and for every $x \in X$,

$$
\left\langle e_{m} * x^{*}, x\right\rangle=\left\langle x^{*}, x * e_{m}\right\rangle=\left\langle x^{*},\left\langle x, e_{m}\right\rangle e_{m}\right\rangle=\left\langle\left\langle x^{*}, e_{m}\right\rangle e_{m}, x\right\rangle .
$$

Since Span $E_{X}$ is dense in $C_{X}^{\infty}$, it follows that $C_{X}^{\infty}$ is dense in $V$. Finally $V$ is a norming subspace of $X^{*}$, since $X$ is normal and

$$
\begin{aligned}
\sup _{\|v\|_{x} \leq 1}\langle v, x\rangle & =\sup _{\| h h_{1} \leq 1} \sup _{\left\|x^{*}\right\| \leq 1}\left\langle h * x^{*}, x\right\rangle=\sup _{\|h\|_{b} \leq 1} \sup _{\left\|x^{*}\right\| \leq 1}\left\langle x^{*}, x * h\right\rangle \\
& =\sup _{\|h\|_{1} \leq 1}\|x * h\|_{x} \sim\|x\|_{X} .
\end{aligned}
$$

Suppose now that $X$ is harmonically invariant and thus $X \in \mathcal{B}_{00}$. Then by Theorem 2.3 of Chapter 2, $\Lambda C(X) \subseteq X \subseteq \Lambda L^{\infty}(X)=W(X), X$ is a closed subspace of $W(X)$, and the norm of $W(X)$ is equivalent to the norm of $X$. By Corollary 4.4.1 below, $\|h * x\|_{W(X)} \leq\|h\|_{1}\|x\|_{W(X)}$ and $h * x \in X$ for every $h \in L^{1}$ and $x \in X$. Since the norm of $W(X)$ is invariant with respect to translation, and $X$ is invariant with respect to translation, it follows that under the $W(X)$ norm, $X$ is also a module over $H_{0}$. It is immediately clear from the definition of $W(X)=V\left(X^{*}\right)^{*}$ that

$$
\begin{aligned}
\|x\|_{W(x)} & =\sup _{\|v\|_{x^{*}} \leq 1}\langle v, x\rangle=\sup _{\|h\|_{b} \leq 1} \sup _{\|v\|_{x^{*}}}\langle v * h, x\rangle \\
& =\sup _{\|h\|_{b} \leq 1} \sup _{\|v\|_{x^{*}} \leq 1}\langle v, h * x\rangle=\sup _{\|h\|_{1} \leq 1}\|h * x\|_{W(x)} .
\end{aligned}
$$

Theorem 4.0 states the boundaries of the theory developed in this paper: that essentially the class of normal Banach modules over $M_{0}$ provides the class of all those spaces to which the harmonic operations are appropriate. A similar characterization, though more complex due to the need to include $b e_{m}$ in the Banach module $X$ for every $e_{m} \in E_{X}$, may be given for the class of normal Banach modules of vector-valued distributions.

Examples. It is possible to give several examples and counterexamples to illustrate the classes described by Theorem 4.0.

Example 1. $A$ translation invariant Banach space which is not a module over $M_{0}$. Let $H$ be the set of finite sums $\left\{\lambda=\Sigma^{N} \alpha_{k} \delta_{s_{k}}, s_{k} \neq s_{k}^{\prime} \Rightarrow k \neq k^{\prime}\right\}$ where the $\left\{\delta_{s_{k}}\right\}$ are point measures on $T^{n}$. Endow $H$ with the norm

$$
\|\lambda\|_{B}=\Sigma^{N}\left|\alpha_{k}\right|
$$


Let $A$ be the completion of $H$ under the norm (4.1).

It is clear that $\boldsymbol{H}$ is neither harmonically invariant nor a normal module over $M_{0}$, since $H$ contains no exponentials. However $H$ is still invariant under translation.

It should also be noted here that $\Lambda C(\hat{H})=\varnothing$ while $\Lambda L^{\infty}(\hat{A})=M$, the space of Radon-Stieltjes measures.

Example 2. A Banach module over $L$ which is not invariant under translation. Let $L^{1}$ be the space of integrable functions on $T^{1}$, and $H_{0}^{\prime}$ the linear manifold of point measures with the points $0, \pi$ deleted. Endow $H_{0}^{\prime}$ with the norm

$$
\|\lambda\|_{A_{0}}=\Sigma\left|\alpha_{k}\right| / \sin \left|x_{k}\right| \text { for } \lambda=\Sigma \alpha_{k} \delta_{x_{k}} .
$$

Finally let $X=L^{1} \oplus A_{0}$. Then $X$ is a Banach module over $L^{1}$, but $X$ is clearly not invariant under translation.

Example 3. A Banach module over $M_{0}$ which is not normal. Let $T^{1}$ be the circle group $R / 2 \pi Z$ and $Z$ the dual group of $T^{1}$ (the integers). Let $\left\{Z_{k}\right\}$ be a countable set of disjoint infinite classes such that $Z=\cup^{\infty} Z_{k}$. For every $k$, let $I_{k}$ be the closed ideal in $L^{1}$ such that $f \in I_{k}$ iff $\hat{f}(n)=0 \forall n \in Z_{k}$.

Define, for every $k, L_{k}^{1}=L^{1} / I_{k}, H_{k}^{1}=\hat{H} / I_{k}$, where $\hat{H}$ is the Banach space of Example 1.

It is clear that $L_{k}$ and $H_{k}^{1}$ correspond to spaces of pseudomeasures such that $\hat{\mu}(n)=0 \forall n \notin Z_{k}$. Therefore $L_{k}^{1}$ and $H_{k}^{1}$ may be identified with spaces of distributions. Furthermore since $\hat{A} \cap L^{1}=\{0\}$ it follows easily that as spaces of distributions $L_{k}^{1} \cap H_{k}^{1}=\{0\}$ for every $k$.

Let $X_{0}$ be the class of all finite linear combinations $X_{0}=\left\{\Sigma^{N} \alpha_{k} D^{k} f_{k}, f_{k} \in L_{k}^{1}\right\}$ where $D^{k}$ is the $k$ th distributional derivative of $f_{k}$.

$X_{0}$ may be endowed with the norm

$$
\|\lambda\|_{\mathbb{R}_{0}}=\inf \stackrel{N}{\Sigma}\left|\alpha_{k}\right|\left\|f_{k}\right\|_{h}
$$

where the inf in (4.2) is taken with respect to all finite sums such that $\lambda=\Sigma^{N} \alpha_{k} D^{k} f_{k}$. Let $X_{0}$ be the completion of $X_{0}$ under the norm (4.2).

Let $H_{1}$ be the class of all finite linear combinations $H_{1}=\left\{\Sigma^{N} \alpha_{k} D^{k} \mu_{k} \mid \mu_{k}\right.$ $\left.\in H_{k}\right\} . H_{1}$ may be endowed with the norm

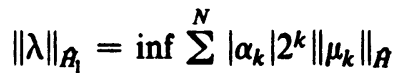

where again the inf is taken over all sums such that $\lambda=\Sigma^{N} \alpha_{k} D^{k} \mu_{k}$.

It is clear that for every $k, D^{k} H_{k} \cap D^{k} L_{k}=\{0\}$ since if $D^{k} \mu_{k}=D^{k} f_{k}$, it follows that $\mu_{k}=f_{k}+g$ (where $\left.g \in L^{L}\right)$. This is impossible since $H_{k} \cap L_{k}=\{0\}$. Hence it follows that $\hat{A}_{1} \cap \bar{X}_{0}=\{0\}$.

Therefore one may define $X=\hat{H}^{1} \oplus \hat{X}_{0}$. 
It is clear that $X$ has norm invariant with respect to translation since both the norms of $\hat{H}^{1}$ and $\hat{X}_{0}$ are invariant under translation. Furthermore it is clear that for every $h \in L^{1}$ and $\lambda \in H$,

$$
h * \lambda=\sum^{N} \alpha_{k} h * D^{k} \mu_{k}=\sum^{N} \alpha_{k} D^{k}\left(h * \mu_{k}\right) \in X_{0}
$$

since $h * \mu_{k} \in L_{k}^{1}$ for every $h \in L^{1}$. Furthermore

$$
\begin{aligned}
\|h * \lambda\|_{\delta_{0}} & \leq \inf \sum^{N}\left|\alpha_{k}\right|\left\|h * \mu_{k}\right\|_{h} \leq \inf \left(\sum^{N}\left|\alpha_{k}\right|\left\|\mu_{k}\right\|_{h}\right)\|h\|_{h} \\
& \leq\left(\inf \sum^{N}\left|\alpha_{k}\right| 2^{k}\left\|\mu_{k}\right\|_{h}\right)\|h\|_{h}=\|\lambda\|_{R_{1}}\|h\|_{h} .
\end{aligned}
$$

Since clearly $h * \lambda \in \hat{X}_{0}$ for every $\lambda \in \hat{X}_{0}$, it follows that $X=\hat{H}^{\prime} \oplus \hat{X}_{0}$ is a Banach module over $M_{0}$.

However $X$ is not normal. Let $\left\langle\mu_{k}\right\rangle$ be the sequence of pseudomeasures defined by

$$
\begin{array}{ll}
\hat{\mu}_{k}(n)=\hat{\delta}_{0}(n) & \text { for } n \in Z_{k}, \\
\hat{\mu}_{k}(n)=0 & \text { for } n \neq Z_{k} .
\end{array}
$$

Consider the sequence $\left\langle D^{k} \mu_{k}\right\rangle$ in $\hat{H}^{1}$. It is then easy to see that

$$
\left\|D^{k} \mu_{k}\right\|_{x}^{\prime}=\sup _{\|h\|_{1} \leq 1}\left\|h * D^{k} \mu_{k}\right\|_{x}=\left\|\mu_{k}\right\|_{h}
$$

while $\left\|D^{k} \mu_{k}\right\|_{x}=2^{k}\left\|\mu_{k}\right\|$ by (4.2).

Hence the norms $\|\cdot\|_{X}^{\prime}$ and $\|\cdot\|_{X}$ are clearly not equivalent.

By the uses of methods developed by Rieffel in [30] and [31], it is also possible to characterize the spaces of convolution multipliers $[X, Y]$ for a large class of Banach modules $X, Y \in M_{00}$. The following theorem is fundamental to such a characterization.

Theorem 4.1 (Rieffel [30]). Let $X$ and $Y$ be Banach modules over a ring $R$. Then there exists an isometric isomorphism

$$
\left[X, Y^{*}\right] \cong\left(X \otimes_{R} Y\right)^{*} .
$$

Theorem 4.1 is not difficult to prove; it follows easily from Definition 1.1. Grothendieck observed long ago in [13] that (4.3) could be used to characterize the space of linear operators from $X$ to $Y^{*}$ when $R$ is the ring of real or complex numbers. The possibility of using (4.3) to characterize general module homomorphisms seems to have first been observed by Rieffel [30]. In [31], Rieffel employs this theorem together with methods developed by Figa-Talamanca and Gaudry [12] to obtain a characterization as a concrete function space of the space of 
multipliers $\left(L^{p}, L^{q}\right)$ over a locally compact group $G$. A generalization of his method to arbitrary Banach spaces of functions allows one to prove Theorem 4.2 below.

Let $T^{n}$ be a toroidal group and $\mathcal{D}_{c}$ the category of Banach modules over $M_{0}$ of complex-valued measures on $T^{n}$, such that the translation operator $\delta_{x} f$ forms a continuous map from $T^{n}$ to $X$ for every $x \in T^{n}$ and $f \in X$.

It is clear that if $X \in M_{c}$ and $f \in X$, then $f$ may be approximated by a $C^{\infty}$ function in $X$; for if $\left\langle\varphi_{k}\right\rangle \subseteq M_{0}$ is a $C^{\infty}$ approximate identity, the continuity of translations in $X$ implies that $\varphi_{k} * f \rightarrow f$. It therefore follows that $X^{*}$ is a space of distributions in $D$ for every $X \in \mathcal{X}$.

It is furthermore clear that if $f$ is in $L^{1}$ for every $f \in X$, then $X$ is embedded continuously in $M_{0}$.

Definition 4.1. For every $X, Y \in \mathcal{C} \mu_{c}$ let $B$ denote the linear operator $B: X \otimes Y^{*} \rightarrow D_{n}$ defined by $B(f \otimes g)=\tilde{f} * g$ for every $f \in X$ and $g \in Y^{*}$ and uniquely defined on all of $X \otimes_{M} Y^{*}$ by linearity. Let $A(X, Y)$ denote the range of $B$, endowed with the induced norm from $X \otimes Y^{*}$.

Clearly $A(X, Y)$ becomes a Banach space under the given norm; clearly $A(X, Y)$ will consist of exactly those distributions $h$ which may be written in at least one way as $h=\sum^{\infty} \tilde{f}_{i} * g_{i}$ with $f_{i} \in X$ and $g_{i} \in Y^{*}$ for every $i$.

It is now possible to prove the following general representation theorem.

Theorem 4.2. Let $T^{n}$ be a toroidal group and $X, Y \in \mathcal{C} M_{c}$. Then there exists an isometric isomorphism $X \otimes_{M_{0}} Y^{*} \cong A(X, Y)$.

Proof. Let $K$ be the closed subspace of $X \otimes Y^{*}$ spanned by elements of the form $(\mu * f) \otimes g-f \otimes(\bar{\mu} * g)$, where $\mu \in M, f \in X$, and $g \in Y^{*}$. It will be sufficient, to show the theorem, to show that the kernel of $B$ is exactly $k$.

It is first clear that $K \subseteq \operatorname{ker} B$, since

$$
B((\mu * f) \otimes g)=(\mu * f)^{\sim} * g=\tilde{f} *(\bar{\mu} * g)=B(f \otimes(\bar{\mu} * g)) .
$$

To show the converse, let $h \in \operatorname{ker} B$; it must be shown that $h \in K$. Let $h=\Sigma^{\infty} f_{i} \otimes g_{i}$ be an expansion for $h \in X \otimes Y^{*}$. Since $h \in \operatorname{ker} B, B(h)=$ $\sum \tilde{f}_{i} * g_{i}=0$, where the sum converges absolutely in the norm of $A(X, Y)$.

I shall now construct a sequence $\left\langle\varphi_{k}^{\prime}\right\rangle$ of $C^{\infty}$ functions such that $\left\langle\varphi_{k}^{\prime}\right\rangle$ acts as an approximate identity on the space $X$. Let $\left\langle\varphi_{k}\right\rangle$ be an approximate identity in $M_{0}$ consisting of linear combinations $\sum_{|m|_{2} \leq k} \alpha_{m} e_{m}=\varphi_{k}$ for every $k$. Let $Z_{X}$ $=\left\{m \in Z^{n} \mid \exists f \in X \ni f(m) \neq 0\right\}$, and define a sequence of distributions $\left\langle\delta_{k}\right\rangle$ such that

$$
\begin{array}{ll}
\hat{\delta}_{k}(m)=1 & \text { for all } m \in Z_{X} \ni|m|_{2} \leq k, \\
\hat{\delta}_{k}(m)=0 & \text { otherwise. }
\end{array}
$$

Finally define $\varphi_{k}^{\prime}=\varphi_{k} * \delta_{k}$. $\varphi_{k}$ is clearly well defined in the sense of distributions for every $k$; furthermore for every $f \in X, \varphi_{k}^{\prime} * f=\varphi_{k} * \delta_{k} * f=\varphi_{k} * f$ by the 
construction (4.4) for $\delta_{k}$ and the definition of $\varphi_{k}=\sum_{|m|_{2} \leq k} \alpha_{m} e_{m}$. Hence $\varphi_{k}^{\prime} * f$ $\rightarrow f$ in the strong topology of $X$.

For each $k$, define $h_{k} \in X \otimes Y^{*}$ by $h_{k}=\sum_{i=1}^{\infty}\left(f_{i} * \varphi_{k}^{\prime}\right) \otimes g_{i}$.

Since $f_{i} * \varphi_{k}^{\prime} \rightarrow f_{i}$ for every $i$ and $h$ converges absolutely, it follows that $h_{k} \rightarrow h$. Since $K$ is closed it will be sufficient to show that $h_{k} \in K$ for every integer $k$.

Since $\sum f_{i} * \tilde{g}_{i}=0$, for every $k$ and $\varepsilon>0$, there exists $m_{0}$ such that, for every $m>m_{0}$,

$$
\left\|\sum_{i=1}^{m} f_{i} * g_{i}\right\|_{A(X, Y)}<\frac{\varepsilon}{2}\left\|\varphi_{k}^{\prime}\right\|_{X}
$$

Again since $h_{k} \rightarrow h$, there exists $m_{1}$ such that, for $m>m_{1}$,

$$
\left\|h_{k}-\sum^{m}\left(f_{i} * \varphi_{k}^{\prime}\right) \otimes g_{i}\right\|<\frac{\varepsilon}{2} \text {. }
$$

One may now observe that

$$
\begin{aligned}
\sum^{m}\left(f_{i} * \varphi_{k}^{\prime}\right) \otimes g_{i}= & \sum^{m} \varphi_{k}^{\prime} \otimes\left(\tilde{f} * g_{i}\right) \\
& +\sum^{m}\left[\left(f_{i} * \varphi_{k}\right) \otimes g_{i}-\varphi_{k} \otimes\left(\tilde{f}_{i} * g_{i}\right)\right] \\
= & h_{1}^{k}+h_{2}^{k} .
\end{aligned}
$$

Clearly $h_{2}^{k} \in K$ since $\varphi_{k}^{\prime} \in X$ for every $k$. However if $m>m_{1}$, then

$$
\left\|\sum^{m} \varphi_{k}^{\prime} \otimes\left(\tilde{f}_{i} * g_{i}\right)\right\|=\left\|\varphi_{k}^{\prime}\right\|_{X}\left\|\sum^{m} \tilde{f}_{i} * g_{i}\right\|_{Y^{*}} .
$$

Since $X \subseteq M_{0}$ for every $X \in M_{0}, f_{i} \in M_{0}$; since $Y$ is a Banach module over $M_{0}$, and convolution is commutative, it therefore follows that $Y^{*}$ is a Banach module over $M_{0}$ by duality. It therefore follows that there exists a constant $K$ such that for every $m$

$$
\left\|\sum \tilde{f}_{i} * g_{i}\right\|_{Y^{*}} \leq K\left\|\Sigma \tilde{f}_{i} * g_{i}\right\|_{A(X, Y)}
$$

and therefore $\left\|\Sigma^{m} \varphi_{k}^{\prime} \otimes\left(\tilde{f}_{i} * g_{i}\right)\right\| \leq\left\|\varphi_{k}^{\prime}\right\|_{X}\left\|\Sigma^{m} f_{i} * g_{i}\right\|_{A} \leq \varepsilon / 2$ where the last inequality follows from (4.5). Combining (4.5) with (4.8) it follows that $\left\|h_{1}^{k}\right\|$ $<\varepsilon / 2$. Since $\varepsilon$ is arbitrary it follows from (4.7) that $h \in K$.

It follows from Theorem 4.2 that if $X$ and $Y$ are Banach modules over $M$ in the category $M_{c}$, then $\left[X, Y^{* *}\right]=\left(X \otimes_{M} Y^{*}\right)^{*}$. However it is also true that if $X$, $Y \in M_{c}$, then $[X, Y]=\left[X, Y^{* *}\right]$.

Proposition 4.1. Let $X$ and $Y$ be Banach modules in the category o $M_{c}$. Then $[X, Y]=\left[X, Y^{* *}\right]$. 
Proof. Clearly $[X, Y] \subseteq\left[X, Y^{* *}\right]$. To show the contrary, let $H \in\left[X, Y^{* *}\right]$. It will clearly be sufficient to show that if $p$ is a trigonometric polynomial then $H p \in Y$. It will then follow since the trigonometric polynomials are dense in $X$, that $H: X \rightarrow Y$.

Lemma 4.1.1. Let $H \in\left[X, Y^{* *}\right]$ where $X$ and $Y$ are in the category $u_{c} ;$ suppose that $p$ is a trigonometric polynomial. Then $H: X \rightarrow L$ and $H$ is a convolution multiplier.

Proof. If $Y$ is a Banach space in $L^{1}$ it follows that $Y^{* *} \subseteq\left(L^{1}\right)^{* *}=\left(L^{\infty}\right)^{*}$.

Let $f \in L^{\infty}$ and $\mu \in\left(L^{\infty}\right)^{*}=\left(L^{1}\right)^{* *}$. $\left(L^{\infty}\right)^{*}$ is the class of additive Borel measures, not necessarily regular, on $T^{n}$. Define a convolution of $f \in L^{\infty}$ and $\mu \in\left(L^{\infty}\right)^{*}$ by

$$
f * \mu(x)=\left\langle\check{f}_{x}, \mu\right\rangle=\int f(x-t) d \mu_{t}
$$

where $\breve{f}(t)=f(-t)$. Clearly $f * \mu$ is measurable and well defined as a bilinear mapping from $L^{\infty} \times\left(L^{\infty}\right)^{*}$ to $L^{\infty}$.

I shall show that this convolution product may be extended to a bilinear mapping from $L^{1} \times\left(L^{\infty}\right)^{*}$ to $\left(L^{\infty}\right)^{*}$. Let $g \in L^{\infty}, f \in L^{\infty}$, and $\mu \in\left(L^{\infty}\right)^{*}$. Then

$$
\langle g, f * \mu\rangle=\langle g * \tilde{f}, \mu\rangle \leq\|\tilde{f}\|_{1}\left\|_{g}\right\|_{\infty}\|\mu\|_{*}=\|f\|_{h}\left\|_{g}\right\|_{\infty}\|\mu\|_{*} .
$$

Therefore for all $f \in L^{\infty}$ and $\mu \in\left(L^{\infty}\right)^{*}$

$$
\|f * \mu\|_{\left(L^{\infty}\right)^{*}} \leq\|f\|_{1}\|\mu\|_{\left(L^{\infty}\right)^{\bullet}}
$$

Since $L^{\infty}$ is dense in $L^{1}$, the convolution defined by (4.10) is well defined as a bilinear operator from $L^{1} \times\left(L^{\infty}\right)^{*}$ to $\left(L^{\infty}\right)^{*}$, and hence as a bilinear operator from $L^{1} \times\left(L^{1}\right)^{* *}$ to $\left(L^{1}\right)^{* *}$.

Let $p$ be a trigonometric polynomial in $X$ and $\mu=H p$. Since $H$ commutes with $M_{0}, H$ commutes with translations; it therefore follows that $\left\|\left(\mu_{x}-\mu\right) / x\right\|_{Y 00}$ is bounded as $x \rightarrow 0$, and hence that $\left\|\left(\mu_{x}-\mu\right) / x\right\|_{(D) * *}$ is bounded since $Y^{* *}$ $\subseteq\left(L^{1}\right)^{* *}$.

Let $\left\langle\varphi_{k}\right\rangle$ be an approximate identity of norm 1 in $L$, such that $\varphi_{k} \in L^{\infty}$ for every $k$. By the inequality (4.11) and the fact that $\left.\left\|\mu_{x}-\mu\right\|_{(L)}\right)^{* *} \leq c \cdot x$, it follows that $H\left(\varphi_{k} * p\right)=\varphi_{k} * H p \rightarrow H p$ as $k \rightarrow \infty$ in the strong $\left(L^{1}\right)^{* *}$ norm. But since $\varphi_{k} \in L^{\infty},(4.11)$ implies that $\varphi_{k} * H p \in L^{\infty} \subseteq L^{1}$ for every $k$. Since $L^{1}$ is closed in $\left(L^{1}\right)^{* *}$, it follows that $H p \in L$. Since the trigonometric polynomials are dense in $X$, it follows that $H: X \rightarrow L$, and by Proposition 4.0 that $H$ is a convolution multiplier.

The second lemma will complete the proof of Proposition 4.1.

Lemma 4.1.2. Let $H, X$, and $Y$ be as in the statement of Lemma 4.1.1 and let $p$ be a trigonometric polynomial. Then $H p \in Y$. 
Proof. Let $Z_{Y^{* *}}=\left\{m \in Z^{n} \mid \exists f \in Y^{* *} \cap L^{1} \ni \hat{f}(m) \neq 0\right\}, Z_{Y}=\left\{m \in Z^{n} \mid\right.$ $\exists f \in Y \ni \hat{f}(m) \neq 0\}$. Clearly $Z_{Y} \subseteq Z_{Y * *}$. I shall show that $Z_{Y * *}=Z_{Y}$. Since $H$ is a convolution multiplier by Lemma 4.1.1 it will follow that $H p \in Y$ for every trigonometric polynomial $p$.

Let $y^{* *} \in Y^{* *}$. Then $y^{* *}$ defines a linear functional on $Y^{*}$. Since the trigonometric polynomials are dense in $Y$, every $y^{*} \in Y^{*}$ may be identified with the equivalence class

$$
j\left(y^{*}\right)=\left\{\lambda \in D \mid \hat{\lambda}(m)=\left\langle\lambda, e_{m}\right\rangle=\left\langle y^{*}, e_{m}\right\rangle \forall m \in Z_{Y}\right\} .
$$

Since $j$ is a projection it follows that $j^{*}$ is an injection; hence for every $y^{* *}$ and $y^{*}$

$$
\left\langle y^{* *}, j\left(y^{*}\right)\right\rangle=\left\langle j^{*}\left(y^{* *}\right), y^{*}\right\rangle=\left\langle y^{* *}, y^{*}\right\rangle \text {. }
$$

Suppose that $m_{0} \in Z_{Y^{* *}}-Z_{Y}$. Then $e_{m_{0}} \in Y^{* *}$ and therefore $\left\langle e_{m_{0}}, y^{*}\right\rangle$ $=\left\langle e_{m_{0}}, j\left(y^{*}\right)\right\rangle$ for every $y^{*} \in Y^{*}$. Hence by (4.11), $\left\langle e_{m_{0}}, e_{m}\right\rangle=0$, for every $m \notin Z_{Y}$, and therefore $\left\langle e_{m_{0}}, e_{m_{0}}\right\rangle=0$. This is a contradiction; hence $Z_{Y} \cdot Z_{Y}$ and the lemma is proved.

From Lemma 4.1.1 it follows easily that $H: X \rightarrow Y$, since the polynomials $p$ are dense in $X$.

Using Theorems 4.1 and 4.2, and Proposition 4.1 the next theorem may be easily obtained.

Theorem 4.3. Let $T^{n}$ be a toroidal group and $X, Y \in M_{00}$. Then there exists an isometric isomorphism $[X, Y] \cong A\left(X, Y^{*}\right)^{*}$.

2. Tensor multiplier problems for Banach module functors. In this section I shall discuss the tensor multiplier functors for $L^{\infty}, C$, and $C^{r}$.

I shall first discuss the tensor multiplier functors $\operatorname{Hom}_{\otimes}[C, C]$ and $\operatorname{Hom}_{\circledast}\left[L^{\infty}, L^{\infty}\right]$. As in the case of complex-valued convolution multipliers $[C, C]$ and $\left[L^{\infty}, L^{\infty}\right]$ it is possible to obtain a complete characterization of the tensor multiplier functors.

Theorem 4.4. Let $\mathcal{B}$ be the category of Banach spaces and $T^{n}$ a toroidal group. Let $M$ be the measure functor on $T^{n}, C$ the continuity functor, and $L^{\infty}$ the $L^{\infty}$ functor from $\mathcal{B}$ to $\boldsymbol{N}$. Then

$$
M \cong \operatorname{Hom}_{\otimes}[C, C] \cong \operatorname{Hom}_{\otimes}\left[L^{\infty}, L^{\infty}\right]
$$

Proof. I shall prove the theorem by showing successively that

$$
M \cong \operatorname{Hom}_{\otimes}[C, C] \text { and } M \cong \operatorname{Hom}_{\otimes}\left[L^{\infty}, L^{\infty}\right]
$$

For the first part, I shall first prove a lemma.

Lemma 4.4.1. Let $\mathcal{B}$ be the category of Banach spaces and $T^{n}$ a toroidal group. Let $C$ be the continuity functor on $\mathcal{B}$ and $T^{n}$, and $D C$ the dual functor to $C$. Then 
there exists an isometric isomorphism $M \cong i$

$$
i_{Y}(\mu)(f)=\int f(t) \otimes d \mu
$$

for every $X \in \mathcal{B}, f \in C(X)$, and $\mu \in M(Y)$.

Proof. Lemma 4.4.1 is proved in less general form by Mityagin and Švarc in [24]. The proof given here is an adaptation of their proof.

In [13, Proposition 32, pp. 152-153], Grothendieck proves the following equivalence. Let $E$ and $F$ be Banach spaces and $A$ a linear operator $A: E \rightarrow F$. Then the statement (1) below holds if and only if (2) holds.

(1) There exists a constant $M<\infty$ such that for every collection of elements $\left\{e_{k}\right\}_{k=1}^{N} \leq E$,

$$
\sum^{N}\left\|A e_{k}\right\|_{F} \leq M \sup \left\{\left\|\xi^{N} \xi_{k} e_{k}\right\|_{E}|| \xi_{k} \mid \leq 1, k=1, \ldots, N\right\} .
$$

(2) For every unconditionally convergent series $\Sigma^{\infty} e_{k}$ in $E$, the series $\Sigma^{\infty} A e_{k}$ converges absolutely.

For the case of operators $v: C \rightarrow Y$, the first condition is just the condition that $\lambda \in M(Y)$.

Now let $Y \in \mathcal{B}$ and $\alpha \in D C(Y)=\left[C, \Sigma_{Y}\right]$, and let $\nu$ be the mapping $\nu: D C \rightarrow \Omega_{C}$ given by $\nu_{Y}(\alpha)=\alpha_{C} \forall Y \in \mathcal{B}$. Then $\nu_{Y}(\alpha)=\alpha_{C}$ defines an operator $\alpha_{C}: C \rightarrow Y$. To show that $\alpha_{C} \in M(Y)$, let $\sum g_{k}$ be an unconditionally convergent series in $C$. Let $y_{k}=\alpha_{c}\left(g_{k}\right)$, and define a sequence $\left\langle y_{k}^{*}\right\rangle \subseteq Y^{*}$ such that $\left\|y_{k}^{*}\right\|=1$ and $\left\langle y_{k}^{*}, y_{k}\right\rangle \mid=\left\|y_{k}\right\|$.

Since the series $\Sigma^{N} \xi_{k} g_{k}$ converges in $C$ for any set of numbers $\left\{\xi_{k}\right\}$ with $\left|\xi_{k}\right| \leq 1$, and the sums are uniformly bounded, $\Sigma^{N}\left|g_{k}\right| \leq B<\infty$ uniformly for every $N$ and $t$. Hence the sequence of functions $\left\langle G_{N}\right\rangle$ defined by

$$
G_{N}(t)=\sum^{N} g_{k}(t) y_{k}^{*} \text { for } t \in T^{n}
$$

is uniformly bounded.

For every $N, \Sigma^{N} g_{k} \cdot y_{k}^{*}$ is contained in the image of the mapping $i_{\gamma_{\bullet}}$ : $C \otimes Y^{*} \rightarrow C\left(Y^{*}\right)$. Therefore it follows by Proposition 1.4 that $\alpha_{*}\left(G_{N}\right)=$ $\Sigma^{N} \alpha_{c}\left(g_{k}\right) \otimes y_{k}^{*}=\Sigma^{N} y_{k} \otimes y_{k}^{*}$. Therefore

$$
\begin{aligned}
\sum^{N}\left\|\alpha_{C}\left(g_{k}\right)\right\| & =\sum^{N}\left\|y_{k}\right\|=\sum^{N}\left\langle y_{k}^{*}, y_{k}\right\rangle \leq\left\|\alpha_{Y^{\bullet}}\left(G_{N}\right)\right\|_{Y \otimes Y^{\bullet}} \\
& \leq\left\|\alpha_{Y^{\bullet}}\right\|\left\|G_{N}\right\|_{C\left(Y^{\bullet}\right)} \leq\|\alpha\|_{D C(Y)} \cdot B .
\end{aligned}
$$

Hence $\nu_{Y}(\alpha)=\alpha_{C} \in M(Y)$.

To show that the mapping $\nu_{Y}$ is onto, let $\alpha_{0} \in M(Y)$ and define an operator $\alpha_{X}: C(X) \rightarrow Y \otimes X$ as follows: let $f \in C(X)$. Since $f: T^{n} \rightarrow X$ is a continuous function on the compact space $T^{n}$, for every $\varepsilon>0$ there exists 
(1) a finite covering of $T^{n}$ by open sets $\left\{U_{k}\right\}_{k=1}^{N}$,

(2) a partition of unity $\left\{\varphi_{k}\right\}$ subordinate to $\left\{U_{k}\right\}_{n}$,

(3) elements $x_{k} \in X, k=1, \ldots, N$, such that

$$
\left\|f-\sum^{N} x_{k} \varphi_{k}\right\|_{C(X)}<\varepsilon
$$

Define

$$
\alpha_{X}\left(\sum^{N} x_{k} \varphi_{k}\right)=\sum^{N} \alpha_{0}\left(\varphi_{k}\right) \otimes x_{k} .
$$

Since $\alpha_{0} \in M(Y)$, it follows that (see Definition 1.8)

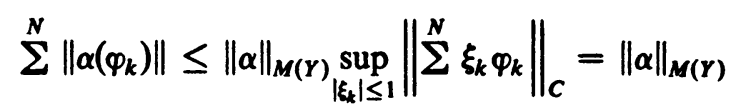

and

$$
\left\|\alpha_{X}\left(\sum^{N} x_{k} \varphi_{k}\right)\right\| \leq \sum^{N}\left\|\alpha_{0}\left(\varphi_{k}\right)\right\|\left\|x_{k}\right\| \leq\|f\|_{C(X)}\|\alpha\|_{M(Y)} .
$$

Since, by (4.18), $\alpha_{X}$ is a continuous operator $\alpha_{X}: C(X) \rightarrow Y \otimes X, \alpha_{X}$ may be extended by continuity to the whole space $C(X)$.

It is clear from the representation (4.17) that $\nu_{Y}(\alpha)=\alpha_{C}=\alpha_{0}$.

To show that the mapping $v: D C \rightarrow M$ is an isometry, observe that, by (4.15), $\|\alpha\|_{M(Y)} \leq\|\alpha\|_{\infty C(Y)}$ and, by (4.18), $\|\alpha\|_{\infty C(Y)} \leq\|\alpha\|_{M(Y)}$. To show that the mapping $\nu: D C \rightarrow M$ is natural, observe that the mapping $\nu: D C \rightarrow M^{*}=\Omega_{C}$ is natural since the diagram

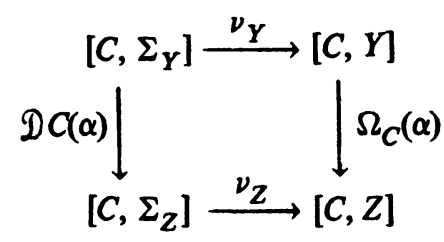

commutes; $\nu_{Z} D C(\alpha)\left(\lambda_{x}\right)=\nu_{Z} \circ\left(\alpha \otimes 1_{X}\right) \circ \lambda_{X}=c \circ \lambda_{C}=\Omega_{Z}(\alpha) \nu_{Y}\left(\lambda_{x}\right)$. Hence $\nu: D C \rightarrow M$ is also natural since $M$ is a norm subfunctor of $M^{*}$.

To obtain the representation (4.14) it is only necessary to define $i=\nu^{-1}$. The representation (4.14) then follows from the Dunford-Schwartz representation theorem (Lemma 1.0).

It is now possible to proceed with the proof of Theorem 4.4.

To prove that $\operatorname{Hom}_{\otimes}[C, C] \cong M$, let $h \in \operatorname{Hom}_{\otimes}[C, C]$. Then for every $X$ and $Y$ in $\mathcal{B}$, and $f \in C(X), h_{X}(f) \in C(Y \otimes X)$. Let $\check{f}(t)=f(-t)$. Then for $t=0$, $h_{X}(\check{f})(0) \in Y \otimes X=\Sigma_{Y}(X)$. The mapping 


$$
\delta_{0}^{Y}\left(h_{X}(\check{f})\right)=h_{X}(\check{f})(0)
$$

therefore defines a monomorphism $\delta_{0}^{Y}: \operatorname{Hom}_{\otimes}[C, C](Y) \rightarrow D C(Y)$ and hence every $h \in \operatorname{Hom}_{\otimes}[C, C](Y)$ defines a mapping $\delta_{0}(h) \in D C(Y) \cong M(Y)$. Conversely, let $\mu \in M(Y)$ and define a mapping $\tau_{Y}: M(Y) \rightarrow \operatorname{Hom}_{\circledast}[C, C](Y)$ by

$$
\tau_{Y}(\mu)(f)(s)=\int \check{f}_{s} \otimes d \mu
$$

for every $X \in \mathcal{B}$ and $f \in C(X)$. Since $\mu \in M(Y) \cong D C(Y)$, by the isomorphism of Lemma 4.4.1 it follows at once that

$$
\begin{aligned}
\left\|\tau_{Y}(\mu)(f)\right\|_{C(X \otimes Y)} & =\sup _{s}\left\|\tau_{Y}(\mu)(f)(s)\right\|_{X \otimes Y} \leq \sup _{s}\left\|\int \check{f}_{s} \otimes d \mu\right\|_{X \otimes Y} \\
& \leq \sup _{s}\left\|\tau_{X}(\mu)\right\|\left\|\check{f}_{s}\right\|_{X}=\|\mu \mu\| \cdot\|f\|_{C(X)} .
\end{aligned}
$$

Since $C: B \rightarrow \mathcal{B}$, it follows that the homomorphism $h_{X} \in[C(X), C(Y \otimes X)]$ commutes with translations for every $X$.

Let $i_{Y}$ be the isomorphism (4.14) and let $\mu_{(h, X)}=i_{Y} \circ \delta_{0}^{Y}\left(h_{X}\right)$ for $h_{X} \in[C(X)$, $C(Y \otimes X)]$. Since $C: \mathcal{B} \rightarrow \mathcal{O}$, it follows that the homomorphism $h_{X}$ commutes with translations for every $X$. Hence

$$
h_{X}(\check{f})(s)=\delta_{0}^{Y}\left(\left[h_{X}(\check{f})\right]_{s}\right)=\delta_{0}^{Y}\left(h_{X}\left(\check{f}_{s}\right)\right)=\int \check{f}_{s} \otimes d \mu_{(h, X)} .
$$

Therefore $\tau_{Y}$ and $\delta_{0}^{Y}$ are inverses.

To see that the map $\tau$ defined by $\tau_{X}$ in (4.20) for every $Y$ defines an isomorphism $\tau: M \cong \operatorname{Hom}_{\otimes}[C, C]$, it is now only necessary to show that the diagram

$$
\begin{gathered}
M(Y) \stackrel{\tau_{Y}}{\longrightarrow} \operatorname{Hom}_{\otimes}[C, C](Y) \\
\qquad(\alpha) \downarrow \\
M(Z) \underset{\tau_{Z}}{\longrightarrow} \operatorname{Hom}_{\otimes}[C, C](Z)
\end{gathered}
$$

commutes; this follows at once since for every $X \in \mathcal{B}, f \in C(X), h \in$ $\mathrm{Hom}_{\circledast}[C, C]$, and $\alpha \in[Y, Z]$,

$$
\begin{aligned}
\operatorname{Hom}_{\otimes}[C, C](\alpha) \tau_{Y}(\mu)(f) & =C\left(1_{X} \otimes \alpha\right) \int \check{f}_{s} \otimes d \mu \\
& =\int \check{f}_{s} \otimes \alpha d \mu=\tau_{Y}(M(\alpha) \mu)(f) .
\end{aligned}
$$

I shall now show that $\operatorname{Hom}_{\otimes}\left[L^{\infty}, L^{\infty}\right] \cong M$. 
Define a mapping $\sigma: \operatorname{Hom}_{\odot}\left[L^{\infty}, L^{\infty}\right] \rightarrow \operatorname{Hom}_{\odot}[C, C]$ by $\sigma_{Y}\left(h_{X}\right)=\left.h_{X}\right|_{C(X)}$, the restriction of $h$ to the functor $C$. $\sigma$ defines a homomorphism, since if $h$ $\in \operatorname{Hom}_{\otimes}\left[L^{\infty}, L^{\infty}\right]$,

$$
\left\|h_{X}\left(f_{s}-f\right)\right\|_{L^{\infty}(X \otimes Y)} \leq\|h\|\left\|f_{s}-f\right\|_{L^{\infty}(X)}
$$

for every translation $f_{s}$ of $f$ for $s \in T^{n}$. Hence for every $f \in C(X), h_{X}(f)$ $\in C(X \otimes Y)$. Since $C$ is a subfunctor of $L^{\infty}$ it is clear that $\sigma: \operatorname{Hom}_{\circledast}\left[L^{\infty}, L^{\infty}\right]$ $\rightarrow \operatorname{Hom}_{\circledast}[C, C]$ is a natural mapping.

To show that $\sigma$ is a monomorphism, suppose first that $\sigma_{Y}\left(h_{C}\right)(f)=h_{C}(f)=0$ for every continuous $f \in C$. By Proposition 4.0, $h_{\mathrm{C}}(f)=h_{0} * f$ for some $Y$ valued distribution $h_{0}$; since $h_{\mathrm{C}}(f)=0$ for every continuous $f, h_{0}$ is trivial. Therefore $h_{\mathbf{C}}$ is trivial.

Suppose that $h_{\mathrm{C}}$ is trivial; then to show that $h_{X}$ is trivial for every $X \in \mathcal{B}$, observe that $h$ is a natural mapping $h \in\left[L^{\infty}, L^{\infty} \Sigma_{Y}\right]$. Therefore the diagram

$$
L^{\infty} \stackrel{L^{\infty}(\alpha)}{\longrightarrow} L^{\infty}(X)
$$

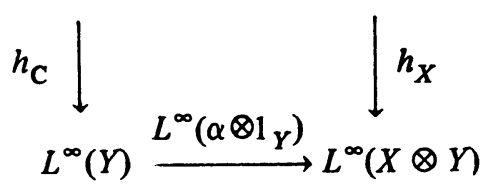

commutes for all $\alpha: \mathbf{C} \rightarrow X$ and $X, Y \in \mathcal{B}$. Let $\lambda \in L^{\infty}(X)$ and $\hat{\lambda}_{m}$ be the Fourier transform of $\lambda$ at $m$. Then since $h$ is a module homomorphism over $M$,

$$
h_{X}(\lambda) * e_{m}=h_{X}\left(\lambda * e_{m}\right)=h_{X}\left(\hat{\lambda}_{m} e_{m}\right) .
$$

Let $k\left(\hat{\lambda}_{m}\right): \mathbf{C} \rightarrow X$ be the mapping defined by $K\left(\hat{\lambda}_{m}\right)(1)=\lambda_{m}$. Then by the commutativity of the diagram (4.21) and the fact that $h_{\mathrm{C}}$ is trivial

$$
h_{X}\left(\hat{\lambda}_{m} e_{m}\right)=\hat{\lambda}_{m} \otimes h_{\mathrm{c}}\left(e_{m}\right)=0
$$

for every $m$. Hence $h_{X}(\lambda) * e_{m}=0$ for every $m$. Therefore $h_{X}$ is trivial.

Define a mapping $\gamma: \operatorname{Hom}_{\circledast}\left[L^{\infty}, L^{\infty}\right] \rightarrow M$ by

$$
\gamma_{Y}(h)=i_{Y} \circ \delta^{Y} \circ \sigma_{Y}(h)
$$

where $i_{Y}$ is the isomorphism given by (4.14), $\delta^{Y}$ is the isomorphism defined by (4.19) and $\sigma_{Y}$ is the isomorphism defined above. Since $\gamma$ is the composition of natural mappings, $\gamma$ must itself be a natural mapping. It therefore remains only to show that the mapping $\gamma$ is onto.

This will be done and the theorem completed in a lemma:

Lemma 4.4.2. Let $\gamma: \operatorname{Hom}_{\otimes}\left[L^{\infty}, L^{\infty}\right] \rightarrow M$ be defined by (4.22). Then $\gamma$ is onto all of $M$. 
Proof. Let $Y \in \mathcal{B}$ and $\mu \in M(Y)$. Consider the operator $M_{f}: C \rightarrow Y$ defined by $M_{f}(g)=\int\left[\int \hat{f}_{s} d \mu\right] g(s) d s$. $M_{f}$ is well defined for every $f \in L^{\prime}$ and $g \in C$. Furthermore since $\mu * f=\int \hat{f}_{s} d \mu$ is continuous in $f$, it follows that

$$
M_{f}(g)=\int\left[\int \hat{f}_{s} d \mu\right] g(s) d s=\int\left[\int \hat{f}_{s} g(s) d s\right] d \mu .
$$

Since $M_{f}: C \rightarrow Y$, by Lemma 1.0 of Chapter 1 it follows that

$$
\|\mu * f\|_{M(Y)} \leq\|\mu\|_{M(Y)}\|f\|_{\nu} .
$$

To show from (4.23) that $\mu * f \in L^{1}(Y)$, it is sufficient to observe that by Proposition 1.0 above, $L^{1}$ is a closed subfunctor of $M$, and since $f \in L^{1}, \mu * f$ may clearly be obtained as the limit in $M(Y)$ of a sequence of integrable functions. Hence

$$
\|\mu * f\|_{L^{\prime}(Y)} \leq\|\mu\|_{M(Y)}\|f\|_{L^{\prime}}
$$

Now recall that by Definition $1.7,\left[L^{\infty}, X\right]$. To obtain a homomorphism $\hat{\mu} \in \operatorname{Hom}_{\otimes}\left[L^{\infty}, L^{\infty}\right](Y)$, define for every $\lambda \in L^{\infty}(X)$ and $X \in \mathcal{B}$

$$
\hat{\mu}(\lambda)=\lambda * \mu(\varphi)=\left(\lambda \otimes 1_{Y}\right) \circ J_{Y}(\varphi * \mu) \quad \forall \varphi \in L^{1}
$$

where $J_{Y}: L^{1}(Y) \rightarrow L^{1} \otimes Y$ is the isomorphism of Theorem 1.3, Chapter 1.

By (4.24), $\varphi * \mu$ defines a homomorphism from $L^{1}$ to $L^{1}(Y)$. By Grothendieck's theorem, Theorem 1.3, $J_{Y}: L^{1}(Y) \cong L^{1} \otimes Y$. Therefore $\left(\lambda \otimes 1_{Y}\right) \circ J_{Y}$ defines a continuous linear mapping from $L^{\prime}(Y)$ to $X^{\prime} \otimes Y$. Since the $Y$-valued measure $\mu$ commutes with any complex-valued measure $\sigma \in M$, it follows that the mapping $\hat{\mu}: L^{\infty}(X) \rightarrow L^{\infty}(X \otimes Y)$ defined by (4.25) is a module homomorphism.

To show that $\gamma$ is an onto it will be enough to show that the operator $\hat{\mu}$ defined by (4.25) yields the measure $\mu=\gamma(\hat{\mu})$ under the homomorphism $\gamma$.

Since $\gamma=i_{y} \circ \delta_{0}^{\gamma} \circ \sigma_{y}$, where $\sigma$ restricts $h \in \operatorname{Hom}_{\circledast}\left[L^{\infty}, L^{\infty}\right]$ to the functor $C$, it will be sufficient to show that the operator (4.25) is of the form (4.20) for every $f \in C(X)$. By Theorem 1.0, $f \in C(X)$ defines a compact linear operator $\lambda_{f}: L^{1} \rightarrow X$ by $\lambda_{f}(\varphi)=\int f(t) \varphi(t) d t$ for all $\varphi \in L^{1}$. Let $\varphi_{y}=\varphi \cdot y \in L^{1}(Y)$, where $\varphi \in L^{1}$ and $y \in Y$. Then $\left(\lambda_{f} \otimes 1_{Y}\right) \circ J_{Y}(\varphi \cdot y)=y \otimes \int f d t=\int f \otimes \varphi_{y} d t$. Since functions of the form $\sum^{N} y_{i} \varphi_{i}$ are dense in $L^{1}(Y)$ and $\left(\lambda_{f} \otimes 1_{Y}\right) \circ J_{Y}$ defines a continuous linear operator on $L^{1}(Y)$ by the isomorphism $J_{Y}$, it follows that $\left(\lambda_{f} \otimes 1_{Y}\right) \circ J_{Y}(g)=\int f \otimes g d t \in X \otimes Y$ for every $f \in C(X)$ and $g \in L^{1}(Y)$.

By (4.25), it follows that

$$
\lambda_{f} * \mu(\varphi)=\int f(t) \otimes(\mu * \varphi)(t) d t \in X \otimes Y .
$$

Since (4.26) is the composition of a linear operator with a compact linear operator, $\lambda_{f} * \mu$ must be a compact operator from $L^{1}$ to $X \otimes Y$. Hence by Theorem 1.2 there exists a continuous function $k \in C(X \otimes Y)$ such that 


$$
\hat{\mu}\left(\lambda_{f}\right)(\varphi)=\lambda_{f} * \mu(\varphi)=\int k(t) \varphi(t) d t
$$

for every $\varphi \in L$, and $k$ may therefore be isomorphically and canonically identified with $\lambda_{f} * \mu$.

Since $\lambda_{f} \in C(X), \sigma_{Y}(\hat{\mu})\left(\lambda_{f}\right)=\hat{\mu}\left(\lambda_{f}\right)$. Therefore by (4.25) and (4.27) above,

$$
\delta_{0}^{Y} \circ \sigma_{Y}(\hat{\mu})\left(\lambda_{f}\right)=\delta_{0}^{Y}(\hat{\mu})\left(\lambda_{f}\right)=k(0)
$$

where $\delta_{0}^{Y} \circ \sigma_{Y}(\hat{\mu}) \in D C(Y)$.

Let $\varphi_{k}$ be an approximate identity in $L^{\prime}$. Since $k$ is continuous,

$$
\begin{aligned}
k(0) & =\lim \int k(t) \varphi_{k}(t) d t=\lim \int f(t) \otimes\left(\mu * \varphi_{k}\right) d t \\
& =\lim \int\left(\int f(t) \varphi_{k}(t-s) d t\right) \otimes d \mu_{s}=\int f(s) \otimes d \mu_{s}
\end{aligned}
$$

where the limits in (4.28) exist by the continuity of $k$ and $f$.

Therefore $\delta_{0}^{Y} \circ \sigma_{Y}(\hat{\mu})\left(\lambda_{f}\right)=\int f(s) \otimes d \mu_{s}$ and hence since $i_{Y}$ is an isomorphism, $i_{Y}(\hat{\mu})=\mu$ and the theorem is proved.

Using Theorem 2.3 above on the properties of the mappings $\Lambda$ and $\tau$, it is now possible to obtain a corollary for mappings in the category $\mathcal{B}_{0}$.

Corollary 4.4.1. Let $T^{n}$ be a toroidal group, $\mathcal{B}_{0}$ the category of harmonically invariant Banach spaces of distributions on $T^{n}$, and $\mathcal{B}$ the category of Banach spaces. Let $L^{\infty}$ and $C$ be the $L^{\infty}$ and $C$ functors acting on $\mathcal{B}_{0}$. Then there exist monomorphic and continuous embeddings

$$
M \subseteq \operatorname{Hom}_{\otimes}[\Lambda C, \Lambda C], \quad M \subseteq \operatorname{Hom}_{\otimes}\left[\Lambda L^{\infty}, \Lambda L^{\infty}\right],
$$

where the functors in (4.29) act on the category $\mathcal{B}$.

Proof. Corollary 4.4.1 follows immediately from part (3) of Theorem 2.3. Let $Y \in \mathcal{B}$ and $\mu \in M(Y)$. Then as in previous theorems $\Lambda^{*}(\mu) * \lambda=\Lambda \mu * \tau \lambda$ $=\mu * \lambda$; hence $\mu * \tau \lambda=\tau(\mu * \lambda)$ and the theorem follows by the isometry $\tau: \Lambda L^{\infty}(X) \rightarrow \Delta L^{\infty}(X)$ which holds for every $X \in \mathcal{B}_{0}$.

By Theorem 2.5, for every $X \in \mathcal{B}_{0}, \Lambda C(X) \subseteq X \subseteq \Lambda L^{\infty}(X)$. For the category $\mathcal{B}_{0}$ it is possible to prove a theorem on complex-valued multipliers for these spaces.

Corollary 4.4.2. Let $T^{n}$ be a toroidal group and $X$ a Banach space $X \in \mathcal{B}_{0}$. Then:

(1) If $M$ is the ring of complex-valued measures on $T^{n}$, then

$$
M \subseteq[\Lambda C(X), \Lambda C(X)], \quad M \subseteq\left[\Lambda L^{\infty}(X), \Lambda L^{\infty}(X)\right] .
$$

(2) If $L^{1}$ is the ring of integrable functions on $T^{n}$, then $L^{1} \subseteq[X, X]$. 
Proof. Part (1) follows directly from Corollary 4.4.1. To show part (2), observe that since $L^{\prime} \subseteq M$, it follows by Corollary 4.4.1 that $\Lambda^{*}$ defines a monomorphic embedding $\Lambda^{*}: L^{1} \rightarrow\left[\Lambda L^{\infty}(X), \Lambda L^{\infty}(X)\right]$. Therefore by Theorem $2.5, \Lambda^{*}: L^{1}$ $\rightarrow\left[X, \Lambda L^{\infty}(X)\right]$. It remains therefore to prove that for every $X \in \mathcal{B}_{0}, h \in L^{1}$, and $\lambda \in X, h * \lambda \in X$.

Since $h \in L^{1}, h$ is the limit in $L^{1}$ of a sequence of trigonometric polynomials $P_{N}=\Sigma^{N} e_{m} e_{m}$. By the harmonic invariance of $X c_{m} e_{m} * \lambda=c_{n} \lambda(m) e_{m} \in X$. Therefore for every $P_{N}$ and $\lambda \in X, P_{N} * \lambda \in X$. Since the mapping $\Lambda^{*}: L^{1}$ $\rightarrow\left[X, \Lambda L^{\infty}(X)\right]$ is continuous and $X$ is a closed subspace of $\Lambda L^{\infty}(X)$ for every $X \in \mathcal{B}_{0}$, it follows that $P_{N} * \lambda \rightarrow h * \lambda$ in the norm of $X$, and hence $h * \lambda \in X$.

Part (2) of Corollary 4.4 .2 implies in particular that every Banach space $X \in \mathcal{B}_{0}$ will also be contained in $\mathcal{-} \mu_{0}$; thus as classes of Banach spaces $\mathcal{B}_{0} \subseteq M_{0}$, and hence (again as classes of Banach spaces) $\mathcal{B}_{0}=\mathcal{d}_{0}^{\prime}$. Corollary 4.4.2 also provides a general setting in the category $\mathcal{B}_{0}$ for a theorem proved by Katznelson in [15] to the effect that the ring $M$ of complex-valued measures was a class of multipliers on any homogeneous Banach space $X$ (see the remarks following Corollary 3.1.1). Corollary 4.4 .2 seems to me of special interest in that very many spaces employed in analysis, such as continuity spaces or spaces $M_{A}$, $L_{A}, K_{A}$ with $A$ of rapid growth, are harmonically invariant though not homogeneous; it therefore seemed to me useful to treat of "universal multipliers" in the widest setting possible.

Corollary 4.4.1 states that the functor $M$ defines an important subclass of the tensor multipliers $\operatorname{Hom}_{\otimes}\left[\Lambda L^{\infty}, \Lambda L^{\infty}\right]$ and $\operatorname{Hom}_{\otimes}[\Lambda C, \Lambda C]$. By closely similar methods to those of Corollary 4.4 .2 it is possible to prove a weaker theorem characterizing a similar class for the tensor multipliers $\mathrm{Hom}_{\otimes}[I, I]$ where $I$ is the identity functor on the category $c M_{0}^{\prime}$.

Corollary 4.4.3. Let $T^{n}$ be a toroidal group, $c M_{0}^{\prime}$ the category of normal modules of distributions on $T^{n}$, in $\mathcal{B}_{0}$, and $\mathcal{B}$ the category of Banach spaces. Let $I$ be the identity functor on $\mathcal{M}_{0}^{\prime}$ and $L^{1}$ the $L^{\prime}$ functor on $\mathcal{B}$. Then there exists a continuous monomorphic embedding

$$
L^{1} \subseteq \operatorname{Hom}_{\otimes}[I, I]
$$

where the functors in (4.30) act on the category $\mathcal{B}$.

Proof. The proof of Corollary 4.4 .2 may be imitated for vector-valued multipliers $h \in L^{1}(Y)$ and tensor convolution; note that since $h \in L^{1}(Y), h$ is again the limit in $L^{1}(Y)$ of a sequence of trigonometric polynomials $P_{N}$ $=\sum^{N} y_{m} e_{m}$ where $y_{m} \in Y$. It then follows in the same way as above that $h * \lambda \in X \otimes Y$ for every $\lambda \in X$.

Corollaries 4.4.2 and 4.4.3 have direct application to the case of integrals with a parameter. An example, which appears in the study of parabolic equations, is the Poisson-Weierstrass integral 


$$
H f(x, t)=\int_{\Omega} \frac{f(y)}{t^{n / 2}} \exp \left[\frac{-(x-y)^{2}}{4 t}\right] d y=\int_{\Omega} h(t, x-y) f(y) d y
$$

over an $n$-dimensional bounded region $\Omega$, which may be considered as a subset of the $n$-dimensional toroid. The kernel $h$ is contained in $L^{1}\left(L^{1}\right)=L^{1} \otimes L^{1}$. It therefore follows from Corollary 4.4.3 that for every $X \in B_{0}, H: X \rightarrow X \otimes L$. A discussion of operators of the type (4.31) and their role in parabolic partial differential equations appears in Pogorzelski [29].

By using the properties of $\otimes$ as a universal bilinear mapping other results of interest for applications may be obtained.

Corollary 4.4.4. Let $T^{n}$ be a toroidal group, $X$ a Banach module, $X \in \triangle M_{00}$, and $[X, X]$ the algebra of convolution multipliers on $X$. Suppose that $\Lambda L^{\infty}(X)=X$ or $\Lambda C(X)=X$. Then for every $H, K \in M([X, X])$, the convolution $\Lambda^{*}(H * K)$ defines an element of $[X, X]$.

Proof. I shall first show that $M([X, X])$ forms an algebra under convolution.

Let $H, K \in M([X, X])$. Then by Definition 1.21 above it follows that $H * K$ $\in M([X, X] \otimes[X, X])$ where $H \otimes K$ is the tensor convolution of $H$ and $K$.

Since $\otimes$ is a universal bilinear map, the mapping $\langle,\rangle_{[X, X]}:[X, X] \otimes[X$, $\rightarrow[X, X]$ defined by $\langle h, k\rangle_{[X, X]}=h k$, the operator composition of $h$ and $k$, is a continuous bilinear mapping. Since $H \otimes K \in M([X, X] \otimes[X, X])$, it follows that $\langle H * K\rangle=H *_{0} K \in M([X, X])$ and hence that $M([X, X])$ forms an algebra under the operation *0.

By Corollary 4.4.3, $\Lambda(H) \tau \in\left[\Lambda L^{\infty}(X), \Lambda L^{\infty}([X, X] \otimes X)\right]$ for every $H$ $\in M([X, X])$. Let $f \in \Lambda L^{\infty}(X)$ and $H \in M([X, X])$. Then

$$
\begin{aligned}
\Lambda(H) \tau f & =\frac{1}{(2 \pi)^{n}} \int L^{\infty}\left(1_{\{X, X]} \otimes \delta_{-p}\right)\left[\int H(s-t) \otimes f_{t} d t\right]_{p} d p \\
& =\frac{1}{(2 \pi)^{n}} \iint H(s+p-t) \otimes f_{t-p} d t d p=\int H(s-t) \otimes f_{t} d t .
\end{aligned}
$$

Hence $\Lambda H \tau \in \Lambda L^{\infty}(X) \rightarrow \Lambda L^{\infty}([X, X] \otimes X)$ and $H \tau=\tau H$. Since $\otimes$ is universal, the bilinear mapping $\langle,\rangle_{X}:[X, X] \otimes X \rightarrow X$ defined by $\langle h, f\rangle_{X}=h f$ is continuous. Therefore

$$
\left\langle\int H(s-t) \otimes f_{t} d t\right\rangle=\int H(s-t) f_{t} d t=\Lambda H \tau f
$$

defines a continuous module homomorphism $\Lambda H \tau: \Lambda L^{\infty}(X) \rightarrow \Lambda L^{\infty}(X)$. Since $X \in M_{\infty}$, it follows that $\Lambda H \tau: X \rightarrow X$ and Corollary 4.4.4 is proved.

The interest of Corollary 4.4.4 is in showing that the class $[X, X]$ is closed under the formation of the measure algebra $M([X, X])$. A less general result for $H \in L^{\prime}\left(L^{\prime}\right)$ is easy to show. Let $H \in L^{\prime}\left(L^{\prime}\right)$. Then it is easy to see that 


$$
\begin{aligned}
\Delta H \tau f & =\frac{1}{(2 \pi)^{n}} \iint H(s-t, x-y) f(y+t) d y d t \\
& =\frac{1}{(2 \pi)^{n}} \int\left[\int H(s-t, x+t-u) d t\right] f(u) d u \\
& =\int\left[\frac{1}{(2 \pi)^{n}} \int H(v, x+s-u-v) d v\right] f(u) d u \\
& =\int\left[\int H(v, x-u-v) d v\right] f(u) d u .
\end{aligned}
$$

Thus $\Delta H \tau f=H \tau f$ and $\Lambda H \tau: X \rightarrow X$ for any $X \in M_{00}$.

A third corollary deals with a type of operator very common in the theory of integral and differential operators.

Corollary 4.4.5. Let $T^{n}$ be a toroidal group and $X$ a Banach function module over $M_{0}$ and $L^{\infty}$. Let $h \in L^{1}\left(L^{\infty}\right)$ and define an operator

$$
H f(x)=\int h(x, x-y) f(y) d y
$$

for every $f \in X$. Then $H: \Lambda L^{\infty}(X) \rightarrow \Lambda L^{\infty}(X)$.

Proof. Since $h \in L^{\prime}\left(L^{\infty}\right) \subseteq M\left(L^{\infty}\right)$, Theorem 4.4 implies that $h \in$ $\operatorname{Hom}_{\circledast}\left[L^{\infty}, L^{\infty}\right]\left(L^{\infty}\right)$. Since $h \in L^{1}\left(L^{\infty}\right),\left\|h_{s}-h\right\|_{L^{\prime}\left(L^{\infty}\right)} \rightarrow 0$ as $s \rightarrow 0$ and hence $h \in \operatorname{Hom}_{\odot}\left[L^{\infty}, C\right]\left(L^{\infty}\right)$. Therefore if $H_{0}$ is the operator defined by

$$
H_{0} f(x)=\int h(x-y) \otimes f(y) d y \quad \text { for } h \in L^{1}\left(L^{\infty}\right)
$$

then $H_{0}: L^{\infty}(X) \rightarrow C\left(X \otimes L^{\infty}\right)$.

For every $f \in C\left(X \otimes L^{\infty}\right)$ define a map $e: C\left(X \otimes L^{\infty}\right) \rightarrow L^{\infty}\left(X \otimes L^{\infty}\right)$ by

$$
e(f)(x)=\left(1_{X} \otimes \delta_{x}\right)(f)(x)=e_{x} \circ C\left(1_{X} \otimes \delta_{x}\right)(f)
$$

where $\delta_{x}$ is the operator of translation by $x$ applied to $L^{\infty}$, and $e_{x}$ is the operator of evaluation at $x$, applied to the space of functions $f \in C\left(X \otimes L^{\infty}\right)$.

An example will illustrate the properties of the mapping $e$ defined by (4.34): let $X$ be a Banach space of functions. For every $f \in C\left(X \otimes L^{\infty}\right), f$ is a continuous function from $T^{n}$ to $X \otimes L^{\infty}$; thus $f(p, q)(t)=x(p) \otimes \varphi(q)(t)$ where $t \in T^{n}$ and $p, q$ are the variables for $x \in X$ and $\varphi \in L^{\infty}$ respectively. Then $\delta_{x}$ operates on $L^{\infty}$ and $e_{x}$ evaluates $f$ at $x$; thus for every $x$

$$
e(f)(x)=x(p) \otimes \varphi(q+x)(x)
$$

Finally let $m: X \otimes L^{\infty} \rightarrow X$ be the bilinear operator defined by module multiplication of $X$ and $L^{\infty}$. Since $m: X \otimes L^{\infty} \rightarrow X$ is continuous the mapping $L^{\infty}(m): L^{\infty}\left(X \otimes L^{\infty}\right) \rightarrow L^{\infty}(X)$ is continuous.

The action of $m$ on $e(f)$ in (4.34) results in $\left[L^{\infty}(m) \circ e(f)\right](x)=m \circ e(f)(x)$ $=x(p) \varphi(p+x)(x)$ for every $x \in T^{n}$. 
Now let $H_{0}^{\prime}: L^{\infty}(X) \rightarrow L^{\infty}(X)$ be defined by

$$
H_{0}^{\prime} f=L^{\infty}(m) \circ e \circ H_{0}(f)
$$

where $H_{0}$ is defined by (4.33). Clearly $H_{0}^{\prime}$ is continuous as the composition of continuous operators. Furthermore

$$
\begin{aligned}
\Delta H_{0}^{\prime} \tau f & =\frac{1}{(2 \pi)^{n}} \int \delta_{-s}^{X}\left[L^{\infty}(m) \int \delta_{x}^{L^{\infty}} h(x-y) \otimes \tau f(y) d y\right]_{s} d s \\
& =\frac{1}{(2 \pi)^{n}} \int \delta_{-s}^{X}\left[\int \delta_{x}^{L^{\infty}} h(x-y) \tau f(y) d y\right]_{s} d s \\
& =\frac{1}{(2 \pi)^{n}} \int \delta_{-s}^{X} \int \delta_{x+s}^{L^{\infty}} h(x+s-y) \tau f(y) d y d s \\
& =\frac{1}{(2 \pi)^{n}} \iint \delta_{x}^{L^{\infty}} h(x+s-y) \delta_{-s}^{X} \tau f(y) d y d s \\
& =\frac{1}{(2 \pi)^{n}} \iint \delta_{x}^{L^{\infty}} h(x+s-y) \tau f(y-s) d y d s \\
& =\int \delta_{x}^{L^{\infty}} h(x-y) \tau f(y) d y=H_{0}^{\prime} \tau f .
\end{aligned}
$$

Since $\Delta: L^{\infty}(X) \rightarrow \Delta L^{\infty}(X)$ is a projection, it follows that $H_{0}^{\prime}$ defines an operator $H_{0}: \Delta L^{\infty}(X) \rightarrow \Delta L^{\infty}(X)$. Hence $\Lambda H_{0}^{\prime} \tau: \Lambda L^{\infty}(X) \rightarrow \Lambda L^{\infty}(X)$. It is clear by inspection that $\Lambda H_{0}^{\prime} \tau=H$.

Remark 1. An important class of operators to which Corollary 4.4.5 applies is the class of weakly singular integral operators. Let $X$ be a Banach function module over $M$ and $L^{\infty}$ on a toroidal group $T^{n}$. An integral operator $H_{0}: X \rightarrow X$ is weakly singular if it is of the form

$$
H_{0} f=\int_{\Omega} \frac{c(x, y)}{(x-y)^{\alpha}} f(y) d y
$$

for every $f \in X$, where $\alpha<n$ and $c$ is a continuous function $c: T^{n} \times T^{n} \rightarrow \mathbf{C}$, $c \in C_{T^{n} \times T^{n}}$.

To apply Corollary 4.4 .5 to operators of type (4.35), let $S: T^{n} \times T^{n} \rightarrow T^{n} \times T^{n}$ be the transformation matrix

$$
S=\left[\begin{array}{cc}
I_{n} & I_{n} \\
0 & -I_{n}
\end{array}\right]
$$

where $I_{n}$ is the identity matrix on $T^{n}$. Clearly $S^{2}=I_{2 n}$ and thus $S$ is its own inverse. Define a function $c_{0}=c \circ S$. Then clearly $c_{0}(x, x-y)=c(x, y)$; therefore

$$
H_{0} f=\int \frac{c(x, y)}{(x-y)^{\alpha}} f(y) d y=\int \frac{c_{0}(x, x-y)}{(x-y)^{\alpha}} f(y) d y
$$

and the operator $H_{0}$ is of the form (4.32) so that Corollary 4.4 .5 may be applied. 
Remark 2. It is easy to see that Corollary 4.4 .5 or variants may be extended to deal with some Banach modules of distributions over stronger spaces than $L^{\infty}$ : as for instance, Banach modules over $C$ or $C^{k}$.

In [18] from stronger assumptions Lorentz obtains a stronger result: if $X$ is a Banach function module over $L^{\infty}$, with norm invariant under all measure preserving transformations (not simply translation invariant) then any operator in $L^{\infty}\left(L^{1}\right)$ will act continuously from $X$ to $X$. Corollary 4.4 .5 seems of interest because it shows that simple translation invariance is sufficient to obtain useful results.

In the theory of differential and integral equations several authors [6], [22], [28] have studied the problem of characterizing those pairs of spaces $(X, Y)$ such that a given operator $H$ maps $X$ to $Y$. This question is termed the question of admissibility. The results of Theorem 4.4 and Corollaries 4.4 .2 and 4.4 .5 imply that the pair $(X, X)$ is admissible for equations of the type (4.35), for any space $X \in \mathcal{B}_{0}$ that is also a module over $L^{\infty}$.

A second type of continuity functor for which a complete characterization of the tensor multiplier functor is possible is that of the Lipschitz functors $C^{r}$ for $0<r<1$ (and indeed, with a suitable definition, for $r$ real). Function classes of the type $C^{r}$ have been termed classes of "smooth" functions; A. Zygmund has studied such functions in a long paper [43] and somewhat later gave a characterization of the multipliers for the classes $C^{r}$ in [44]. However Zygmund's original characterization was made for the case of the group $T^{1}$ only. In later work M. Taibleson [41], in a general study of functions of Lipschitz types, developed the fundamental machinery for the study of smooth functions on a general toroidal group $T^{n}$. The characterization of tensor multipliers which I shall give below in Theorem 4.5 for general toroidal groups $T^{n}$ extends the basic ideas of the papers [41] and [44] to the context of vector-valued functions.

I shall now present the basic definitions necessary for the formulation and proof of Theorem 4.5.

Definition 4.2. Let $T^{n}$ be a toroidal group and $B$ the category of Banach spaces; let $M$ be the measure functor $M: B \rightarrow \mathcal{B}$ and $L^{\infty}$ the $L^{\infty}$ functor $L^{\infty}: \mathcal{B} \rightarrow \mathcal{C}$. For every real number $r, 0<r<2$, define functors

$$
\begin{aligned}
W M^{\prime}(X) & =\left\{\mu:\left.C \rightarrow X\left|\left\|\mu_{s}+\mu_{-s}-2 \mu\right\| \leq k\right| s\right|^{r}\right\}, \\
W M^{\prime}(\alpha) & =\alpha \circ \mu, \\
\|\mu\|_{W^{\prime}(X)} & =\|\mu\|_{M(X)}+\sup _{s} \frac{\left\|\mu_{s}+\mu_{-s}-2 \mu\right\|}{|s|^{r}} M(X) ; \\
M^{r}(X) & =\left\{\mu:\left.C \rightarrow X\left|\left\|\mu_{s}-\mu\right\| \leq K\right| s\right|^{r}\right\}, \\
M^{\prime}(\alpha) & =\alpha \circ \mu, \\
\|\mu\|_{M^{\prime}(X)} & =\|\mu\|_{M}+\sup _{s} \frac{\left\|\mu_{s}-\mu\right\|}{|s|^{r}} M(X) ;
\end{aligned}
$$




$$
\begin{aligned}
W C^{\prime}(X) & =\left\{\lambda:\left.L^{1} \rightarrow X\left|\left\|\lambda_{s}+\lambda_{-s}-2 \lambda\right\| \leq k\right| s\right|^{r}\right\}, \\
W C^{r}(\alpha) & =\alpha \circ \lambda, \\
\|\lambda\|_{W C^{\prime}(X)} & =\|\lambda\|_{L^{\infty}(X)}+\sup _{s} \frac{\left\|\lambda_{s}+\lambda_{-s}-2 \lambda\right\|}{|s|^{r}} L^{\infty}(X) ; \\
C^{\prime}(X) & =\left\{\lambda:\left.L^{1} \rightarrow X\left|\left\|\lambda_{s}-\lambda\right\| \leq K\right| s\right|^{r}\right\}, \\
C^{\prime}(\alpha) & =\alpha \circ \lambda, \\
\|\lambda\|_{C^{\prime}(X)} & =\|\lambda\|_{L^{\infty}(X)}+\sup _{s} \frac{\left\|\lambda_{s}-\lambda\right\|}{|s|^{r}} L^{\infty}(X) .
\end{aligned}
$$

Remarks. The following observations may be made. It is easy to see that the functors $M^{\prime}$ and $C^{\prime}$ are trivial for $r>1$ : it is also an easy consequence of Theorem 1.1 that a measure $\mu \in W M^{r}(X)$ or $\mu \in M^{r}(X)$ for $r>0$ must correspond to a function in $L^{1}(X)$; and similarly the linear functional $\lambda$ $\in W C^{r}(X)$ or $\lambda \in C^{\prime}(X)$ must correspond to an element of $C(X)$. The functor (4.38) is the Lipschitz functor which has also been defined above.

In the technical machinery of their proofs both Zygmund and Taibleson use properties of Bessel transforms and Poisson integrals in an essential way; since I shall use analogous arguments it will first be necessary to develop some of the properties of Bessel transforms and Poisson integrals of vector-valued functions.

If $T^{n}$ is a toroidal group, I shall let $T_{+}^{n+1}$ denote the space $T^{n} \times R^{+}$where $R^{+}=\{y \in R \mid y>0\}$. Every function on $T^{n}$ clearly has a periodic extension to $E^{n}$; I shall say that a function on $T_{+}^{n+1}$ or $T^{n}$ is continuous, differentiable, or harmonic if its periodic extension to $E^{n}$ is continuous, differentiable, or harmonic respectively.

A vector-valued harmonic function in $T_{+}^{n+1}$ is a function which satisfies $\Delta u=0$ in the region $T_{+}^{n+1}$, where $\Delta u=\sum_{i=0}^{n} \partial^{2} u / \partial x_{i}^{2}$ and $x_{0}=y$.

The following proposition will be useful.

Proposition 4.2. Let $T^{n}$ be a toroidal group, $\mathcal{B}$ the category of Banach spaces, and $M$ the measure functor on $\mathcal{B}$ over $T^{n}$. Let $h \in L^{1}$ on $T^{n}$. Then for every $X \in \mathcal{B}$, $\mu \in M(X)$ and $\varphi \in C$, define $h_{X}: M(X) \rightarrow M(X)$ by

$$
h_{X}(\mu)(\varphi)=\mu(h * \varphi)
$$

then $h$ defines a natural mapping $h: M \rightarrow M$ by the definition (4.40).

Proof. It is clear from (4.39) that $h_{X}: M(X) \rightarrow M^{*}(X)$. To show that $h_{X}(\mu)$ $\in M(X)$ if $\mu \in M(X)$, let $\left\{\varphi_{i}\right\}_{i=1}^{N}$ be a finite set of continuous functions. Then

$$
\begin{aligned}
\sum^{N}\left\|h_{X}(\mu)\left(\varphi_{i}\right)\right\| & =\sum^{N}\left\|\mu\left(h * \varphi_{i}\right)\right\| \leq\|\mu \mu\| \sup \left\|\sum^{N} \varepsilon_{i} h * \varphi_{i}\right\| \\
& \leq\|\| \mu\|\|\|h\|_{h} \sup \left\|\sum^{N} \varepsilon_{i} \varphi_{i}\right\|
\end{aligned}
$$


where the suprema in (4.41) are taken over all sets $\left\{\varepsilon_{i}\right\}_{i=1}^{N}$ such that $\left|\varepsilon_{i}\right| \leq 1$ for every $i$. It follows from $(4.41)$ and the definition of the norm in $M(X)$ that $\left|\left\|h_{X}(\mu)\right\|\right| \leq\|h\|_{1} \mid\|\mu\|$ and hence $h_{X}(\mu) \in M(X)$.

To show that $h$ is natural, let $X, Y \in \mathcal{B}$ and $\alpha \in[X, Y]$. Then

$$
M(\alpha) h_{X}(\mu)(\varphi)=\alpha\left[h_{X}(\mu)(\varphi)\right]=\alpha[\mu(h * \varphi)]=h_{Y} M(\alpha) \mu(\varphi)
$$

which is merely the statement that the diagram

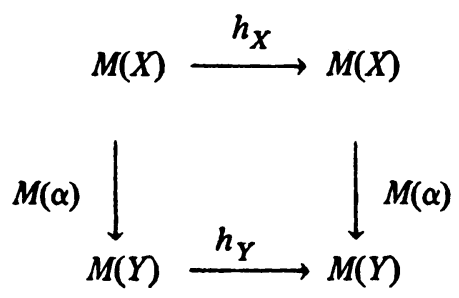

commutes.

Definition 4.3. The Poisson kernel for $T_{+}^{n+1}$ is defined as

$$
(t, y)=\sum_{m} P(t+m, y)=\sum_{m} e^{2 \pi y|m|} e^{2 \pi i\langle m, t\rangle}
$$

where $P(t, y)=c_{n}^{-1} y /\left(|t|^{2}+y\right)^{(n+1) / 2}, m=\left(m_{1}, m_{2}, \ldots, m_{n}\right)$ where all $m_{i}$ are integers, $c_{n}=\Pi(n+1) / \Gamma((n+1) / 2)$. Let $\mathcal{B}$ be the category of Banach spaces and $M$ the measure functor on $T^{n}$. Then for every $y>0$ the Poisson kernel defines a natural transformation $P_{y}^{*}: M \rightarrow M$ by

$$
y, x^{*} \mu(f)=\mu(, * f)
$$

for every $\mu \in M(X), X \in \mathcal{B}$, and $f \in C$.

Since $P_{y}^{*} \in L^{1}$ for every $y>0$ it follows by Proposition 4.2 that $P_{y}^{*}$ defines a natural transformation $P_{y}^{*}: M \rightarrow M$ and in particular is a continuous module homomorphism from $M(X)$ to $M(X)$ for every $X \in \mathcal{B}$. In fact from (4.43) it is clear that for $y>0,(t, y)$ is infinitely differentiable in $t$, and hence it follows again by Theorem 1.1 that for every $X \in \mathcal{B}, y>0$, and $\mu \in M(X), P_{y}^{*} * \mu$ may be identified with an $X$-valued infinitely differentiable function. I shall denote this function by $\lambda(t, y)$ for $y>0$.

The following proposition states some salient properties of the kernels $(t, y)$. It is well known.

Proposition 4.3. Let $P^{*}(t, y)$ be the Poisson kernel defined by (4.42). Then

(1) $P^{*}(t, y) \geq 0$,

(2) $\int_{T^{n}} P^{*}(t, y) d t=1 \forall y>0$,

(3) $\int_{|t| \leq s} P(t, y) d t \rightarrow 0$ as $y \rightarrow 0 \forall s>0$.

It will also be necessary to give a definition of the Bessel potentials.

Definition 4.4. Let $r$ be a real number $r>0$. Define a kernel $J^{r}(t)$ by 


$$
J^{r}(t)=\sum_{m}\left(1+|m|^{2}\right)^{-r / 2} \exp 2 \pi i\langle m, t\rangle .
$$

Let $\mathcal{B}$ be the category of Banach spaces and $M$ the measure functor on $T^{n}$. Then for every $X \in \mathcal{B}$ and $\mu \in M(X)$, the Bessel transform of $\mu \in M(X)$ is defined by

$$
\mu * J_{X}^{r}(f)=\mu\left(J^{r} * f\right) \quad \text { for every } f \in C .
$$

The kernel $J^{r}$ for $r>0$ satisfies

$$
\begin{aligned}
J^{r}(t) & \geq 0, & & \int J^{r}(t) d t=1, \\
J^{n} \times J^{r} & =J^{n+r_{2}} & & \text { for } r_{1}, r_{2}>0, \\
J^{r} & \in W M^{r} & & \text { for } 0<r<2, \\
J^{r} & \in M^{r} & & \text { for } 0<r<1 .
\end{aligned}
$$

Since $J^{r} \in L^{1}$ for every $r>0$, it follows by Proposition 4.2 that the mappings $J_{X}^{r}$ defined in (4.44) define a natural mapping from the functor $M$ to $M$ by convolution. I shall denote this mapping by $J^{r}: M \rightarrow M$ and write $J^{r} \mu=J^{r} * \mu$.

Using the Bessel potential it is now possible to define the functor $M J$.

Definition 4.5. Let $T^{n}$ be a toroidal group and $\mathcal{B}$ the category of Banach spaces. Let $M J: \mathcal{B} \rightarrow \mathcal{C}$ be defined by

$$
\begin{aligned}
& M J(X)=\left\{\mu: C^{\infty} \rightarrow X \mid \mu * J^{1} \in W M^{1}\right\} \\
& \quad \text { where } J^{1} \text { is the Bessel potential (4.44) for } r=1, \\
& M J(\alpha)=\alpha \circ \mu, \\
& \|\mu\|_{M J}=\left\|\mu * J^{1}\right\|_{W M^{1}} .
\end{aligned}
$$

It is now possible to state and prove a theorem characterizing the tensor multiplier functor $\mathrm{Hom}_{\otimes}\left[C^{r}, C^{r}\right]$.

Theorem 4.5. Let $T^{n}$ be a toroidal group and $\mathcal{B}$ the category of Banach spaces. Let $C^{r}: B \rightarrow \propto M$ be the Lipschitz functor for $0<r<1$. Then

$$
M J \cong \mathrm{Hom}_{\otimes}\left[C^{r}, C^{r}\right]
$$

Proof. I shall first prove a series of lemmas giving the main properties of the functors $C^{r}, W C^{r}, M^{r}, W M^{r}$. From these lemmas the theorem will follow easily.

Lemma 4.5.1. Suppose that $\lambda(t, y)$ and $\mu(t, y)$ are harmonic Banach space-valued functions in $T_{+}^{n+1}$ and for every $y_{0}>0, \lambda_{y}(t)=\lambda(t, y)$ and $\mu_{y}(t)=\mu(t, y)$ are uniformly bounded for $y \geq y_{0}$. Let $\lambda_{y}: T^{n} \rightarrow X$ and $\mu_{y}: T^{n} \rightarrow Y$. Then:

(1) $\lambda_{y_{1}} * \mu_{y_{2}}=\lambda_{y_{1}+y_{3}} * \mu_{y_{2}-y_{3}}$ for all $y_{1}, y_{2}, y_{3}$ such that $y_{1}+y_{3}>0, y_{2}-y_{3}>0$, $y_{1}, y_{2}>0$. 
(2) $\gamma_{y}=\lambda_{y_{1}} * \mu_{y_{2}}\left(y_{1}, y_{2}>0, y_{1}+y_{2}=y\right)$ is well defined for all $y>0$.

(3) $\gamma_{y}^{\prime}=\lambda_{y_{1}}^{\prime} * \mu_{y_{2}}=\lambda_{y_{1}} * \mu_{y_{2}}^{\prime}$ for $y=y_{1}+y_{2}$ where $\gamma^{\prime}, \lambda^{\prime}, \mu^{\prime}$ denote the derivatives with respect to $y$.

(4) $\gamma_{y}(t)=\gamma(t, y)$ is harmonic in $T_{+}^{n+1}$ and $\gamma_{y}: T^{n} \rightarrow X \otimes Y$ for every $y>0$.

* in (1), (2), (3) denotes tensor convolution.

Proof. Let $Z$ be a Banach space and let $\gamma(t, y)=\gamma_{y}(t)$ be a harmonic function $\gamma_{y}: T^{n} \rightarrow Z$ uniformly bounded for all $y \geq y_{0}>0$ and all $y_{0}$. Observe that

(a) $\gamma_{y_{1}+y_{2}}=\gamma_{y_{1}} * P_{y_{2}}$,

(b) $\quad \gamma_{y_{1}+y_{2}}^{\prime}=\gamma_{y_{1}}^{\prime} * P_{y_{2}}=\gamma_{y_{1}} * P_{y_{2}}^{\prime}$.

To see (a), for instance, recall that since (4.45) holds for the scalar-valued case [41, 2.12], $\left\langle z^{*}, \gamma_{y_{1}+y_{2}}\right\rangle=\left\langle z^{*}, \gamma_{y_{1}}\right\rangle * P_{y_{2}}=\left\langle z^{*}, \gamma_{y_{1}} * P_{y_{2}}\right\rangle$ for all $z^{*} \in Z^{*}$, and therefore (a) holds. The same type of argument is valid for (b).

Now obtain (1), let $y_{3}>0$ and observe that

$$
\lambda_{y_{1}} * \mu_{y_{2}}=\lambda_{y_{1}} *\left(\mu_{y_{2}-y_{3}} * P_{y_{3}}\right)=\left(\lambda_{y_{1}} * P_{y_{3}}\right) * \mu_{y_{2}-y_{3}}=\lambda_{y_{1}+y_{3}} * \mu_{y_{2}-y_{3}}
$$

by (4.45)(b). A similar argument will show (3). (2) follows immediately from (1); (4) follows immediately from (2).

Lemma 4.5.2. Let $T^{n}$ be a toroidal group and $\mathcal{B}$ the category of Banach spaces.

Define for all $r>0$ and every integer $k \geq r$ and every $X \in \mathcal{B}$,

$$
\begin{aligned}
\|\lambda\|_{C^{k}(X)}= & \sup _{y>0}\left\|y^{k-r} \lambda_{y}^{(k)}\right\|_{L^{\infty}(X)} \\
& \text { where } \lambda_{y}^{(k)} \text { denotes the kth derivative of } \lambda_{y} \text { with respect to } y, \\
\|\mu\|_{M, k(X)}= & \sup _{y>0}\left\|y^{k-r} \mu_{y}^{(k)}\right\|_{M(X)} \quad \text { where } \mu \in M(X) .
\end{aligned}
$$

Then

(1) Let $0<r<2$ and let $\bar{r}$ denote the smallest integer greater than $r$. Then for every $k \geq r$ there exists a constant $A_{k}^{r}>0$ such that

$$
\begin{aligned}
\|\lambda\|_{C k(X)} / A_{k}^{r} & \leq\|\lambda\|_{C_{k}^{j}(X)} \leq A_{k}^{r}\|\lambda\|_{C^{k}(X)}, \\
\|\mu\|_{M^{k}(X)} / A_{k}^{r} & \leq\|\mu\|_{M \bar{r}(X)} \leq A_{k}^{r}\|\mu\|_{M_{r}^{k}(X) .}
\end{aligned}
$$

(2) Let $0<r<2$. Then there exists a constant $B_{r}>0$ such that

$$
\begin{aligned}
& \|\lambda\|_{C^{2}(X)} / B_{r} \leq\|\lambda\|_{W C^{\prime}(X)} \leq B_{r}\|\lambda\|_{C^{2}(X)}, \\
& \|\mu\|_{M^{2}(X)} / B_{r} \leq\|\mu\|_{W M^{\prime}(X)} \leq B_{r}\|\mu\|_{M^{2}(X)} .
\end{aligned}
$$

(3) Let $0<r<1$. Then there exists a constant $C_{r}>0$ such that

$$
\begin{gathered}
\|\lambda\|_{C^{\jmath}(X)} / C_{r} \leq\|\lambda\|_{C^{\prime}(X)} \leq C_{r}\|\lambda\|_{C^{\jmath}(X)}, \\
\|\mu\|_{M^{\prime}(X)} / C_{r} \leq\|\mu\|_{M^{\prime}(X)} \leq C_{r}\|\mu\|_{M^{\prime}(X)}
\end{gathered}
$$


In all cases the norm equivalences are independent of the Banach space $X \in \mathcal{B}$. Therefore $C_{r}^{k}$ and $M_{r}^{k}$ define functors identical to within equivalence of norms to $C^{r}$, $M^{r}, W C^{r}, W M^{r}$ depending on the value of $r>0$.

Proof. The general mode of proof in each case will be the same. By Taibleson [41, Theorem 4, p. 421], all of the equivalences of Lemma 4.5.2 are true for the scalar-valued case $X=C$.

Let $X \in \mathcal{B}$ and let $P_{y}$ be the Poisson kernel (4.42). Suppose that $\lambda \in L^{\infty}(X)$ $=\left[L^{1}, X\right]$ is an element of $C_{r}^{k}(X)$, and let $\lambda^{*}$ be the dual of $\lambda . \lambda^{*}: X^{*} \rightarrow L^{\infty}$. For every $x^{*} \in X^{*}$ and $\varphi \in L^{1}$,

$$
\begin{aligned}
\left\langle\lambda_{y}^{*}\left(x^{*}\right), \varphi\right\rangle & =\left\langle x^{*}, \lambda_{y}(\varphi)\right\rangle=\left\langle x^{*}, \lambda\left(P_{y} * \varphi\right)\right\rangle \\
& =\left\langle\lambda^{*}\left(x^{*}\right), P_{y}^{*} \varphi\right\rangle=\left\langle\lambda^{*}\left(x^{*}\right) * P_{y}, \varphi\right\rangle=\left\langle\lambda^{*}\left(x^{*}\right)_{y}, \varphi\right\rangle .
\end{aligned}
$$

Hence

$$
\begin{aligned}
\left\|\lambda_{y}^{*}(k)\right\|_{\left[x, C^{k}\right]} & =\sup _{x^{*}} \sup _{y>0}\left\|\lambda^{*}\left(x^{*}\right)_{y}\right\|_{b_{0}} y^{k-r}=\sup _{y>0} \sup _{x^{*}}\left\|\lambda^{*}\left(x^{*}\right)_{y}\right\|_{b_{0}} y^{k-r} \\
& =\sup _{y} \sup _{x^{*}} \sup _{\varphi} y^{k-r}\left\langle\lambda^{*}\left(x^{*}\right)_{y}, \varphi\right\rangle=\sup _{y} \sup _{x^{*}} \sup _{\varphi} y^{k-r}\left\langle\lambda_{y}^{*}\left(x^{*}\right), \varphi\right\rangle \\
& =\sup _{y}\left\|\lambda_{y}^{*}\right\|_{x^{*}, L^{\infty}} y^{k-r}=\sup _{y}\left\|\lambda_{y}\right\|_{(v, x)} y^{k-r}=\|\lambda\|_{C k}(x) .
\end{aligned}
$$

To show 1(a) it is therefore sufficient to observe that by the equivalence of the norms of $C_{r}^{k}$ and $C_{r}^{\bar{r}}, C_{r}^{k}(X)$ and $C_{r}^{\bar{r}}(X)$ must be equivalent for all $X$. Since the constants for the equivalence of $C_{r}^{k}$ and $C_{r}^{\bar{r}}$ clearly do not depend on $X \in \mathcal{B}$, the constant $A_{k}^{r}$ is independent of $X$.

Suppose again that $\lambda \in L^{\infty}(X)=\left[L^{1}, X\right]$ is an element of $W C^{r}(X)$. Let $\lambda_{s}$ denote the translation of $\lambda$ by $s$ defined in the natural way; then again for every $x^{*} \in X^{*}$ and $\varphi \in L^{\prime}$,

$$
\begin{aligned}
\left\langle\lambda_{s}^{*}\left(x^{*}\right), \varphi\right\rangle & =\left\langle x^{*}, \lambda_{s}(\varphi)\right\rangle=\left\langle x^{*}, \lambda\left(\varphi_{-s}\right)\right\rangle \\
& =\left\langle\lambda^{*}\left(x^{*}\right), \varphi_{-s}\right\rangle=\left\langle\lambda^{*}\left(x^{*}\right)_{s}, \varphi\right\rangle .
\end{aligned}
$$

Hence a closely similar argument to that above yields $\left\|\lambda^{*}\right\|_{\left[X^{\bullet}, W C^{\prime}\right]}=\|\lambda\|_{W C^{\prime}(X)}$, and the equivalence of $W C^{r}$ and $C_{r}^{k}$ for scalar-valued functions implies 2(a).

All of the other equivalences of Lemma 4.5.2 proceed in the same way.

Note that since $\lambda_{y} \in L^{1}(X) \forall y>0$, the $M^{*}$ norm and the $M$ norm coincide on $\lambda_{\boldsymbol{y}}$

Lemma 4.5.3. For every positive $q>0$, let $J^{q}$ be the Bessel potential of order $q$; let $r>0$ and $k$ be an integer $k>r+q$. Then the operator $J^{q}$ defines an isomorphism of functors

$$
C_{r}^{k} \stackrel{J q}{\cong} C_{r}^{k+q}, \quad M_{r}^{q+k} \stackrel{J q}{\cong} M_{r+q}^{k}
$$


for every $r, q>0$.

Proof. This lemma is proved by the same method as Lemma 4.5.2 using the isometries between $\left[X^{*}, C_{r}^{k}\right]$ and $C_{r}^{k}(X)$ and $\left[X^{*}, M_{r}^{k}\right]$ and $M_{r}^{k}(X)$. It is only necessary to observe that for every $\varphi \in L^{1}$,

$$
\begin{aligned}
\left\langle\left(J^{q} \lambda\right)^{*}\left(x^{*}\right), \varphi\right\rangle & =\left\langle x^{*}, J^{q} \lambda(\varphi)\right\rangle=\left\langle x^{*}, \lambda\left(J^{q} \varphi\right)\right\rangle \\
& =\left\langle\lambda^{*}\left(x^{*}\right), J^{q} \varphi\right\rangle=\left\langle J^{q}\left(\lambda^{*}\left(x^{*}\right)\right), \varphi\right\rangle .
\end{aligned}
$$

A similar relationship holds for the functor $M_{r}^{k}$.

It is now possible to prove the theorem. Let $X, Y \in \mathcal{B}$ and let $J_{X \otimes Y}^{1}$ and $J_{Y}^{1}$ be the isomorphisms defined by (4.46) and Lemma 4.5 .3 for $q=1$ and the Banach spaces $X \otimes Y$ and $Y$. Define an operator $i_{Y}$ by

$$
i_{Y}(h)(f)=J_{X \otimes Y}^{-1}\left[J_{Y}^{1} h * f\right]
$$

for every $h \in M J(Y)$ and $f \in C^{\prime}(X)$, where * is the tensor convolution.

Lemma 4.5.4. Let $i_{Y}$ be the map defined by (4.47) for $X, Y \in \mathcal{B}$. Then $i_{Y}: M J(Y) \rightarrow \operatorname{Hom}_{\circledast}\left[C^{r}, C^{r}\right](Y)$ with norm independent of the Banach spaces $X$, $Y \in \mathcal{B}$.

Proof. I shall show that the mapping $\tau_{Y}$ defined by $\tau_{Y}(h)(f)=J_{Y}^{1} h * f$ maps $M J(Y) \otimes C^{r}(X)$ into $C_{1+r}^{3}(X \otimes Y)$ with a norm independent of $X, Y \in \mathcal{B}$. By Lemma 4.5.3, $J^{-1}$ defines a functor isomorphism $J^{-1}: C_{r}^{3} \rightarrow C_{r}^{1}$ and by Lemma 4.5.2, $C_{1}^{r}(X \otimes Y)$ is identical to $C^{r}(X \otimes Y)$ up to equivalence of norms independently of $X, Y \in \mathcal{B}$; hence the existence of a homomorphism $i_{Y}$ will have been proved.

Let $f \in C^{r}(X)$; therefore $f \in C_{r}^{\prime}(X)$ and hence

$$
\|f\|_{C \lambda(x)}=\sup _{y>0}\left\|f_{y}^{\prime} y^{1-r}\right\|_{L^{\infty}(x)}
$$

Similarly since $h \in M J(Y)$, it follows that

$$
\left\|J_{Y}^{1} h\right\|_{M_{1}^{2}(Y)}=\sup _{y>0}\left\|\left(J_{Y}^{1} h\right)_{y}^{(2)} y\right\|_{M(Y)} .
$$

Since, for every $y>0,\left(J_{Y}^{1} h\right)_{y}^{(2)} \in M(Y)$, and $f_{y}^{\prime} \in L^{\infty}(X)$, it follows by Theorem 4.4 that the tensor convolution of $\left(J_{Y}^{1} h\right)_{y}^{(2)}$ and $f_{y}^{\prime}$ is well defined. By the equalities (4.48) and (4.49) it follows that

$$
y^{2-r}\left(J^{1} h\right)_{y}^{(2)} * f_{y}^{\prime} \in L^{\infty}(X \otimes Y)
$$

Let $g_{2 y}=\left(J^{1} h\right)_{y} * f_{y}$. By Theorem 4.4, it follows that

$$
\begin{aligned}
\left\|g_{2 y}^{(3)} y^{2-r}\right\|_{L^{\infty}(X \otimes Y)} & =\left\|y^{2-r}\left(J_{Y}^{1} h\right)_{y}^{(2)} * f_{y}^{\prime}\right\|_{L^{\infty}(X \otimes Y)} \\
& \leq\left\|y\left(J_{Y}^{1} h\right)_{y}^{(2)}\right\|_{M(Y)} \cdot\left\|y^{1-r} f_{y}^{\prime}\right\|_{L^{\infty}(X)}
\end{aligned}
$$


and therefore by the Definitions (4.48) and (4.49)

$\|g\|_{C_{1+r}^{3}(X \otimes Y)} \leq 2^{2-r}\left\|J_{Y}^{1} h\right\|_{M_{1}^{2}(Y)}\|f\|_{C \gamma(X)}$.

Thus $\tau_{Y}(h)(f)=g$ and $\tau_{Y}: M_{1}^{2}(Y) \otimes C_{r}^{1}(Y) \rightarrow C_{1+r}^{3}(X \otimes Y)$ with the norm of $\tau_{Y}$ clearly independent of $X, Y \in \mathcal{B}$.

It is clear from the representation (4.47) of the map $i$ that $i$ is a monomorphism.

To show that the mapping $i_{Y}$ maps onto $\operatorname{Hom}_{\otimes}\left[C^{r}, C^{r}\right](Y)$, let $H$ $\in \operatorname{Hom}_{\otimes}\left[C^{r}, C^{\prime}\right](Y)$. Then $H_{C}: C^{\prime} \rightarrow C^{r}(Y)$. For every $\varphi \in C^{\prime}$, by Proposition 4.0 there exists a $Y$-valued distribution $H$ such that $H_{C} \varphi=H_{0} * \varphi$. Since $H_{\mathrm{C}} \varphi \in C^{r}(Y)$, it follows by Lemma 4.5.2 that

$$
J_{Y}^{1} H_{\mathrm{C}} \varphi=J_{Y}^{1} H_{0} * \varphi \in W C^{1+r}(Y)
$$

where by Lemma 4.5.3 the norm of $J_{Y}^{1}$ is independent of $Y \in \mathcal{B}$.

Let $\psi \in C$. By Lemma 4.5.3, $J^{r} \psi \in C^{r}$. Hence

$$
\left(J^{r} \psi\right) * J^{1} H_{0} \in W C^{1+r}(Y)
$$

By Lemma 4.5.3, it follows that

$$
J_{Y}^{-r}\left(J^{r} \psi\right) * J^{1} H_{0}=\psi * J^{1} H_{0} \in W C^{1}(Y)
$$

Therefore by Lemma 4.5.2, $\left\|\psi *\left(J^{1} H_{0}\right)_{y}^{(2)} y\right\|_{L^{\infty}(Y)}<\infty$. Thus

$$
H \in \operatorname{Hom}_{\otimes}\left[C^{r}, C^{r}\right](Y)
$$

defines an element of $\operatorname{Hom}_{\circledast}\left[L^{\infty}, L^{\infty}\right](Y)$ by the mapping

$$
H \rightarrow\left(J^{1} H_{\mathrm{C}}\right)_{y}^{(2)} y \text {. }
$$

It is clear from (4.53), (4.54) and (4.55) above that the mapping (4.56) has norm independent of the Banach space $Y$. By Theorem 4.4, $\left(J^{1} H_{C}^{(2)}\right)_{y} y \in M(Y)$ and therefore $H_{0} \in M J(Y)$.

To show that the diagram

$$
\begin{gathered}
M J(Y) \stackrel{i_{Y}}{\longrightarrow} \operatorname{Hom}_{\otimes}\left[C^{r}, C^{r}\right](Y) \\
M J(\alpha) \mid \\
M J(Z) \stackrel{i_{Z}}{\longrightarrow} \text { Hom }_{\otimes}\left[C^{r}, C^{r}\right](Z)
\end{gathered}
$$

is commutative, let $X, Y, Z \in \mathcal{B}, h \in M J(Y)$, and $f \in C^{r}(X)$. Then $J_{Y}^{1} h$ $\in W M^{1}(Y)$ and 


$$
\begin{aligned}
i_{Z} \circ M J(\alpha)(h)(f) & =i_{Z}(\alpha \circ h)(f)=(\alpha \circ h) * f \\
& =J_{Z \otimes X}^{-1}\left(J_{Z}^{1}(\alpha h) * f\right)=J_{Z \otimes X}^{-1} C^{\prime}\left(\alpha \otimes 1_{X}\right) \int\left(J_{Y}^{1} h\right) * f \\
& =\operatorname{Hom}_{\otimes}\left[C^{r}, C^{r}\right](\alpha) i_{Y}(h)(f)
\end{aligned}
$$

the theorem is proved.

As in the case of Theorem 4.4 various corollaries of interest to applications follow from Theorem 4.5 by the use of the properties of the mappings $\Lambda$ and $\tau$.

Corollary 4.5.1. Let $T^{n}$ be a toroidal group, $M_{0}^{\prime}$ the category of normal Banach modules in $\mathcal{B}_{0}$, on $T^{n}$, and $\mathcal{B}$ the category of Banach spaces. Let $C^{r}$ be the Lipschitz functor for $0<r<1$ acting on $M_{0}^{\prime}$. Then there exists a monomorphic embedding

$$
M J \subseteq \mathrm{Hom}_{\otimes}\left[\Lambda C^{r}, \Lambda C^{r}\right]
$$

where the functors in (4.57) act on the category $\mathcal{B}$.

Proof. This corollary follows immediately from the properties of $\Lambda$ and $\tau$ given by Theorem 2.3 .

Corollary 4.5.1 may be applied to obtain a much more particular result for another class of operators prominent in applications.

Let $X$ be a Banach module of functions on a toroidal group $T^{n}$. A convolution operator $H$ is termed a singular integral operator if it is of the form

$$
\overline{H f}(s)=\int_{E^{n}} \frac{\Omega(s-t)}{|s-t|^{n}} \tilde{f}(t) d t ;
$$

where $\Sigma_{n}$ is the surface of the sphere $|x|=1$ in $E^{n}, \int_{\Sigma_{n}} \Omega(\theta) d \theta=0$, and $\bar{f}$ denotes the periodic extension of $f$ to $E^{n}$.

Corollary 4.5.2. Let $T^{n}$ be a toroidal group and $X \in \mathcal{B}_{0}$. Let $C^{r}$ be the Lipschitz functor for $0<r<1$. Then $M J \subseteq\left[\Lambda C^{r}(X), \Lambda C^{r}(X)\right]$.

Proof. It is very well known from the results of Giraud (see [23]) that if $H$ is a singular integral operator then $H: C^{r} \rightarrow C^{r}$, where $C^{r}$ is the Lipschitz space $C^{r}(\mathrm{C})$ of continuous functions. By Theorem 4.5 for $X=Y=\mathrm{C}, H \in M J$. Therefore it follows at once by Corollary 4.5.1 that $H: \Lambda C^{r}(X) \rightarrow \Lambda C^{r}(X)$ for any Banach space $X \in \mathcal{B}_{0}$ and singular integral operator $H$.

The next result leads to some important commutator results (see [4]).

Corollary 4.5.3. Let $T^{n}$ be a toroidal group and $X$ a Banach module over $M_{0}$ and over $L^{\infty}$. Let $0<r<1$ and suppose that $c$ is a function of class $W C^{1+r}$. Define an operator $H(c, f)$ by

$$
H(c, f)(s)=\int \frac{c(s)-c(t)}{|s-t|^{n+1}} \bar{f}(t) d t
$$


where $\bar{f}$ is the periodic extension of $f \in X$ to $E^{n}$. Then

$$
H: W C^{1+r} \otimes \Lambda C^{r}(X) \rightarrow \Lambda C^{\prime}(X) .
$$

Proof. This corollary follows easily from Corollary 4.5.1 and Corollary 4.4.5 after the observation that by Taylor's theorem

$$
c(s)-c(t)=\sum_{i=1}^{n}\left(s_{i}-t_{i}\right) \frac{\partial c}{\partial x_{i}}(s)+R_{0}(s, t)|s-t|^{1+r}
$$

where $R_{0}(s, t)$ is continuous on $T^{n} \times T^{n}$ and $\partial c / \partial x_{i} \in C^{r}$ for every $i$.

Remarks. One particular case of Corollary 4.4.1, the case in which $X=L$, was proved by Taibleson in his paper [42]. Singular integral operators of various types with vector-valued kernels, and acting on $L^{P}$ spaces of vector-valued functions, have been considered by J. Schwartz [34], and Benedek, Calderón, and Panzone in [1].

Continuity of operators of the form (4.54) given in Corollary 4.5.3 implies. commutator results of a type very well known in the theory of algebras of singular integral operators or pseudo-differential operators. For the particular spaces $L^{p}$ and $L^{2}$ these algebras have been extensively studied (cf. [4] or [16]).

Of possible generalizations and extensions of these results, it is clear from the arguments of Theorem 4.5 that they may be generalized to the context of spaces formed from $X \in \mathcal{B}$ on analogy with the spaces $L p, \lambda$ which have been the subject of recent articles [27], [28] by Peetre.

Singular integral operators of the types (4.53) or (4.54) have been very extensively studied for their applications to partial differential equations. By composition with other convolution operators similar to the Bessel potentials, it has been possible to construct inverses to partial differential operators; using results on continuity of singular integral operators from spaces $L^{P}$ to $L^{\prime}$ or $C^{r}$ to $C^{r}$ many authors ([23], [27], [37] and others) have obtained estimates and existence theorems for the solutions of elliptic partial differential operators both for Sobolev spaces $W^{m} L^{p}$ and various fractional Sobolev spaces of the types discussed by Taibleson [41]. As commented above, the spaces $\Lambda C^{r}(X)$ for $0<r<1$ form a fractionalization similar to that of Taibleson's spaces $\Lambda(\alpha, p, \infty)$ for general Banach modules $X$ in place of the $L^{p}$ spaces; Corollaries 4.5.1 and 4.5.3 suggest the possibility of extending these theorems on estimates and solvability to a large class of Banach function spaces.

\section{REFERENCES}

1. A. Benedek, A. P. Calderón and R. Panzone, Convolution operators on Banach space valued functions, Proc. Nat. Acad. Sci. U.S.A. 48 (1962), 356-365. MR 24 \# A3479.

2. R. G. Bartle, A general bilinear vector integral, Studia Math. 15 (1956), 337-352. MR 18, 289.

3. A. P. Calderón, Intermediate spaces and interpolation, the complex method, Studia Math. 24 (1964), 113-190. MR 29 \#5097. 
4. A. P. Calderón and A. Zygmund, Singular integral operators and differential equations, Amer. J. Math. 79 (1957), 901-921. MR 20 \#7196.

5. C. V. Comisky, Multipliers of Banach modules, Nederl. Akad. Wetensch. Proc. Ser. A $74=$ Indag. Math. 33 (1971), 32-38. MR 43 \#5307.

6. C. Corduneanu, Admissibility with respect to an integral operator and applications, Studies in Appl. Math., no. 5, SIAM, Philadelphia, Pa., 1969, pp. 55-64.

7. T. K. Donaldson, Singular integrals in Banach function spaces, Research Report, Australian National University, 1971.

8. J. Dieudonné, Sur les espaces de Köthe, J. Analyse Math. 1 (1951), 81-115. MR 12, 834.

9. N. Dinculeanu, Vector measures, Internat. Series of Monographs in Pure and Appl. Math., vol. 95, Pergamon Press, Oxford; VEB Deutscher Verlag der Wissenschaften, Berlin, 1967. MR 34 \#6011b.

10. N. Dunford and J. T. Schwartz, Linear operators. I: General theory, Pure and Appl. Math., vol. 7, Interscience, New York, 1958. MR 22 \#3302.

11. R. E. Edwards, Fourier series: A modern introduction. Vols. I, II, Holt, Rinehart and Winston, New York, 1967. MR 35 \# 7062; MR 36 \#5588.

12. A. Figa-Talamanca and G. I. Gaudry, Density and representation theorems for multipliers of the type (p, q), J. Austral. Math. Soc. 7 (1967), 1-6. MR 35 \#666.

13. A. Grothendieck, Produits tensoriels topologiques et espaces nucléaires, Mem. Amer. Math. Soc. No. 16 (1955). MR 17 \#763.

14. L. Hörmander, Estimates for translation invariant operators in $L^{p}$ spaces, Acta Math. 104 (1960), 93-140. MR 22 \# 12389.

15. Y. Katznelson, An introduction to harmonic analysis, Wiley, New York, 1968. MR 40 \# 1734.

16. J. J. Kohn and L. Nirenberg, An algebra of pseudodifferential operators, Comm. Pure Appl. Math. 18 (1965), 269-305. MR 31 \#636.

17. M. A. Krasnosel'skil and Ja. B. Rutickil, Convex functions and Orlicz spaces, Problems of Contemporary Math., GITTL, Moscow, 1958; English transl., Noordhoff, Groningen, 1961. MR 21 \#5144; MR 23 \#A4016.

18. G. G. Lorentz, Bernstein polynomials, Mathematical Expositions, no. 8, Univ. of Toronto Press, Toronto, 1953. MR 15, 217.

19._, Some new functional spaces, Ann. of Math. (2) 51 (1950), 37-55. MR 11, 442.

20.—, On the theory of spaces $\Lambda$, Pacific J. Math. 1 (1951), 411-429. MR 13, 470.

21. W. A. J. Luxemburg, Banach function spaces, Thesis, Technische Hogeschool te Delft, 1955. MR 17, \#285.

22. J. L. Massera and J. J. Schaeffer, Linear differential equations and function spaces, Pure and Appl. Math., vol. 21, Academic Press, New York, 1966. MR 35 \#3197.

23. S. G. Mihlin, Multi-dimensional singular integrals and integral equations, Fizmatgiz, Moscow, 1962; English transl., Pergamon Press, New York, 1965. MR 27 \#5105; MR 32 \#2866.

24. B. S. Mitjagin and A. S. Švarc, Functors in categories of Banach spaces, Uspehi Mat. Nauk 19 (1964), no. 2 (116), 65-130 = Russian Math. Surveys 19 (1964), no. 2, 65-127. MR 29 \#3866.

25. R. O'Neil, Fractional integration in Orlicz spaces. I, Trans. Amer. Math. Soc. 115 (1965), 300-328. MR 33 \#3087.

26. - Integral transforms and tensor products on Orlicz spaces and $L(p, q)$ spaces, J. Analyse Math. 21 (1968), 1-276.

27. J. Peetre, On the theory of $\mathcal{L}_{p, \lambda}$ spaces, J. Functional Analysis 4 (1969), 71-87. MR 39 \#3300.

28. - On convolution operators leaving $L^{p, \lambda}$ spaces invariant, Ann. Mat. Pura Appl. (4) 72 (1966), 295-304. MR 35 \#12.

29. W. Pogorzelski, Integral equations and their applications. Vols. 1-3, PWN, Warsaw, 1953, 1958, 1960; English transl., Pergamon Press, Oxford; PWN, Warsaw, 1966. MR 16, 46; MR 20 \#213; MR 28 \#2415; MR 34 \# 1811. 
30. M. A. Rieffel, Induced Banach representations of Banach algebras and locally compact groups, J. Functional Analysis 1 (1967), 443-491.

31. - Multipliers and tensor products of $L^{p}$ spaces of locally compact groups, Studia Math. 33 (1969), 71-82. MR 39 \#6078.

32. J. J. Schäffer, Function spaces with translations, Math. Ann. 137 (1959), 209-262. MR 21 \#287.

33. R. Schatten, $A$ theory of Cross spaces, Ann. of Math. Studies, no. 26, Princeton Univ. Press, Princeton, N.J., 1950. MR 12, 186.

34. J. T. Schwartz, A remark on inequalities of Calderón-Zygmund type for vector-valued functions, Comm. Pure Appl. Math. 14 (1961), 785-799. MR 26 \#597.

35. L. Schwartz, Théorie des distributions. Tome I, Actualités Sci. Indust., no. 1091, Hermann, Paris, 1950. MR 12, 31.

36.—_, Théorie des distributions d̀ valeurs vectorielles. I, Ann. Inst. Fourier (Grenoble) 7 (1957), 1-141. MR 21 \#6534.

37. R. T. Seeley, Singular integrals and boundary value problems, Amer. J. Math. 88 (1966), 781-809. MR 35 \#810.

38. H. Shapiro, A Tauberian theorem related to approximation theory, Acta Math. 120 (1968), 279-292. MR 39 \#689.

39. V. L. Shapiro, Fourier series in several variables, Bull. Amer. Math. Soc. 70 (1964), 48-93. MR 28 \# 1448.

40. A. S. Svarc, Duality of functors, Dokl. Akad. Nauk SSSR 148 (1963), 288-291 = Soviet Math. Dokl. 4 (1963), 89-92. MR 29 \#5887.

41. M. S. Taibleson, On the theory of Lipschitz spaces of distributions on Euclidean n-space. I. Principal properties, J. Math. Mech. 13 (1964), 407-479. MR 29 \#462.

42.—, On the theory of Lipschitz spaces of distributions on Euclidean n-space. II. Translation invariant operators, duality and interpolation, J. Math. Mech. 14 (1965), 821-839. MR 31 \#5087.

43. A. Zygmund, Smooth functions, Duke Math J. 12 (1945), 47-76. MR 7, 60.

44. - On the preservation of classes of functions, J. Math. Mech. 8 (1959), 889-895; erratum, ibid. 9 (1960), 663. MR 22 \#8277.

45.—, Trigonometric series. Vols. I, II, 2nd ed., Cambridge Univ. Press, New York, 1959. MR 21 \#6498.

Department of Mathematics SGS, Australian National University, Canberra, Australu 